Supporting Information

\title{
Light-Mediated Asymmetric Aliphatic C-H Alkylation with Hydrogen Atom Transfer Catalyst and Chiral Phosphoric Acid
}

\author{
Zhen-Yao Dai ${ }^{\dagger}$, Zhong-Sheng Nong ${ }^{\dagger}$, Pu-Sheng Wang*† \\ ${ }^{\dagger}$ Department of Chemistry, University of Science and Technology of China, Hefei, 230026, China \\ E-mail:pusher@ustc.edu.cn
}

\section{Table of contents}

1 General Data .2

2 Experimental Procedures . .2

3 Optimization of Reaction Conditions . .3

4 Control Experiment. .5

5 Characterization Data for the Products . .5 


\section{General Data}

NMR spectra were recorded on a Brucker- $400 \mathrm{MHz}$ spectrometer and a Brucker-500 MHz spectrometer. Mass spectra were recorded on a Thermo LTQ Orbitrap XL (ESI+) or a P-SIMS-Gly of Brucker Daltonics Inc (EI+). Infrared spectra were recorded on a Nicolet MX-1E FT-IR spectrometer. HPLC analysis was performed on Waters-Breeze (2487 Dual Absorbance Detector and 1525 Binary HPLC Pump) and Agilent 1200 (UV detection monitored at $254 \mathrm{~nm}$ ). Chiralpak AS-H, AD-H, OJ-H, IB, IC, ID, IE, IF, IG columns were purchased from Daicel Chemical Industries, LTD. Optical rotations were measured on Perkin Elmer Model 343 Polarimeter. Starting materials and solvents were purchased from commercial suppliers (Aldrich, Alfa, TCI, Adamas-beta) and used as supplied unless otherwise stated.

\section{Experimental Procedures}

Starting materials were purchased from commercial suppliers (Aldrich, Acros, TCI, Adamas-data, Energy etc.) and used as supplied unless otherwise stated. Chiral spiro-phosphoric acids were known compounds and prepared according to previously reported procedures ${ }^{1}$. TBADB (tetrabutylammonium decatungstate) was prepared by following the literature report ${ }^{2}$. Exocyclic-enones were prepared by following the literature report ${ }^{3}$. All solvents were purified and dried according to standard methods prior to use, unless stated otherwise.

\section{General Experimental Procedures for Exocyclic-enones and Cyclohexane}

To a Ar-purged $10 \mathrm{~mL}$ schlenk tube were added exocyclic enones $1(0.1 \mathrm{mmol})$, Cyclohexane $(2 \mathrm{~mL})$, Chiral spiro-phosphoric acid A4 (2 mol\%), TBADT (4 mol\%), solvent of PhCN (3.0 mL) and a stir bar. Afterwards, the reaction mixture was degassed by three freeze pump-thaw cycles. The schlenk tube was refilled with nitrogen. After being stirred for 3 hours at $30{ }^{\circ} \mathrm{C}$ under $390 \mathrm{~nm}$ LEDs, the solvent was removed under vacuum and the residue was purified by flash chromatography $\left(\mathrm{SiO}_{2}\right.$, ethyl acetate / petroleum ether $\left.=10: 1\right)$ to provide product 3-21.

\section{General Experimental Procedures for Exocyclic-enones and other Alkanes or Alkenes}

To a Ar-purged $10 \mathrm{~mL}$ schlenk tube were added exocyclic enones $1(0.1 \mathrm{mmol})$, R-H 2 (2 mmol), Chiral spiro-phosphoric acid A4 (2 mol\%), TBADT (4 mol\%), solvent of MeCN: DCM =2: $1(0.5 \mathrm{~mL})$ and a stir bar. Afterwards, the reaction mixture was degassed by three freeze pump-thaw cycles. The schlenk tube was refilled with nitrogen. After being stirred for 3 hours at $30{ }^{\circ} \mathrm{C}$ under $390 \mathrm{~nm}$ LEDs, the solvent was removed under vacuum and the residue was purified by flash chromatography $\left(\mathrm{SiO}_{2}\right.$, ethyl acetate / petroleum ether $\left.=10: 1\right)$ to provide product 22-24,32,33.

\section{General Experimental Procedures for Exocyclic-enones and Toluene}

To a Ar-purged $10 \mathrm{~mL}$ schlenk tube were added exocyclic enones $1(0.1 \mathrm{mmol})$, toluene $2(2 \mathrm{mmol})$, Chiral spiro-phosphoric acid A4 (2 mol\%), TBADT (4 mol\%), PhCN (0.25 mL) and a stir bar. Afterwards, the reaction mixture was degassed by three freeze pump-thaw cycles. The schlenk tube was refilled with nitrogen. After being stirred for 20 hours at $30{ }^{\circ} \mathrm{C}$ under $390 \mathrm{~nm}$ LEDs, the solvent was removed under vacuum and the residue was purified by flash chromatography $\left(\mathrm{SiO}_{2}\right.$, ethyl acetate / petroleum ether $\left.=10: 1\right)$ to provide product 25-31. 

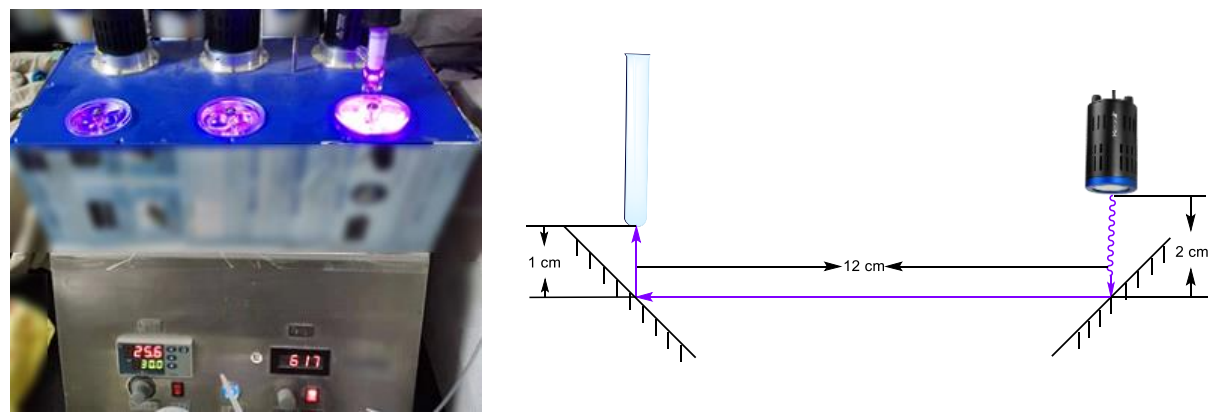

The reaction mixture is irradiated in a photoreactor with two mirrors, wich reflect the light emitted by 34 Kessil PR160-390 lamps, $15 \mathrm{~cm}$ away. The reactor, to maintain the temperature, has cooling system itself.

\section{Optimization of Reaction Conditions}

\section{Table S1. Optimization of Reaction Conditions for Exocyclic-enones and Cyclohexane ${ }^{a}$}

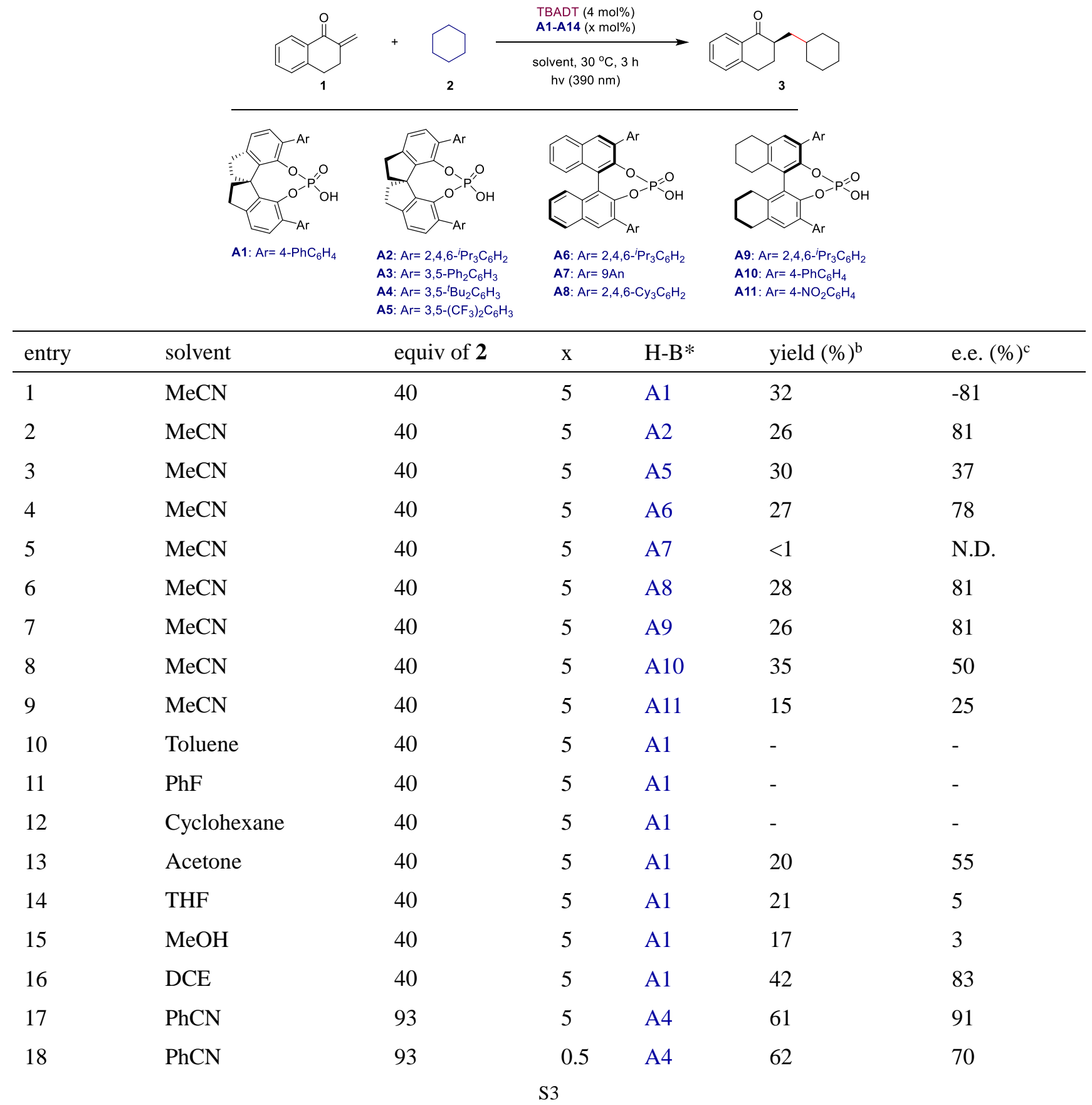


${ }^{a}$ Reaction conditions: $1(0.10 \mathrm{mmol})$, cyclohexane 2 (4.0 mmol), H-B* (0.5-5 mol\%), TBADT (4 mol\%), solvent $(1.0 \mathrm{ml}), 30{ }^{\circ} \mathrm{C}, 40 \mathrm{~W}$ LEDs $\left(\lambda_{\max }=390 \mathrm{~nm}\right), 3 \mathrm{~h}$, under nitrogen. ${ }^{b}$ Determined by ${ }^{1} \mathbf{H}$ NMR analysis using trimethyl 1,3,5-benzenetricarboxylate as an internal standard. ${ }^{c}$ Determined by chiral-phase HPLC analysis. The absolute stereochemistry was assigned by analogy.

Table S2. Optimization of Reaction Conditions for Exocyclic-enones and Cyclooctane ${ }^{a}$

\begin{tabular}{llllll} 
& equiv of 2 & yield $(\%)^{\mathrm{b}}$ & e.e. $(\%)^{\mathrm{c}}$ \\
\hline entry & solvent & equiv of solvent & 40 & 80 \\
1 & $\mathrm{PhCN}$ & $1 \mathrm{~mL}$ & 40 & 15 & 80 \\
2 & $\mathrm{MeCN}$ & $1 \mathrm{~mL}$ & 40 & - & - \\
3 & $\mathrm{DCM}$ & $1 \mathrm{~mL}$ & 40 & 60 & 85 \\
4 & $\mathrm{MeCN}: \mathrm{DCM}=3: 1$ & $1 \mathrm{~mL}$ & 40 & 69 & 86 \\
5 & $\mathrm{MeCN}: \mathrm{DCM}=2: 1$ & $1 \mathrm{~mL}$ & 40 & 77 & 89 \\
6 & $\mathrm{MeCN}: \mathrm{DCM}=2: 1$ & $1 \mathrm{~mL}$ & 20 & 79 & 87 \\
7 & $\mathrm{MeCN}: \mathrm{DCM}=2: 1$ & $2 \mathrm{~mL}$ & 20 & 73 & 92 \\
8 & $\mathrm{MeCN}: \mathrm{DCM}=2: 1$ & $0.5 \mathrm{~mL}$ & 20 & $\mathrm{~mol} \%)$
\end{tabular}

${ }^{a}$ Reaction conditions: 1 ( $\left.0.10 \mathrm{mmol}\right)$, cyclohexane 2 (4.0 mmol), H-B* (2 mol\%), TBADT (4 mol\%), solvent (1.0 $\mathrm{ml}), 30{ }^{\circ} \mathrm{C}, 40 \mathrm{~W}$ LEDs $\left(\lambda_{\max }=390 \mathrm{~nm}\right), 3 \mathrm{~h}$, under nitrogen. ${ }^{b}$ Determined by ${ }^{1} \mathbf{H}$ NMR analysis using trimethyl 1,3,5-benzenetricarboxylate as an internal standard. ${ }^{c}$ Determined by chiral-phase HPLC analysis. The absolute stereochemistry was assigned by analogy.

Table S3. Optimization of Reaction Conditions for Exocyclic-enones and Toluene ${ }^{a}$

\begin{tabular}{|c|c|c|c|c|c|}
\hline entry & solvent & equiv of solvent & equiv of 2 & yield $(\%)^{b}$ & e.e. $(\%)^{c}$ \\
\hline 1 & $\mathrm{MeCN}: \mathrm{DCM}=2: 1$ & $0.5 \mathrm{~mL}$ & 20 & 25 & 80 \\
\hline 2 & DCM & $0.5 \mathrm{~mL}$ & 20 & 14 & 88 \\
\hline 3 & $\mathrm{MeCN}$ & $0.5 \mathrm{~mL}$ & 20 & 37 & 83 \\
\hline 4 & $\mathrm{PhCN}$ & $0.5 \mathrm{~mL}$ & 20 & 45 & 86 \\
\hline 5 & $\mathrm{PhCN}$ & $0.5 \mathrm{~mL}$ & 10 & 21 & 85 \\
\hline 6 & $\mathrm{PhCN}$ & $0.5 \mathrm{~mL}$ & 40 & 45 & 86 \\
\hline 7 & $\mathrm{PhCN}$ & $0.1 \mathrm{~mL}$ & 20 & 42 & 83 \\
\hline 8 & $\mathrm{PhCN}$ & $0.25 \mathrm{~mL}$ & 20 & 58 & 85 \\
\hline 9 & $\mathrm{PhCN}$ & $1 \mathrm{~mL}$ & 20 & 34 & 84 \\
\hline 10 & $\mathrm{PhCN}$ & $2 \mathrm{~mL}$ & 20 & 37 & 84 \\
\hline
\end{tabular}

${ }^{a}$ Reaction conditions: 1 ( $\left.0.10 \mathrm{mmol}\right)$, cyclohexane 2 (4.0 mmol), H-B* (2 mol\%), TBADT (2 mol\%), solvent (1.0 $\mathrm{ml}), 30{ }^{\circ} \mathrm{C}, 40 \mathrm{~W}$ LEDs $\left(\lambda_{\max }=390 \mathrm{~nm}\right), 3 \mathrm{~h}$, under nitrogen. ${ }^{b}$ Determined by ${ }^{1} \mathbf{H}$ NMR analysis using trimethyl 1,3,5-benzenetricarboxylate as an internal standard. ${ }^{c}$ Determined by chiral-phase HPLC analysis. The absolute stereochemistry was assigned by analogy. 


\section{Control Experiment}

To a Ar-purged $10 \mathrm{~mL}$ schlenk tube were added racemic 2-(cyclohexylmethyl)-3,4-dihydronaphthalen-1(2H)-one 3 (0.1 mmol), Cyclohexane (2 mL), Chiral spiro-phosphoric acid A4 (2 mol\%), TBADT (4 mol\%), solvent of $\mathrm{PhCN}(3.0 \mathrm{~mL})$ and a stir bar. Afterwards, the reaction mixture was degassed by three freeze pump-thaw cycles. The schlenk tube was refilled with nitrogen. After being stirred for 3 hours at $30{ }^{\circ} \mathrm{C}$ under $390 \mathrm{~nm}$ LEDs, the solvent was removed under vacuum and the residue was purified by flash chromatography $\left(\mathrm{SiO}_{2}\right.$, ethyl acetate $/$ petroleum ether $=10: 1)$.

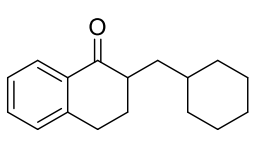

racemic

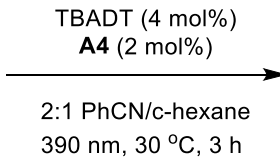

$390 \mathrm{~nm}, 30^{\circ} \mathrm{C}, 3 \mathrm{~h}$

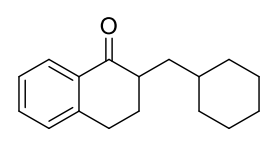

$2 \%$ e.e.

\section{Characterization Data for the Products}

(R)-2-(cyclohexylmethyl)-3,4-dihydronaphthalen-1(2H)-one (3) Colorless oil. Yield: 83\%. Enantiomeric excess: 92\%, determined by HPLC (CHIRALPAK IE, hexane/ isopropanol = 94/6, flow rate $1.0 \mathrm{~mL} / \mathrm{min}, \mathrm{T}=$ $30^{\circ} \mathrm{C}, 254 \mathrm{~nm}$ ): $\mathrm{t}_{\mathrm{R}}=6.565 \mathrm{~min}$ (minor), $\mathrm{t}_{\mathrm{R}}=6.899 \min$ (major). $[\alpha]_{\mathrm{D}^{20}}=-1.25$ (c 1.5, $\left.\mathrm{CHCl}_{3}\right) .{ }^{1} \mathbf{H} \mathbf{~ N M R}(400 \mathrm{MHz}$, Chloroform- $d$ ) $\delta 8.02(\mathrm{dd}, J=7.9,1.4 \mathrm{~Hz}, 1 \mathrm{H}), 7.44(\mathrm{td}, J=7.5,1.5 \mathrm{~Hz}, 1 \mathrm{H}), 7.29(\mathrm{t}, J=7.5 \mathrm{~Hz}, 1 \mathrm{H}), 7.22(\mathrm{~d}, J=$ $7.6 \mathrm{~Hz}, 1 \mathrm{H}), 3.06-2.88(\mathrm{~m}, 2 \mathrm{H}), 2.58(\mathrm{ddd}, J=10.4,5.2,3.4 \mathrm{~Hz}, 1 \mathrm{H}), 2.28-2.16(\mathrm{~m}, 1 \mathrm{H}), 1.93-1.79(\mathrm{~m}, 2 \mathrm{H})$, $1.79-1.61(\mathrm{~m}, 5 \mathrm{H}), 1.47-1.37(\mathrm{~m}, 1 \mathrm{H}), 1.34-1.11(\mathrm{~m}, 4 \mathrm{H}), 1.05-0.80(\mathrm{~m}, 2 \mathrm{H}) .{ }^{13} \mathbf{C} \mathbf{~ N M R}\left(101 \mathrm{MHz}, \mathrm{CDCl}_{3}\right)$ $\delta 201.12,143.97,133.14,132.65,128.74,127.56,126.62,44.70,36.99,34.96,34.24,32.71,28.51,28.28,26.74$, 26.53, 26.39. HRMS (ESI) $\mathrm{m} / \mathrm{z}(\mathrm{M}+\mathrm{H})^{+}$calculated for $\mathrm{C}_{17} \mathrm{H}_{23} \mathrm{O}: 243.1749$, observed: 243.1739. The absolute configuration was tentatively assigned by analogy.

(R)-2-(cyclohexylmethyl)-7-methyl-3,4-dihydronaphthalen-1(2H)-one (4) Colorless oil. Yield: 80\%. Enantiomeric excess: $86 \%$, determined by HPLC (CHIRALPAK IF, hexane/ isopropanol $=97 / 3$, flow rate 1.0 $\mathrm{mL} / \mathrm{min}, \mathrm{T}=30^{\circ} \mathrm{C}, 254 \mathrm{~nm}$ ): $\mathrm{t}_{\mathrm{R}}=5.986 \min \left(\right.$ minor), $\mathrm{t}_{\mathrm{R}}=6.665 \min$ (major). $[\alpha]_{\mathrm{D}}{ }^{20}=-0.96\left(\mathrm{c} 0.64, \mathrm{CHCl}_{3}\right) .{ }^{1} \mathbf{H}$ NMR $(400 \mathrm{MHz}$, Chloroform- $d$ ) $\delta 7.82(\mathrm{~d}, J=1.9 \mathrm{~Hz}, 1 \mathrm{H}), 7.27(\mathrm{dd}, J=7.2,2.2 \mathrm{~Hz}, 1 \mathrm{H}), 7.12(\mathrm{~d}, J=7.7 \mathrm{~Hz}$, $1 \mathrm{H}), 3.04-2.83(\mathrm{~m}, 2 \mathrm{H}), 2.62-2.50(\mathrm{~m}, 1 \mathrm{H}), 2.35(\mathrm{~s}, 3 \mathrm{H}), 2.26-2.11(\mathrm{~m}, 1 \mathrm{H}), 1.91-1.76(\mathrm{~m}, 2 \mathrm{H}), 1.77-1.59$ $(\mathrm{m}, 5 \mathrm{H}), 1.45-1.36(\mathrm{~m}, 1 \mathrm{H}), 1.29-1.13(\mathrm{~m}, 4 \mathrm{H}), 1.04-0.80(\mathrm{~m}, 2 \mathrm{H}) .{ }^{13} \mathbf{C} \mathbf{N M R}\left(101 \mathrm{MHz}, \mathrm{CDCl}_{3}\right) \delta 201.44$, 141.09, 136.25, 134.13, 132.40, 128.67, 127.66, 44.70, 36.98, 34.96, 34.25, 32.70, 28.56, 27.80, 26.76, 26.54, 26.40, 21.10. HRMS (ESI) $\mathrm{m} / \mathrm{z}(\mathrm{M}+\mathrm{H})^{+}$calculated for $\mathrm{C}_{18} \mathrm{H}_{25} \mathrm{O}$ : 257.1905, observed: 257.1895. The absolute configuration was tentatively assigned by analogy.

(R)-2-(cyclohexylmethyl)-7-methoxy-3,4-dihydronaphthalen-1(2H)-one (5). Colorless oil. Yield: 79\%. Enantiomeric excess: 84\%, determined by HPLC (CHIRALPAK IB, hexane/ isopropanol $=99 / 1$, flow rate 1.0 $\mathrm{mL} / \mathrm{min}, \mathrm{T}=30^{\circ} \mathrm{C}, 254 \mathrm{~nm}$ ): $\mathrm{t}_{\mathrm{R}}=4.615 \min$ (minor), $\mathrm{t}_{\mathrm{R}}=4.991 \mathrm{~min}$ (major). $[\alpha]_{\mathrm{D}}{ }^{20}=5.4\left(\mathrm{c} 0.70, \mathrm{CHCl}_{3}\right) .{ }^{1} \mathbf{H ~ N M R}$ $(400 \mathrm{MHz}$, Chloroform- $d$ ) $\delta 7.50(\mathrm{~d}, J=2.8 \mathrm{~Hz}, 1 \mathrm{H}), 7.13(\mathrm{~d}, J=8.4 \mathrm{~Hz}, 1 \mathrm{H}), 7.03(\mathrm{dd}, J=8.4,2.8 \mathrm{~Hz}, 1 \mathrm{H}), 3.82$ (s, 3H), $3.00-2.81(\mathrm{~m}, 2 \mathrm{H}), 2.62-2.50(\mathrm{~m}, 1 \mathrm{H}), 2.26-2.11(\mathrm{~m}, 1 \mathrm{H}), 1.89-1.78(\mathrm{~m}, 2 \mathrm{H}), 1.77-1.62(\mathrm{~m}, 5 \mathrm{H})$, $1.46-1.35(\mathrm{~m}, 1 \mathrm{H}), 1.29-1.14(\mathrm{~m}, 4 \mathrm{H}), 1.03-0.82(\mathrm{~m}, 2 \mathrm{H}) .{ }^{13} \mathbf{C}$ NMR $\left(101 \mathrm{MHz}, \mathrm{CDCl}_{3}\right) \delta$ 201.12, 158.34, 136.58, 133.39, 129.96, 121.54, 109.44, 55.59, 44.51, 37.01, 34.99, 34.23, 32.72, 28.69, 27.40, 26.75, 26.53, 26.40. HRMS (ESI) $\mathrm{m} / \mathrm{z}(\mathrm{M}+\mathrm{H})^{+}$calculated for $\mathrm{C}_{16} \mathrm{H}_{26} \mathrm{O}_{2} \mathrm{Na}$ : 278.1855, observed: 273.1846. The absolute configuration was tentatively assigned by analogy. 
(R)-2-(cyclohexylmethyl)-7-hydroxy-3,4-dihydronaphthalen-1(2H)-one (6). White Solid. Yield: 60\%. Enantiomeric excess: 84\%, determined by HPLC (CHIRALPAK IF, hexane/ isopropanol $=97 / 3$, flow rate 1.0 $\mathrm{mL} / \mathrm{min}, \mathrm{T}=30^{\circ} \mathrm{C}, 254 \mathrm{~nm}$ ): $\mathrm{t}_{\mathrm{R}}=17.343 \mathrm{~min}$ (minor), $\mathrm{t}_{\mathrm{R}}=16.005 \mathrm{~min}$ (major). $[\alpha]_{\mathrm{D}}{ }^{20}=5.6\left(\mathrm{c} 1.2, \mathrm{CHCl}_{3}\right) .{ }^{1} \mathbf{H}$ NMR (400 MHz, Chloroform- $d$ ) $\delta 7.72(\mathrm{~d}, J=2.8 \mathrm{~Hz}, 1 \mathrm{H}), 7.13(\mathrm{~d}, J=8.3 \mathrm{~Hz}, 1 \mathrm{H}), 7.05(\mathrm{dd}, J=8.3,2.7 \mathrm{~Hz}$, 1H), $6.69(\mathrm{~s}, 1 \mathrm{H}), 2.99-2.82(\mathrm{~m}, 2 \mathrm{H}), 2.57$ (ddt, $J=10.0,8.3,4.8 \mathrm{~Hz}, 1 \mathrm{H}), 2.21(\mathrm{dq}, J=13.4,4.9 \mathrm{~Hz}, 1 \mathrm{H}), 1.89$ $-1.79(\mathrm{~m}, 2 \mathrm{H}), 1.76-1.62(\mathrm{~m}, 5 \mathrm{H}), 1.40(\mathrm{ddq}, J=11.2,5.8,2.8 \mathrm{~Hz}, 1 \mathrm{H}), 1.32-1.13(\mathrm{~m}, 4 \mathrm{H}), 1.02-0.81(\mathrm{~m}$, 2H). ${ }^{13}$ C NMR $\left(101 \mathrm{MHz}, \mathrm{CDCl}_{3}\right) \delta 202.46,155.00,136.43,133.28,130.22,121.67,113.17,44.67,36.96,34.98$, 34.24, 32.67, 28.74, 27.41, 26.74, 26.53, 26.39. HRMS (ESI) $\mathrm{m} / \mathrm{z}(\mathrm{M}+\mathrm{H})^{+}$calculated for $\mathrm{C}_{17} \mathrm{H}_{23} \mathrm{O}_{2}: 259.1698$, observed: 259.1697. The absolute configuration was assigned by X-ray analysis of its single crystal.

(R)-2-(cyclohexylmethyl)-7-fluoro-3,4-dihydronaphthalen-1(2H)-one $\quad$ (7). Colorless oil. Yield: $55 \%$. Enantiomeric excess: $88 \%$, determined by HPLC (CHIRALPAK IE, hexane/ isopropanol $=94 / 6$, flow rate 1.0 $\mathrm{mL} / \mathrm{min}, \mathrm{T}=30^{\circ} \mathrm{C}, 254 \mathrm{~nm}$ ): $\mathrm{t}_{\mathrm{R}}=6.176 \mathrm{~min}$ (minor), $\mathrm{t}_{\mathrm{R}}=6.465 \mathrm{~min}$ (major). $[\alpha]_{\mathrm{D}}{ }^{20}=5.0$ (c $\left.0.47, \mathrm{CHCl}_{3}\right) .{ }^{1} \mathbf{H}$ NMR $(400 \mathrm{MHz}$, Chloroform- $d$ ) $\delta 7.67(\mathrm{dd}, J=9.3,2.8 \mathrm{~Hz}, 1 \mathrm{H}), 7.21(\mathrm{dd}, J=8.5,5.2 \mathrm{~Hz}, 1 \mathrm{H}), 7.15(\mathrm{td}, J=8.3$, $2.8 \mathrm{~Hz}, 1 \mathrm{H}), 3.02-2.85(\mathrm{~m}, 2 \mathrm{H}), 2.56(\mathrm{ddt}, J=10.0,8.3,4.9 \mathrm{~Hz}, 1 \mathrm{H}), 2.22(\mathrm{dq}, J=14.3,4.8 \mathrm{~Hz}, 1 \mathrm{H}), 1.90-1.79$ (m, 2H), $1.76-1.62(\mathrm{~m}, 5 \mathrm{H}), 1.45-1.36(\mathrm{~m}, 1 \mathrm{H}), 1.31-1.15(\mathrm{~m}, 4 \mathrm{H}), 1.01-0.84(\mathrm{~m}, 2 \mathrm{H}) .{ }^{13} \mathrm{C} \mathrm{NMR}(101 \mathrm{MHz}$, $\left.\mathrm{CDCl}_{3}\right) \delta 200.08(\mathrm{~d}, J=1.8 \mathrm{~Hz}), 161.63(\mathrm{~d}, J=245.8 \mathrm{~Hz}), 139.65(\mathrm{~d}, J=3.1 \mathrm{~Hz}), 134.23(\mathrm{~d}, J=6.0 \mathrm{~Hz}), 130.54$ (d, $J=7.0 \mathrm{~Hz}), 120.49$ (d, $J=22.1 \mathrm{~Hz}), 113.41$ (d, $J=21.7 \mathrm{~Hz}), 44.42,36.89,34.93,34.22,32.69,28.50,27.60$, 26.72, 26.51, 26.37. HRMS (ESI) $\mathrm{m} / \mathrm{z}(\mathrm{M}+\mathrm{H})^{+}$calculated for $\mathrm{C}_{17} \mathrm{H}_{22} \mathrm{OF}: 261.1655$, observed: 261.1647. The absolute configuration was tentatively assigned by analogy.

(R)-7-chloro-2-(cyclohexylmethyl)-3,4-dihydronaphthalen-1(2H)-one (8). Colorless oil. Yield: 57\%. Enantiomeric excess: 85\%, determined by HPLC (CHIRALPAK IF, hexane/ isopropanol $=97 / 3$, flow rate 1.0 $\mathrm{mL} / \mathrm{min}, \mathrm{T}=30^{\circ} \mathrm{C}, 254 \mathrm{~nm}$ ): $\mathrm{t}_{\mathrm{R}}=6.309 \mathrm{~min}$ (minor), $\mathrm{t}_{\mathrm{R}}=7.025 \mathrm{~min}$ (major). $[\alpha]_{\mathrm{D}}{ }^{20}=-3.3$ (c $0.80, \mathrm{CHCl}_{3}$ ). ${ }^{1} \mathbf{H}$ NMR (400 MHz, Chloroform- $d$ ) $\delta 7.97(\mathrm{~d}, J=2.4 \mathrm{~Hz}, 1 \mathrm{H}), 7.40(\mathrm{dd}, J=8.2,2.4 \mathrm{~Hz}, 1 \mathrm{H}), 7.18(\mathrm{~d}, J=8.2 \mathrm{~Hz}$, $1 \mathrm{H}), 3.04-2.80(\mathrm{~m}, 2 \mathrm{H}), 2.65-2.46(\mathrm{~m}, 1 \mathrm{H}), 2.27-2.12(\mathrm{~m}, 1 \mathrm{H}), 1.90-1.76(\mathrm{~m}, 2 \mathrm{H}), 1.76-1.60(\mathrm{~m}, 5 \mathrm{H}), 1.45$ $-1.33(\mathrm{~m}, 1 \mathrm{H}), 1.29-1.12(\mathrm{~m}, 4 \mathrm{H}), 1.02-0.81(\mathrm{~m}, 2 \mathrm{H}) .{ }^{13} \mathbf{C} \mathbf{N M R}\left(101 \mathrm{MHz}, \mathrm{CDCl}_{3}\right) \delta 199.90,142.17,133.89$, 133.05, 132.83, 130.35, 127.34, 44.49, 36.86, 34.91, 34.21, 32.69, 28.26, 27.71, 26.72, 26.51, 26.37. HRMS (ESI) $\mathrm{m} / \mathrm{z}(\mathrm{M}+\mathrm{H})^{+}$calculated for $\mathrm{C}_{17} \mathrm{H}_{22} \mathrm{OCl}$ : 277.1359, observed: 277.1352. The absolute configuration was tentatively assigned by analogy.

(R)-7-bromo-2-(cyclohexylmethyl)-3,4-dihydronaphthalen-1(2H)-one $\quad(\mathbf{9}) . \quad$ Colorless $\quad$ oil. Yield: $70 \%$. Enantiomeric excess: 84\%, determined by HPLC (CHIRALPAK IC, hexane/ isopropanol $=97 / 3$, flow rate 1.0 $\mathrm{mL} / \mathrm{min}, \mathrm{T}=30^{\circ} \mathrm{C}, 254 \mathrm{~nm}$ ): $\mathrm{t}_{\mathrm{R}}=6.085 \mathrm{~min}$ (minor), $\mathrm{t}_{\mathrm{R}}=6.372 \mathrm{~min}$ (major). $[\alpha]_{\mathrm{D}}{ }^{20}=-7.1$ (c $\left.0.70, \mathrm{CHCl}_{3}\right) .{ }^{1} \mathbf{H}$ NMR (400 MHz, Chloroform- $d$ ) $\delta 8.12(\mathrm{~d}, J=2.2 \mathrm{~Hz}, 1 \mathrm{H}), 7.54(\mathrm{dd}, J=8.1,2.2 \mathrm{~Hz}, 1 \mathrm{H}), 7.11(\mathrm{~d}, J=8.2 \mathrm{~Hz}$, $1 \mathrm{H}), 3.00-2.79(\mathrm{~m}, 2 \mathrm{H}), 2.56(\mathrm{ddt}, J=10.1,8.2,4.9 \mathrm{~Hz}, 1 \mathrm{H}), 2.22(\mathrm{dq}, J=13.5,4.9 \mathrm{~Hz}, 1 \mathrm{H}), 1.83$ (ddt, $J=14.4$, $8.9,5.5 \mathrm{~Hz}, 2 \mathrm{H}), 1.76-1.60(\mathrm{~m}, 5 \mathrm{H}), 1.39$ (dddq, $J=11.2,8.2,5.4,2.9 \mathrm{~Hz}, 1 \mathrm{H}), 1.34-1.11(\mathrm{~m}, 4 \mathrm{H}), 1.05-0.83$ (m, 2H). ${ }^{13} \mathrm{C}$ NMR $\left(126 \mathrm{MHz}, \mathrm{CDCl}_{3}\right) \delta 199.74,142.63,135.89,134.16,130.62,130.39,120.67,44.48,36.85$, 34.91, 34.20, 32.69, 28.19, 27.76, 26.71, 26.51, 26.37. HRMS (ESI) $\mathrm{m} / \mathrm{z}(\mathrm{M}+\mathrm{H})^{+}$calculated for $\mathrm{C}_{17} \mathrm{H}_{22} \mathrm{OBr}$ : 321.0854, observed: 321.0847 . The absolute configuration was tentatively assigned by analogy.

(R)-2-(cyclohexylmethyl)-6-fluoro-3,4-dihydronaphthalen-1(2H)-one (10) .Colorless oil. Yield: 55\%. Enantiomeric excess: 90\%, determined by HPLC (CHIRALPAK IF, hexane/ isopropanol $=97 / 3$, flow rate 1.0 
$\mathrm{mL} / \mathrm{min}, \mathrm{T}=30^{\circ} \mathrm{C}, 254 \mathrm{~nm}$ ): $\mathrm{t}_{\mathrm{R}}=8.100 \mathrm{~min}$ (minor), $\mathrm{t}_{\mathrm{R}}=9.333 \mathrm{~min}$ (major). $[\alpha]_{\mathrm{D}}{ }^{20}=0.66$ (c $\left.0.77, \mathrm{CHCl}_{3}\right) .{ }^{1} \mathbf{H}$ NMR (400 MHz, Chloroform- $d$ ) $\delta 8.04(\mathrm{dd}, J=8.7,6.1 \mathrm{~Hz}, 1 \mathrm{H}), 6.97(\mathrm{td}, J=8.5,2.6 \mathrm{~Hz}, 1 \mathrm{H}), 6.90(\mathrm{dd}, J=9.2$, $2.6 \mathrm{~Hz}, 1 \mathrm{H}), 3.04-2.87(\mathrm{~m}, 2 \mathrm{H}), 2.63-2.50(\mathrm{~m}, 1 \mathrm{H}), 2.28-2.14(\mathrm{~m}, 1 \mathrm{H}), 1.91-1.78(\mathrm{~m}, 2 \mathrm{H}), 1.79-1.61(\mathrm{~m}$, 5H), $1.47-1.36(\mathrm{~m}, 1 \mathrm{H}), 1.34-1.12(\mathrm{~m}, 4 \mathrm{H}), 1.06-0.80(\mathrm{~m}, 2 \mathrm{H}){ }^{13} \mathbf{C} \mathbf{~ N M R}\left(126 \mathrm{MHz}, \mathrm{CDCl}_{3}\right) \delta 199.64$, $165.64(\mathrm{~d}, J=254.8 \mathrm{~Hz}), 146.97(\mathrm{~d}, J=9.1 \mathrm{~Hz}), 130.66(\mathrm{~d}, J=9.8 \mathrm{~Hz}), 129.31(\mathrm{~d}, J=2.7 \mathrm{~Hz}), 114.96(\mathrm{~d}, J=$ $21.2 \mathrm{~Hz}), 114.32(\mathrm{~d}, J=22.0 \mathrm{~Hz}), 44.47,36.93,34.97,34.24,32.70,28.45$ (d, $J=1.6 \mathrm{~Hz}) 28.37,26.73,26.52$, 26.38. HRMS (ESI) $\mathrm{m} / \mathrm{z}(\mathrm{M}+\mathrm{H})^{+}$calculated for $\mathrm{C}_{17} \mathrm{H}_{22} \mathrm{OF}$ : 261.1655, observed: 261.1652. The absolute configuration was tentatively assigned by analogy.

((R)-6-chloro-2-(cyclohexylmethyl)-3,4-dihydronaphthalen-1(2H)-one (11). Colorless oil. Yield: 63\%. Enantiomeric excess: 84\%, determined by HPLC (CHIRALPAK IE, hexane/ isopropanol $=99 / 1$, flow rate 1.0 $\mathrm{mL} / \mathrm{min}, \mathrm{T}=30^{\circ} \mathrm{C}, 254 \mathrm{~nm}$ ): $\mathrm{t}_{\mathrm{R}}=9.093 \mathrm{~min}$ (minor), $\mathrm{t}_{\mathrm{R}}=9.597 \mathrm{~min}$ (major). $[\alpha]_{\mathrm{D}}{ }^{20}=5.3$ (c $0.57, \mathrm{CHCl}_{3}$ ). ${ }^{1} \mathbf{H}$ NMR (400 MHz, Chloroform- $d$ ) $\delta 7.95(\mathrm{~d}, J=8.4 \mathrm{~Hz}, 1 \mathrm{H}), 7.25$ (dd, $J=7.8,2.4 \mathrm{~Hz}, 1 \mathrm{H}), 7.23(\mathrm{~s}, 1 \mathrm{H}), 3.01-$ $2.87(\mathrm{~m}, 2 \mathrm{H}), 2.56(\mathrm{ddt}, J=10.1,8.3,4.9 \mathrm{~Hz}, 1 \mathrm{H}), 2.28-2.18(\mathrm{~m}, 1 \mathrm{H}), 1.90-1.79(\mathrm{~m}, 2 \mathrm{H}), 1.77-1.61(\mathrm{~m}, 5 \mathrm{H})$, $1.45-1.35(\mathrm{~m}, 1 \mathrm{H}), 1.32-1.10(\mathrm{~m}, 4 \mathrm{H}), 1.04-0.82(\mathrm{~m}, 2 \mathrm{H}) .{ }^{13} \mathbf{C} \mathbf{N M R}\left(101 \mathrm{MHz}, \mathrm{CDCl}_{3}\right) \delta$ 200.03, 145.55, 139.39, 131.09, 129.30, 128.59, 127.18, 44.57, 36.91, 34.94, 34.22, 32.69, 28.28, 28.14, 26.72, 26.51, 26.37. HRMS (ESI) $\mathrm{m} / \mathrm{z}(\mathrm{M}+\mathrm{H})^{+}$calculated for $\mathrm{C}_{17} \mathrm{H}_{22} \mathrm{OCl}$ : 277.1359, observed: 277.1358. The absolute configuration was tentatively assigned by analogy.

(R)-2-(cyclohexylmethyl)-5-hydroxy-3,4-dihydronaphthalen-1(2H)-one (12). Colorless oil. Yield: 58\%. Enantiomeric excess: 85\%, determined by HPLC (CHIRALPAK AD, hexane/ isopropanol $=97 / 3$, flow rate 1.0 $\mathrm{mL} / \mathrm{min}, \mathrm{T}=30^{\circ} \mathrm{C}, 254 \mathrm{~nm}$ ): $\mathrm{t}_{\mathrm{R}}=20.183 \min$ (minor), $\mathrm{t}_{\mathrm{R}}=27.664 \mathrm{~min}$ (major). $[\alpha]_{\mathrm{D}}{ }^{20}=5.0$ (c 1.3, $\mathrm{CHCl}_{3}$ ). ${ }^{1} \mathbf{H}$ NMR (500 MHz, Chloroform- $d$ ) $\delta 7.62(\mathrm{dd}, J=7.8,1.2 \mathrm{~Hz}, 1 \mathrm{H}), 7.16(\mathrm{t}, J=7.9 \mathrm{~Hz}, 1 \mathrm{H}), 7.03(\mathrm{dd}, J=7.8,1.2$ $\mathrm{Hz}, 1 \mathrm{H}), 6.27$ (s, 1H), 3.03 (ddd, $J=17.4,6.1,4.8 \mathrm{~Hz}, 1 \mathrm{H}), 2.82$ (ddd, $J=17.4,9.0,4.9 \mathrm{~Hz}, 1 \mathrm{H}), 2.62$ (dddd, $J=$ 9.9, 8.2, 5.6, $4.3 \mathrm{~Hz}, 1 \mathrm{H}), 2.24(\mathrm{ddt}, J=13.6,6.2,4.7 \mathrm{~Hz}, 1 \mathrm{H}), 1.91-1.78(\mathrm{~m}, 2 \mathrm{H}), 1.75-1.61(\mathrm{~m}, 5 \mathrm{H}), 1.45-$ $1.36(\mathrm{~m}, 1 \mathrm{H}), 1.33-1.28(\mathrm{~m}, 1 \mathrm{H}), 1.26-1.11(\mathrm{~m}, 3 \mathrm{H}), 1.01-0.82(\mathrm{~m}, 2 \mathrm{H}) .{ }^{13} \mathbf{C} \mathbf{N M R}\left(126 \mathrm{MHz}, \mathrm{CDCl}_{3}\right) \delta$ 202.44, 153.44, 133.78, 130.81, 126.98, 119.66, 119.61, 44.18, 36.84, 34.96, 34.17, 32.75, 27.55, 26.72, 26.48, 26.35, 21.41. HRMS (ESI) $\mathrm{m} / \mathrm{z}(\mathrm{M}+\mathrm{H})^{+}$calculated for $\mathrm{C}_{17} \mathrm{H}_{22} \mathrm{O}_{2}: 259.1698$, observed: 259.1698 . The absolute configuration was tentatively assigned by analogy.

(R)-5-chloro-2-(cyclohexylmethyl)-3,4-dihydronaphthalen-1(2H)-one (13). Colorless oil. Yield: $47 \%$. Enantiomeric excess: $81 \%$, determined by HPLC (CHIRALPAK ID, hexane/ isopropanol $=99 / 1$, flow rate 1.0 $\mathrm{mL} / \mathrm{min}, \mathrm{T}=30^{\circ} \mathrm{C}, 254 \mathrm{~nm}$ ): $\mathrm{t}_{\mathrm{R}}=6.640 \mathrm{~min}$ (minor), $\mathrm{t}_{\mathrm{R}}=6.886 \mathrm{~min}$ (major). $[\alpha]_{\mathrm{D}}{ }^{20}=3.2$ (c $0.80, \mathrm{CHCl}_{3}$ ). ${ }^{1} \mathbf{H}$ NMR (400 MHz, Chloroform- $d$ ) $\delta 7.94(\mathrm{dd}, J=7.7,1.3 \mathrm{~Hz}, 1 \mathrm{H}), 7.53(\mathrm{dd}, J=7.9,1.4 \mathrm{~Hz}, 1 \mathrm{H}), 7.25(\mathrm{t}, J=7.9$ $\mathrm{Hz}, 1 \mathrm{H}), 3.12(\mathrm{dt}, J=17.9,5.2 \mathrm{~Hz}, 1 \mathrm{H}), 2.91$ (ddd, $J=17.8,9.4,5.0 \mathrm{~Hz}, 1 \mathrm{H}), 2.59$ (dddd, $J=10.1,8.2,5.6,4.3$ $\mathrm{Hz}, 1 \mathrm{H}), 2.25(\mathrm{dq}, J=13.7,5.0 \mathrm{~Hz}, 1 \mathrm{H}), 1.84$ (dddd, $J=22.5,13.8,9.2,5.2 \mathrm{~Hz}, 2 \mathrm{H}), 1.74-1.60(\mathrm{~m}, 5 \mathrm{H}), 1.46-$ $1.36(\mathrm{~m}, 1 \mathrm{H}), 1.28-1.11(\mathrm{~m}, 4 \mathrm{H}), 0.98-0.81(\mathrm{~m}, 2 \mathrm{H}) .{ }^{13} \mathbf{C} \mathbf{~ N M R}\left(101 \mathrm{MHz}, \mathrm{CDCl}_{3}\right) \delta 200.24,141.28,134.48$, 134.06, 133.68, 127.34, 126.17, 43.78, 36.72, 34.93, 34.16, 32.78, 27.40, 26.71, 26.49, 26.36, 25.76.HRMS (ESI) $\mathrm{m} / \mathrm{z}(\mathrm{M}+\mathrm{H})^{+}$calculated for $\mathrm{C}_{17} \mathrm{H}_{22} \mathrm{OCl}: 277.1359$, observed: 277.1361 . The absolute configuration was tentatively assigned by analogy.

(R)-3-(cyclohexylmethyl)chroman-4-one (14). Colorless oil. Yield: 67\%. Enantiomeric excess: 83\%, determined by HPLC (CHIRALPAK IC, hexane/ isopropanol $=99 / 1$, flow rate $1.0 \mathrm{~mL} / \mathrm{min}, \mathrm{T}=30^{\circ} \mathrm{C}, 254 \mathrm{~nm}$ ): $\mathrm{t}_{\mathrm{R}}=7.990$ 
$\min$ (minor), $\mathrm{t}_{\mathrm{R}}=7.502 \mathrm{~min}$ (major). $[\alpha]_{\mathrm{D}}{ }^{20}=-11\left(\mathrm{c} 0.57, \mathrm{CHCl}_{3}\right) .{ }^{1} \mathbf{H} \mathbf{N M R}(400 \mathrm{MHz}$, Chloroform- $d$ ) $\delta 7.88$ (dd, $J=7.8,1.7 \mathrm{~Hz}, 1 \mathrm{H}), 7.45(\mathrm{td}, J=8.6,1.8 \mathrm{~Hz}, 1 \mathrm{H}), 7.00(\mathrm{t}, J=7.5 \mathrm{~Hz}, 1 \mathrm{H}), 6.94(\mathrm{~d}, J=8.3 \mathrm{~Hz}, 1 \mathrm{H}), 4.49(\mathrm{dd}, J=$ 11.4, $4.3 \mathrm{~Hz}, 1 \mathrm{H}), 4.23(\mathrm{dd}, J=11.4,8.4 \mathrm{~Hz}, 1 \mathrm{H}), 2.76(\mathrm{tdd}, J=8.2,5.7,4.3 \mathrm{~Hz}, 1 \mathrm{H}), 1.82-1.59(\mathrm{~m}, 7 \mathrm{H}), 1.46-$ $1.37(\mathrm{~m}, 1 \mathrm{H}), 1.35-1.11(\mathrm{~m}, 4 \mathrm{H}), 1.02-0.85(\mathrm{~m}, 2 \mathrm{H}) .{ }^{13} \mathbf{C} \mathbf{N M R}\left(101 \mathrm{MHz}, \mathrm{CDCl}_{3}\right) \delta 195.15,161.50,135.76$, 127.54, 121.41, 120.73, 117.74, 77.48, 76.84, 70.77, 43.32, 35.03, 33.87, 33.74, 32.86, 26.60, 26.35, 26.25. HRMS (ESI) $\mathrm{m} / \mathrm{z}(\mathrm{M}+\mathrm{H})^{+}$calculated for $\mathrm{C}_{16} \mathrm{H}_{21} \mathrm{O}_{2}$ : 245.1542, observed: 245.1537. The absolute configuration was tentatively assigned by analogy.

(R)-5-(cyclohexylmethyl)-6,7-dihydrobenzo[b]thiophen-4(5H)-one $\quad$ (15). Colorless $\quad$ oil. $\quad$ Yield: $84 \%$. Enantiomeric excess: 85\%, determined by HPLC (CHIRALPAK IE, hexane/ isopropanol $=97 / 3$, flow rate 1.0 $\mathrm{mL} / \mathrm{min}, \mathrm{T}=30^{\circ} \mathrm{C}, 254 \mathrm{~nm}$ ): $\mathrm{t}_{\mathrm{R}}=10.825 \min \left(\right.$ minor), $\mathrm{t}_{\mathrm{R}}=11.391 \mathrm{~min}$ (major). $[\alpha]_{\mathrm{D}}{ }^{20}=5.0\left(\mathrm{c} 0.87, \mathrm{CHCl}_{3}\right) .{ }^{1} \mathbf{H}$ NMR (400 MHz, Chloroform- $d$ ) $\delta 7.37$ (d, $J=5.3 \mathrm{~Hz}, 1 \mathrm{H}), 7.04$ (d, $J=5.3 \mathrm{~Hz}, 1 \mathrm{H}), 3.09$ (ddd, $J=17.1,6.1,4.9$ Hz, 1H), 2.99 (ddd, $J=17.1,8.6,4.8 \mathrm{~Hz}, 1 \mathrm{H}$ ), 2.54 (tt, $J=9.3,4.9 \mathrm{~Hz}, 1 \mathrm{H}$ ), 2.29 (ddt, $J=13.4,6.1,4.6 \mathrm{~Hz}, 1 \mathrm{H}$ ), $1.95(\mathrm{dtd}, J=13.7,9.0,4.9 \mathrm{~Hz}, 1 \mathrm{H}), 1.82(\mathrm{ddd}, J=13.9,8.5,5.4 \mathrm{~Hz}, 1 \mathrm{H}), 1.76-1.61(\mathrm{~m}, 5 \mathrm{H}), 1.45-1.33(\mathrm{~m}$, $1 \mathrm{H}), 1.30-1.11(\mathrm{~m}, 4 \mathrm{H}), 1.02-0.82(\mathrm{~m}, 2 \mathrm{H}) .{ }^{13} \mathbf{C} \mathbf{~ N M R}\left(126 \mathrm{MHz}, \mathrm{CDCl}_{3}\right) \delta 196.14,154.75,137.05,125.32$, 123.21, 43.60, 36.58, 35.12, 34.21, 32.66, 29.77, 26.73, 26.52, 26.38, 24.18. HRMS (ESI) m/z (M+H) ${ }^{+}$calculated for $\mathrm{C}_{15} \mathrm{H}_{21} \mathrm{OS}$ : 249.1313, observed: 249.1305 . The absolute configuration was tentatively assigned by analogy.

((R)-6-(cyclohexylmethyl)-6,7,8,9-tetrahydro-5H-benzo[7]annulen-5-one (16). Colorless oil. Yield: 44\%. Enantiomeric excess: 91\%, determined by HPLC (CHIRALPAK IE, hexane/ isopropanol $=99 / 1$, flow rate 1.0 $\mathrm{mL} / \mathrm{min}, \mathrm{T}=30^{\circ} \mathrm{C}, 240 \mathrm{~nm}$ ): $\mathrm{t}_{\mathrm{R}}=10.335 \mathrm{~min}$ (minor), $\mathrm{t}_{\mathrm{R}}=9.549 \min$ (major). $[\alpha]_{\mathrm{D}}{ }^{20}=-92.0$ (c $\left.0.73, \mathrm{CHCl}_{3}\right) .{ }^{1} \mathbf{H}$ NMR $(400 \mathrm{MHz}$, Chloroform- $d$ ) $\delta 7.61(\mathrm{dd}, J=7.7,1.5 \mathrm{~Hz}, 1 \mathrm{H}), 7.36(\mathrm{td}, J=7.5,1.6 \mathrm{~Hz}, 1 \mathrm{H}), 7.27(\mathrm{td}, J=7.4$, $1.1 \mathrm{~Hz}, 1 \mathrm{H}), 7.22(\mathrm{~d}, J=7.5 \mathrm{~Hz}, 1 \mathrm{H}), 3.07-2.90(\mathrm{~m}, 3 \mathrm{H}), 2.07$ (ddq, $J=12.8,6.2,3.0 \mathrm{~Hz}, 1 \mathrm{H}), 1.97-1.83(\mathrm{~m}$, 2H), $1.70-1.50(\mathrm{~m}, 7 \mathrm{H}), 1.34-1.05(\mathrm{~m}, 5 \mathrm{H}), 0.94-0.79(\mathrm{~m}, 2 \mathrm{H}) .{ }^{13} \mathbf{C} \mathbf{N M R}\left(101 \mathrm{MHz}, \mathrm{CDCl}_{3}\right) \delta 208.07$, 142.12, 140.68, 131.03, 129.95, 128.25, 126.43, 47.20, 38.54, 35.71, 33.99, 33.79, 33.46, 31.26, 26.68, 26.46, 26.43, 25.64. HRMS (ESI) $\mathrm{m} / \mathrm{z}(\mathrm{M}+\mathrm{H})^{+}$calculated for $\mathrm{C}_{18} \mathrm{H}_{25} \mathrm{O}: 257.1905$, observed: 257.1904. The absolute configuration was tentatively assigned by analogy.

(S)-5-(cyclohexylmethyl)-2-phenylcyclopent-2-en-1-one (17). White solid. Yield: 61\%. Enantiomeric excess: $84 \%$, determined by HPLC (CHIRALPAK IE, hexane/ isopropanol $=90 / 10$, flow rate $1.0 \mathrm{~mL} / \mathrm{min}, \mathrm{T}=30^{\circ} \mathrm{C}, 254$ $\mathrm{nm}): \mathrm{t}_{\mathrm{R}}=7.999 \min$ (minor), $\mathrm{t}_{\mathrm{R}}=8.438 \mathrm{~min}$ (major). $[\alpha]_{\mathrm{D}^{20}}=-83.5$ (c $\left.0.63, \mathrm{CHCl}_{3}\right) .{ }^{1} \mathbf{H} \mathbf{~ N M R}(400 \mathrm{MHz}$, Chloroform- $d$ ) $\delta 7.77$ (t, $J=3.0 \mathrm{~Hz}, 1 \mathrm{H}$ ), $7.74-7.66$ (m, 2H), $7.42-7.28$ (m, 3H), 2.89 (ddd, $J=19.5,6.6,3.1$ Hz, 1H), 2.60 (dddd, $J=10.8,6.6,4.2,2.4 \mathrm{~Hz}, 1 \mathrm{H}), 2.37$ (dt, $J=19.4,2.7 \mathrm{~Hz}, 1 \mathrm{H}), 1.86-1.77$ (m, $2 \mathrm{H}), 1.77-$ $1.62(\mathrm{~m}, 4 \mathrm{H}), 1.48-1.37(\mathrm{~m}, 1 \mathrm{H}), 1.31-1.15(\mathrm{~m}, 4 \mathrm{H}), 1.06-0.89(\mathrm{~m}, 2 \mathrm{H}) .{ }^{13} \mathbf{C} \mathbf{~ N M R}\left(101 \mathrm{MHz}, \mathrm{CDCl}_{3}\right) \delta$ 210.19, 157.46, 142.62, 131.97, 128.50, 128.38, 127.14, , 44.61, 39.62, 36.17, 34.29, 34.02, 32.61, 26.65, 26.50, 26.37. HRMS (ESI) $\mathrm{m} / \mathrm{z}(\mathrm{M}+\mathrm{H})^{+}$calculated for $\mathrm{C}_{18} \mathrm{H}_{23} \mathrm{O}: 255.1749$, observed: 255.1743 . The absolute configuration was tentatively assigned by analogy.

(R)-3-(cyclohexylmethyl)-4,5-dihydro-[1,1'-biphenyl]-2(3H)-one (18). Colorless oil. Yield: 63\%. Enantiomeric excess: 73\%, determined by HPLC (CHIRALPAK IE, hexane/ isopropanol $=90 / 10$, flow rate $1.0 \mathrm{~mL} / \mathrm{min}, \mathrm{T}=$ $30^{\circ} \mathrm{C}, 254 \mathrm{~nm}$ ): $\mathrm{t}_{\mathrm{R}}=6.636 \mathrm{~min}$ (minor), $\mathrm{t}_{\mathrm{R}}=6.950 \mathrm{~min}$ (major). $[\alpha]_{\mathrm{D}}{ }^{20}=-3.9\left(\mathrm{c} 0.6, \mathrm{CHCl}_{3}\right) .{ }^{1} \mathbf{H} \mathbf{~ N M R}(500 \mathrm{MHz}$, Chloroform- $d$ ) $\delta 7.35-7.28(\mathrm{~m}, 5 \mathrm{H}), 6.94(\mathrm{t}, J=4.2 \mathrm{~Hz}, 1 \mathrm{H}), 2.62-2.48(\mathrm{~m}, 3 \mathrm{H}), 2.18(\mathrm{td}, J=10.1,5.1 \mathrm{~Hz}, 1 \mathrm{H}), 1.87$ - $1.80(\mathrm{~m}, 2 \mathrm{H}), 1.75-1.62(\mathrm{~m}, 5 \mathrm{H}), 1.41-1.33(\mathrm{~m}, 1 \mathrm{H}), 1.30-1.14(\mathrm{~m}, 4 \mathrm{H}), 1.01-0.83(\mathrm{~m}, 2 \mathrm{H}) .{ }^{13} \mathbf{C}$ NMR $(126$ 
$\left.\mathrm{MHz}, \mathrm{CDCl}_{3}\right) \delta 201.09,146.56,139.92,137.03,128.77,128.06,127.56, \quad 44.37,36.96,35.06,34.14,32.86,28.10$, 26.73, 26.51, 26.39, 25.41. HRMS (ESI) m/z (M+H) ${ }^{+}$calculated for $\mathrm{C}_{19} \mathrm{H}_{25} \mathrm{O}: 269.1905$, observed: 269.1898. The absolute configuration was tentatively assigned by analogy.

(S)-6-(cyclohexylmethyl)-4,4-dimethylcyclohex-2-en-1-one (19). Colorless oil. Yield: 81\%. Enantiomeric excess: 93\%, determined by HPLC (CHIRALPAK IE, hexane/ isopropanol = 99.5/0.5, flow rate $0.5 \mathrm{~mL} / \mathrm{min}, \mathrm{T}=$ $\left.30^{\circ} \mathrm{C}, 220 \mathrm{~nm}\right): \mathrm{t}_{\mathrm{R}}=15.091 \mathrm{~min}$ (minor), $\mathrm{t}_{\mathrm{R}}=15.995 \mathrm{~min}$ (major). $[\alpha]_{\mathrm{D}}{ }^{20}=-3.5$ (c $\left.0.87, \mathrm{CHCl}_{3}\right) .{ }^{1} \mathbf{H} \mathbf{~ N M R}(400$ MHz, Chloroform- $d$ ) $\delta 6.57(\mathrm{dd}, J=10.0,2.2 \mathrm{~Hz}, 1 \mathrm{H}), 5.81(\mathrm{~d}, J=10.0 \mathrm{~Hz}, 1 \mathrm{H}), 2.48(\mathrm{ddt}, J=13.5,9.0,4.6 \mathrm{~Hz}$, 1H), $1.95-1.79(\mathrm{~m}, 2 \mathrm{H}), 1.75-1.60(\mathrm{~m}, 5 \mathrm{H}), 1.54(\mathrm{t}, J=13.5 \mathrm{~Hz}, 1 \mathrm{H}), 1.39-1.16(\mathrm{~m}, 7 \mathrm{H}), 1.18(\mathrm{~s}, 3 \mathrm{H}), 1.14(\mathrm{~s}$, $3 \mathrm{H}), 1.11-0.92(\mathrm{~m}, 2 \mathrm{H}), 0.89-0.79(\mathrm{~m}, 1 \mathrm{H}) .{ }^{13} \mathbf{C} \mathbf{N M R}\left(126 \mathrm{MHz}, \mathrm{CDCl}_{3}\right) \delta 202.30,158.35,126.93,42.49$, $39.89,36.58,34.63,34.47,33.69,32.32,30.73,26.77,26.56,26.41,25.57$. HRMS (ESI) m/z (M+H) ${ }^{+}$calculated for $\mathrm{C}_{15} \mathrm{H}_{25} \mathrm{O}: 221.1905$, observed: 221.1893. The absolute configuration was tentatively assigned by analogy.

(R)-7-(cyclohexylmethyl)cyclohept-2-en-1-one (20). Colorless oil. Yield: 59\%. Enantiomeric excess: 84\%, determined by HPLC (CHIRALPAK ID, hexane/ isopropanol =97/3, flow rate $1.0 \mathrm{~mL} / \mathrm{min}, \mathrm{T}=30^{\circ} \mathrm{C}, 237 \mathrm{~nm}$ ): $\mathrm{t}_{\mathrm{R}}=6.934 \min$ (minor), $\mathrm{t}_{\mathrm{R}}=5.705$ min (major). $[\alpha]_{\mathrm{D}}{ }^{20}=-22.4$ (c 0.41, $\left.\mathrm{CHCl}_{3}\right) .{ }^{1} \mathbf{H} \mathbf{N M R}(500 \mathrm{MHz}, \mathrm{Chloroform}-d)$ $\delta 6.59(\mathrm{ddd}, J=11.3,6.8,3.8 \mathrm{~Hz}, 1 \mathrm{H}), 6.02(\mathrm{dd}, J=12.0,2.4 \mathrm{~Hz}, 1 \mathrm{H}), 2.76(\mathrm{dq}, J=12.4,6.6 \mathrm{~Hz}, 1 \mathrm{H}), 2.50-$ $2.33(\mathrm{~m}, 2 \mathrm{H}), 1.87(\mathrm{dp}, J=12.1,5.8 \mathrm{~Hz}, 2 \mathrm{H}), 1.75-1.58(\mathrm{~m}, 7 \mathrm{H}), 1.43(\mathrm{ddd}, J=14.8,10.3,4.3 \mathrm{~Hz}, 1 \mathrm{H}), 1.32-$ $1.06(\mathrm{~m}, 5 \mathrm{H}), 0.94-0.74(\mathrm{~m}, 2 \mathrm{H}) .{ }^{13} \mathbf{C} \mathbf{N M R}^{13}\left(126 \mathrm{MHz}, \mathrm{CDCl}_{3}\right) \delta 206.74,145.86,133.02,48.93,39.08,35.17$, 33.80, 33.29, 30.10, 29.95, 26.72, 26.46, 26.41, 25.50. HRMS (EI) m/z (M-e) ${ }^{+}$calculated for $\mathrm{C}_{14} \mathrm{H}_{22} \mathrm{O}: 206.1665$, observed: 206.1673. The absolute configuration was tentatively assigned by analogy.

(R)-3-cyclohexyl-2-methyl-1-phenylpropan-1-one (21). Colorless oil. Yield: 35\%. Enantiomeric excess: 39\%, determined by HPLC (CHIRALPAK ID, hexane/ isopropanol =97/3, flow rate $\left.1 \mathrm{~mL} / \mathrm{min}, \mathrm{T}=30^{\circ} \mathrm{C}, 240 \mathrm{~nm}\right): \mathrm{t}_{\mathrm{R}}=$ $4.841 \min$ (minor), $\mathrm{t}_{\mathrm{R}}=4.478 \mathrm{~min}$ (major). $[\alpha]_{\mathrm{D}}{ }^{20}=-1.2\left(\mathrm{c} 0.11, \mathrm{CHCl}_{3}\right)$. ${ }^{1} \mathbf{H} \mathbf{N M R}{ }^{1} \mathrm{H} \mathrm{NMR}(500 \mathrm{MHz}$, Chloroform- $d$ ) $\delta 7.96(\mathrm{~d}, J=7.6 \mathrm{~Hz}, 2 \mathrm{H}), 7.56(\mathrm{t}, J=7.5 \mathrm{~Hz}, 1 \mathrm{H}), 7.47(\mathrm{t}, J=7.6 \mathrm{~Hz}, 2 \mathrm{H}), 3.59(\mathrm{~h}, J=6.9 \mathrm{~Hz}, 1 \mathrm{H})$, $1.84-1.58(\mathrm{~m}, 6 \mathrm{H}), 1.36-1.23(\mathrm{~m}, 2 \mathrm{H}), 1.23-1.08(\mathrm{~m}, 6 \mathrm{H}), 0.97-0.80(\mathrm{~m}, 2 \mathrm{H}) .{ }^{\mathbf{1 3}} \mathbf{C ~ N M R}\left(126 \mathrm{MHz} \mathrm{CDCl}_{3}\right) \delta$ 204.79, 136.75, 132.93, 128.76, 128.39, 41.42, 37.81, 35.52, 34.01, 33.22, 26.67, 26.41, 26.35, 17.68. HRMS (EI) m/z $(\mathrm{M}-\mathrm{e})^{+}$calculated for $\mathrm{C}_{16} \mathrm{H}_{22} \mathrm{O}: 230.1665$, observed: 230.1663 . The absolute configuration was tentatively assigned by analogy.

(R)-2-(cyclopentylmethyl)-3,4-dihydronaphthalen-1(2H)-one (22). Colorless oil. Yield: 58\%. Enantiomeric excess: $88 \%$, determined by HPLC (CHIRALPAK IF, hexane/ isopropanol = 97/3, flow rate $1.0 \mathrm{~mL} / \mathrm{min}$, T = $30^{\circ} \mathrm{C}, 254 \mathrm{~nm}$ ): $\mathrm{t}_{\mathrm{R}}=6.703 \mathrm{~min}$ (minor), $\mathrm{t}_{\mathrm{R}}=7.226 \mathrm{~min}$ (major). $[\alpha]_{\mathrm{D}}{ }^{20}=5.2$ (c $\left.0.90, \mathrm{CHCl}_{3}\right) .{ }^{1} \mathbf{H} \mathbf{N M R}(400 \mathrm{MHz}$, Chloroform- $d$ ) $\delta 8.02(\mathrm{dd}, J=7.8,1.4 \mathrm{~Hz}, 1 \mathrm{H}), 7.45(\mathrm{td}, J=7.5,1.5 \mathrm{~Hz}, 1 \mathrm{H}), 7.29(\mathrm{td}, J=7.5,1.1 \mathrm{~Hz}, 1 \mathrm{H}), 7.22$ $(\mathrm{d}, J=7.3 \mathrm{~Hz}, 1 \mathrm{H}), 3.10-2.89(\mathrm{~m}, 2 \mathrm{H}), 2.59-2.44(\mathrm{~m}, 1 \mathrm{H}), 2.34-2.19(\mathrm{~m}, 1 \mathrm{H}), 2.03-1.93(\mathrm{~m}, 2 \mathrm{H}), 1.92-$ $1.72(\mathrm{~m}, 3 \mathrm{H}), 1.68-1.60(\mathrm{~m}, 2 \mathrm{H}), 1.61-1.41(\mathrm{~m}, 3 \mathrm{H}), 1.24-1.02(\mathrm{~m}, 2 \mathrm{H}) .{ }^{13} \mathbf{C ~ N M R}\left(126 \mathrm{MHz} \mathrm{CDCl}_{3}\right) \delta$ 200.90, 143.98, 133.16, 132.63, 128.75, 127.58, 126.64, 46.78, 37.64, 35.66, 33.44, 32.31, 28.46, 28.25, 25.27, 25.23. HRMS (ESI) $\mathrm{m} / \mathrm{z}(\mathrm{M}+\mathrm{H})^{+}$calculated for $\mathrm{C}_{16} \mathrm{H}_{21} \mathrm{O}$ : 229.1592, observed: 229.1589. The absolute configuration was tentatively assigned by analogy.

(R)-2-(cycloheptylmethyl)-3,4-dihydronaphthalen-1(2H)-one (23). Colorless oil. Yield: 61\%. Enantiomeric excess: 88\%, determined by HPLC (CHIRALPAK IE, hexane/ isopropanol = 97/3, flow rate $1.0 \mathrm{~mL} / \mathrm{min}$, $\mathrm{T}=$ $\left.30^{\circ} \mathrm{C}, 254 \mathrm{~nm}\right): \mathrm{t}_{\mathrm{R}}=8.307 \min ($ minor $), \mathrm{t}_{\mathrm{R}}=8.797 \mathrm{~min}$ (major). $[\alpha]_{\mathrm{D}}{ }^{20}=-3.4\left(\mathrm{c} 0.53, \mathrm{CHCl}_{3}\right) .{ }^{1} \mathbf{H ~ N M R}(400 \mathrm{MHz}$, 
Chloroform- $d$ ) $\delta 8.02(\mathrm{dd}, J=7.9,1.4 \mathrm{~Hz}, 1 \mathrm{H}), 7.45(\mathrm{td}, J=7.4,1.5 \mathrm{~Hz}, 1 \mathrm{H}), 7.29(\mathrm{td}, J=7.6,1.2 \mathrm{~Hz}, 1 \mathrm{H}), 7.22$ $(\mathrm{dd}, J=7.7,1.2 \mathrm{~Hz}, 1 \mathrm{H}), 3.06-2.86(\mathrm{~m}, 2 \mathrm{H}), 2.62-2.45(\mathrm{~m}, 1 \mathrm{H}), 2.30-2.14(\mathrm{~m}, 1 \mathrm{H}), 1.99-1.79(\mathrm{~m}, 2 \mathrm{H}), 1.74$ $-1.55(\mathrm{~m}, 7 \mathrm{H}), 1.54-1.41(\mathrm{~m}, 4 \mathrm{H}), 1.39-1.20(\mathrm{~m}, 3 \mathrm{H}) .{ }^{13} \mathbf{C} \mathbf{N M R}\left(126 \mathrm{MHz}, \mathrm{CDCl}_{3}\right) \delta 201.08,143.96,133.14$, 132.67, 128.74, 127.58, 126.63, 45.27, 37.47, 34.44, 33.30, 31.18, 28.50, 28.27, 27.53, 27.44, 26.41, 25.55, 25.48. HRMS (ESI) $\mathrm{m} / \mathrm{z}(\mathrm{M}+\mathrm{H})^{+}$calculated for $\mathrm{C}_{18} \mathrm{H}_{25} \mathrm{O}$ : 257.1905, observed: 257.1898. The absolute configuration was tentatively assigned by analogy.

(R)-2-(cyclooctylmethyl)-3,4-dihydronaphthalen-1(2H)-one (24). Colorless oil. Yield: $73 \%$. Enantiomeric excess: $92 \%$, determined by HPLC (CHIRALPAK IF, hexane/ isopropanol $=97 / 3$, flow rate $1.0 \mathrm{~mL} / \mathrm{min}, \mathrm{T}=$ $30^{\circ} \mathrm{C}, 254 \mathrm{~nm}$ ): $\mathrm{t}_{\mathrm{R}}=9.926 \mathrm{~min}\left(\right.$ minor), $\mathrm{t}_{\mathrm{R}}=7.440 \mathrm{~min}$ (major). $[\alpha]_{\mathrm{D}}{ }^{20}=-1.0\left(\mathrm{c} 1.3, \mathrm{CHCl}_{3}\right) .{ }^{1} \mathbf{H} \mathbf{~ N M R}(400 \mathrm{MHz}$, Chloroform- $d$ ) $\delta 8.02(\mathrm{dd}, J=7.9,1.4 \mathrm{~Hz}, 1 \mathrm{H}), 7.44(\mathrm{td}, J=7.5,1.5 \mathrm{~Hz}, 1 \mathrm{H}), 7.29(\mathrm{td}, J=7.7,1.2 \mathrm{~Hz}, 1 \mathrm{H}), 7.22$ $(\mathrm{d}, J=7.6 \mathrm{~Hz}, 1 \mathrm{H}), 3.07-2.91(\mathrm{~m}, 2 \mathrm{H}), 2.54(\mathrm{ddt}, J=10.0,8.3,4.9 \mathrm{~Hz}, 1 \mathrm{H}), 2.30-2.16(\mathrm{~m}, 1 \mathrm{H}), 1.98-1.88(\mathrm{~m}$, 1H), $1.89-1.78(\mathrm{~m}, 1 \mathrm{H}), 1.79-1.54(\mathrm{~m}, 8 \mathrm{H}), 1.52-1.38(\mathrm{~m}, 4 \mathrm{H}), 1.34-1.10(\mathrm{~m}, 4 \mathrm{H}) .{ }^{13} \mathrm{C}$ NMR $(126 \mathrm{MHz}$, $\left.\mathrm{CDCl}_{3}\right) \delta 201.10,143.97,133.15,132.67,128.74,127.57,126.63,45.32,37.59,36.49,35.54,33.71,28.64,28.50$, 28.27, 26.54, 26.38. HRMS (ESI) $\mathrm{m} / \mathrm{z}(\mathrm{M}+\mathrm{H})^{+}$calculated for $\mathrm{C}_{18} \mathrm{H}_{27} \mathrm{O}: 271.2062$, observed: 271.2059. The absolute configuration was tentatively assigned by analogy.

(S)-2-phenethyl-3,4-dihydronaphthalen-1(2H)-one (25). Colorless oil. Yield: 58\%. Enantiomeric excess: 85\%, determined by HPLC (CHIRALPAK IG, hexane/ isopropanol $=97 / 3$, flow rate $1.0 \mathrm{~mL} / \mathrm{min}, \mathrm{T}=30^{\circ} \mathrm{C}, 254 \mathrm{~nm}$ ): $\mathrm{t}_{\mathrm{R}}=14.331 \min$ (minor), $\mathrm{t}_{\mathrm{R}}=13.409 \min$ (major). $[\alpha]_{\mathrm{D}}^{20}=23.8\left(\mathrm{c} 0.27 \mathrm{CHCl}_{3}\right) .{ }^{1} \mathbf{H} \mathbf{~ N M R}(400 \mathrm{MHz}$, Chloroform- $d$ ) $\delta 8.03(\mathrm{dd}, J=7.9,1.4 \mathrm{~Hz}, 1 \mathrm{H}), 7.45(\mathrm{td}, J=7.5,1.5 \mathrm{~Hz}, 1 \mathrm{H}), 7.35-7.09(\mathrm{~m}, 7 \mathrm{H}), 3.08-2.90(\mathrm{~m}$, 2H), $2.86-2.66(\mathrm{~m}, 2 \mathrm{H}), 2.51(\mathrm{dddd}, J=11.4,7.2,5.5,4.3 \mathrm{~Hz}, 1 \mathrm{H}), 2.40-2.22(\mathrm{~m}, 2 \mathrm{H}), 1.94$ (dddd, $J=13.2$, $11.2,9.2,5.8 \mathrm{~Hz}, 1 \mathrm{H}), 1.79$ (dddd, $J=13.3,9.6,7.2,5.8 \mathrm{~Hz}, 1 \mathrm{H}) .{ }^{13} \mathbf{C ~ N M R}\left(126 \mathrm{MHz}, \mathrm{CDCl}_{3}\right) \delta 200.29,144.00$, 142.16, 133.29, 132.69, 128.81, 128.62, 128.52, 127.58, 126.73, 126.00, 46.94, 33.30, 31.48, 28.65, 28.60. HRMS (ESI) $\mathrm{m} / \mathrm{z}(\mathrm{M}+\mathrm{H})^{+}$calculated for $\mathrm{C}_{18} \mathrm{H}_{19} \mathrm{O}: 251.1436$, observed: 251.1429. The absolute configuration was tentatively assigned by analogy

(S)-2-(2-methylphenethyl)-3,4-dihydronaphthalen-1(2H)-one (26). Colorless oil. Yield: 43\%. Enantiomeric excess: 93\%, determined by HPLC (CHIRALPAK IC, hexane/ isopropanol $=97 / 3$, flow rate $1.0 \mathrm{~mL} / \mathrm{min}, \mathrm{T}=$ $30^{\circ} \mathrm{C}, 254 \mathrm{~nm}$ ): $\mathrm{t}_{\mathrm{R}}=7.980 \mathrm{~min}$ (minor), $\mathrm{t}_{\mathrm{R}}=8.410 \mathrm{~min}$ (major). $[\alpha]_{\mathrm{D}}{ }^{20}=18.8\left(\mathrm{c} 0.27 \mathrm{CHCl}_{3}\right) .{ }^{1} \mathbf{H ~} \mathbf{~ N M R}(400 \mathrm{MHz}$, Chloroform- $d$ ) $\delta 8.05(\mathrm{dd}, J=7.9,1.4 \mathrm{~Hz}, 1 \mathrm{H}), 7.47(\mathrm{td}, J=7.5,1.5 \mathrm{~Hz}, 1 \mathrm{H}), 7.31(\mathrm{t}, J=7.8 \mathrm{~Hz}, 1 \mathrm{H}), 7.25(\mathrm{~d}, J=$ $7.5 \mathrm{~Hz}, 1 \mathrm{H}), 7.21-7.17(\mathrm{~m}, 1 \mathrm{H}), 7.17-7.08(\mathrm{~m}, 3 \mathrm{H}), 3.04$ (dd, $J=7.5,4.8 \mathrm{~Hz}, 2 \mathrm{H}), 2.75$ (ddd, $J=9.3,6.5,2.5$ $\mathrm{Hz}, 2 \mathrm{H}), 2.64-2.50(\mathrm{~m}, 1 \mathrm{H}), 2.36$ (s, 3H), $2.36-2.14(\mathrm{~m}, 2 \mathrm{H}), 2.01$ (ddt, $J=13.3,11.3,7.5 \mathrm{~Hz}, 1 \mathrm{H}), 1.78$ (ddt, $J$ $=13.5,9.8,6.7 \mathrm{~Hz}, 1 \mathrm{H}) .{ }^{13} \mathbf{C} \mathbf{N M R}\left(126 \mathrm{MHz}, \mathrm{CDCl}_{3}\right) \delta 200.25,144.00,140.48,136.15,133.30,132.73,130.36$, $129.01,128.83,127.58,126.75,126.14,126.10,47.39,30.78,30.43,28.82,28.70,19.45$. HRMS (ESI) m/z $(\mathrm{M}+\mathrm{H})^{+}$calculated for $\mathrm{C}_{19} \mathrm{H}_{21} \mathrm{O}: 265.1592$, observed: 265.1587. The absolute configuration was tentatively assigned by analogy

(S)-2-(3-methylphenethyl)-3,4-dihydronaphthalen-1(2H)-one (27). Colorless oil. Yield: 40\%. Enantiomeric excess: $86 \%$, determined by HPLC (CHIRALPAK OJ, hexane $/$ isopropanol $=70 / 30$, flow rate $1.0 \mathrm{~mL} / \mathrm{min}, \mathrm{T}=$ $30^{\circ} \mathrm{C}, 254 \mathrm{~nm}$ ): $\mathrm{t}_{\mathrm{R}}=5.288 \mathrm{~min}$ (minor), $\mathrm{t}_{\mathrm{R}}=5.755 \mathrm{~min}$ (major). $[\alpha]_{\mathrm{D}}{ }^{20}=15.9\left(\mathrm{c} 1.2, \mathrm{CHCl}_{3}\right) .{ }^{1} \mathbf{H} \mathbf{~ N M R}(400 \mathrm{MHz}$, Chloroform- $d$ ) $\delta 8.04(\mathrm{dd}, J=7.9,1.4 \mathrm{~Hz}, 1 \mathrm{H}), 7.46(\mathrm{td}, J=7.5,1.5 \mathrm{~Hz}, 1 \mathrm{H}), 7.35-7.26(\mathrm{~m}, 1 \mathrm{H}), 7.24(\mathrm{~d}, J=7.7$ $\mathrm{Hz}, 1 \mathrm{H}), 7.18(\mathrm{t}, J=7.5 \mathrm{~Hz}, 1 \mathrm{H}), 7.07(\mathrm{~s}, 1 \mathrm{H}), 7.04(\mathrm{~d}, J=7.6 \mathrm{~Hz}, 1 \mathrm{H}), 7.01(\mathrm{~d}, J=7.6 \mathrm{~Hz}, 1 \mathrm{H}), 3.06-2.93(\mathrm{~m}$, 
2H), $2.82-2.64(\mathrm{~m}, 2 \mathrm{H}), 2.52$ (dddd, $J=11.3,7.2,5.5,4.3 \mathrm{~Hz}, 2 \mathrm{H}), 2.33(\mathrm{~s}, 3 \mathrm{H}), 2.37-2.24(\mathrm{~m}, 2 \mathrm{H}), 1.95$ (dddd, $J=13.3,11.1,9.2,5.8 \mathrm{~Hz}, 1 \mathrm{H}), 1.79(\mathrm{dddd}, J=13.3,9.7,7.2,5.8 \mathrm{~Hz}, 1 \mathrm{H}) .{ }^{13} \mathbf{C} \mathbf{N M R}\left(126 \mathrm{MHz}, \mathrm{CDCl}_{3}\right) \delta 200.31$, 144.00, 142.08, 138.05, 133.26, 132.68, 129.42, 128.80, 128.38, 127.56, 126.73, 126.70, 125.60, 46.95, 33.20, 31.49, 28.60, 28.57, 21.54. HRMS (ESI) $\mathrm{m} / \mathrm{z}(\mathrm{M}+\mathrm{H})^{+}$calculated for $\mathrm{C}_{19} \mathrm{H}_{21} \mathrm{O}: 265.1592$, observed: 265.1583. The absolute configuration was tentatively assigned by analogy

(S)-2-(3,5-dimethylphenethyl)-3,4-dihydronaphthalen-1(2H)-one (28) Colorless oil. Yield: 61\%. Enantiomeric excess: $81 \%$, determined by HPLC (CHIRALPAK ID, hexane/ isopropanol $=94 / 6$, flow rate $1.0 \mathrm{~mL} / \mathrm{min}, \mathrm{T}=$ $30^{\circ} \mathrm{C}, 240 \mathrm{~nm}$ ): $\mathrm{t}_{\mathrm{R}}=6.594 \mathrm{~min}$ (minor), $\mathrm{t}_{\mathrm{R}}=6.255 \mathrm{~min}$ (major). $[\alpha]_{\mathrm{D}}{ }^{20}=20.3\left(\mathrm{c} 1.6, \mathrm{CHCl}_{3}\right) .{ }^{1} \mathbf{H} \mathbf{~ N M R}(400 \mathrm{MHz}$, Chloroform- $d$ ) $\delta 8.05(\mathrm{dd}, J=7.9,1.4 \mathrm{~Hz}, 1 \mathrm{H}), 7.47(\mathrm{td}, J=7.5,1.5 \mathrm{~Hz}, 1 \mathrm{H}), 7.36-7.27(\mathrm{~m}, 1 \mathrm{H}), 7.24(\mathrm{~d}, J=7.7$ $\mathrm{Hz}, 1 \mathrm{H}), 6.88(\mathrm{~s}, 2 \mathrm{H}), 6.84(\mathrm{~s}, 1 \mathrm{H}), 3.07-2.95(\mathrm{~m}, 2 \mathrm{H}), 2.78-2.62(\mathrm{~m}, 2 \mathrm{H}), 2.53$ (dddd, $J=11.3,7.2,5.5,4.4 \mathrm{~Hz}$, $1 \mathrm{H}), 2.36-2.25(\mathrm{~m}, 2 \mathrm{H}), 2.30(\mathrm{~s}, 6 \mathrm{H}), 1.96(\mathrm{dddd}, J=13.3,11.1,9.2,5.7 \mathrm{~Hz}, 1 \mathrm{H}), 1.79$ (dddd, $J=13.2,9.8,7.2$, $5.7 \mathrm{~Hz}, 1 \mathrm{H}) .{ }^{13} \mathrm{C}$ NMR $\left(126 \mathrm{MHz}, \mathrm{CDCl}_{3}\right) \delta 200.33,144.01,142.07,137.95,133.24,132.69,128.80,127.62$, 127.56, 126.70, 126.44, 47.00, 33.11, 31.52, 28.58, 28.55, 21.41. HRMS (ESI) m/z (M+H) ${ }^{+}$calculated for $\mathrm{C}_{20} \mathrm{H}_{23} \mathrm{O}: 279.1749$, observed: 279.1739 . The absolute configuration was tentatively assigned by analogy

(S)-2-(4-methylphenethyl)-3,4-dihydronaphthalen-1(2H)-one (29) Colorless oil. Yield: 44\%. Enantiomeric excess: $85 \%$, determined by HPLC (CHIRALPAK IG, hexane/ isopropanol $=94 / 6$, flow rate $1.0 \mathrm{~mL} / \mathrm{min}, \mathrm{T}=$ $30^{\circ} \mathrm{C}, 240 \mathrm{~nm}$ ): $\mathrm{t}_{\mathrm{R}}=10.320 \mathrm{~min}$ (minor), $\mathrm{t}_{\mathrm{R}}=10.906 \mathrm{~min}$ (major). $[\alpha]_{\mathrm{D}}{ }^{20}=29.4\left(\mathrm{c} \mathrm{0.67}, \mathrm{CHCl}_{3}\right) .{ }^{1} \mathbf{H} \mathbf{~ N M R}(400$ MHz, Chloroform- $d$ ) $\delta 8.04(\mathrm{dd}, J=7.8,1.4 \mathrm{~Hz}, 1 \mathrm{H}), 7.46(\mathrm{td}, J=7.4,1.5 \mathrm{~Hz}, 1 \mathrm{H}), 7.31(\mathrm{t}, J=7.5 \mathrm{~Hz}, 1 \mathrm{H}), 7.23$ $(\mathrm{d}, J=7.7 \mathrm{~Hz}, 1 \mathrm{H}), 7.12(\mathrm{q}, J=8.0 \mathrm{~Hz}, 4 \mathrm{H}), 3.06-2.93(\mathrm{~m}, 2 \mathrm{H}), 2.73$ (qdd, $J=13.7,9.7,6.2 \mathrm{~Hz}, 2 \mathrm{H}), 2.51$ (ddt, $J=11.2,7.0,4.7 \mathrm{~Hz}, 1 \mathrm{H}), 2.32(\mathrm{~s}, 3 \mathrm{H}), 2.38-2.24(\mathrm{~m}, 2 \mathrm{H}), 1.95$ (dddd, $J=13.2,11.0,9.2,5.7 \mathrm{~Hz}, 1 \mathrm{H}), 1.78$ (dddd, $J=13.3,9.7,7.2,5.7 \mathrm{~Hz}, 1 \mathrm{H}) .{ }^{13} \mathbf{C}$ NMR $\left(126 \mathrm{MHz}, \mathrm{CDCl}_{3}\right) \delta 200.34,144.00,139.02,135.42,133.25$, $132.69,129.19,128.80,128.49,127.56,126.70,46.88,32.81,31.53,28.60,28.56,21.15$. HRMS (ESI) m/z $(\mathrm{M}+\mathrm{H})^{+}$calculated for $\mathrm{C}_{19} \mathrm{H}_{21} \mathrm{O}: 265.1592$, observed: 265.1583 . The absolute configuration was tentatively assigned by analogy

(S)-2-(4-fluorophenethyl)-3,4-dihydronaphthalen-1(2H)-one (30) Colorless oil. Yield: 36\%. Enantiomeric excess: $84 \%$, determined by HPLC (CHIRALPAK AS-H, hexane $/$ isopropanol $=90 / 10$, flow rate $1.0 \mathrm{~mL} / \mathrm{min}, \mathrm{T}=$ $30^{\circ} \mathrm{C}, 254 \mathrm{~nm}$ ): $\mathrm{t}_{\mathrm{R}}=5.530 \mathrm{~min}$ (minor), $\mathrm{t}_{\mathrm{R}}=6.017 \mathrm{~min}$ (major). $[\alpha]_{\mathrm{D}}{ }^{20}=20.6\left(\mathrm{c} 1.1, \mathrm{CHCl}_{3}\right) .{ }^{1} \mathbf{H} \mathbf{~ N M R}(400 \mathrm{MHz}$, Chloroform- $d$ ) $\delta 8.03(\mathrm{dd}, J=7.8,1.4 \mathrm{~Hz}, 1 \mathrm{H}), 7.46(\mathrm{td}, J=7.5,1.5 \mathrm{~Hz}, 1 \mathrm{H}), 7.34-7.28(\mathrm{~m}, 1 \mathrm{H}), 7.23(\mathrm{~d}, J=7.7$ $\mathrm{Hz}, 1 \mathrm{H}), 7.21-7.16(\mathrm{~m}, 2 \mathrm{H}), 7.01-6.93(\mathrm{~m}, 2 \mathrm{H}), 3.08-2.93(\mathrm{~m}, 2 \mathrm{H}), 2.81-2.63$ (m, 2H), 2.50 (dddd, $J=11.3$, 7.0, 5.6, 4.4 Hz, 1H), $2.38-2.21(\mathrm{~m}, 2 \mathrm{H}), 2.02-1.86(\mathrm{~m}, 1 \mathrm{H}), 1.84-1.71(\mathrm{~m}, 1 \mathrm{H}) .{ }^{13} \mathbf{C ~ N M R}\left(101 \mathrm{MHz}, \mathrm{CDCl}_{3}\right)$ $\delta 200.19,161.41(\mathrm{~d}, J=243.4 \mathrm{~Hz}), 143.97,137.75(\mathrm{~d}, J=3.1 \mathrm{~Hz}), 133.34,132.64,129.91(\mathrm{~d}, J=7.8 \mathrm{~Hz}), 128.83$, 127.57, 126.76, $115.22(\mathrm{~d}, J=21.0 \mathrm{~Hz}), 46.84,32.49,31.67,28.70,28.62$. HRMS (ESI) m/z $(\mathrm{M}+\mathrm{H})^{+}$calculated for $\mathrm{C}_{18} \mathrm{H}_{18} \mathrm{OF}$ : 269.1342, observed: 269.1347 . The absolute configuration was tentatively assigned by analogy

(S)-2-(4-chlorophenethyl)-3,4-dihydronaphthalen-1(2H)-one (31) Colorless oil. Yield: 44\%. Enantiomeric excess: $86 \%$, determined by HPLC (CHIRALPAK ID, hexane/ isopropanol = 97/3, flow rate $1.0 \mathrm{~mL} / \mathrm{min}, \mathrm{T}=$ $30^{\circ} \mathrm{C}, 240 \mathrm{~nm}$ ): $\mathrm{t}_{\mathrm{R}}=10.501 \mathrm{~min}$ (minor), $\mathrm{t}_{\mathrm{R}}=9.945 \min$ (major). $[\alpha]_{\mathrm{D}}{ }^{20}=22.5$ (c 1.3, $\left.\mathrm{CHCl}_{3}\right) .{ }^{1} \mathbf{H} \mathbf{~ N M R}(400 \mathrm{MHz}$, Chloroform- $d$ ) $\delta 8.05(\mathrm{dd}, J=7.8,1.4 \mathrm{~Hz}, 1 \mathrm{H}), 7.49(\mathrm{td}, J=7.5,1.5 \mathrm{~Hz}, 1 \mathrm{H}), 7.33(\mathrm{td}, J=7.6,1.2 \mathrm{~Hz}, 1 \mathrm{H}), 7.29-$ $7.24(\mathrm{~m}, 3 \mathrm{H}), 7.22-7.17(\mathrm{~m}, 2 \mathrm{H}), 3.09-2.93(\mathrm{~m}, 2 \mathrm{H}), 2.87-2.65(\mathrm{~m}, 2 \mathrm{H}), 2.51$ (dddd, $J=11.3,6.9,5.7,4.4 \mathrm{~Hz}$, $1 \mathrm{H}), 2.36-2.22(\mathrm{~m}, 2 \mathrm{H}), 2.04-1.90(\mathrm{~m}, 1 \mathrm{H}), 1.86-1.70(\mathrm{~m}, 1 \mathrm{H}) .{ }^{13} \mathbf{C} \mathbf{N M R}\left(101 \mathrm{MHz}, \mathrm{CDCl}_{3}\right) \delta 200.12$, 
143.95, 140.60, 133.36, 132.62, 131.69, 129.96, 128.83, 128.59, 127.56, 126.76, 46.82, 32.65, 31.47, 28.73, 28.63. HRMS (ESI) $\mathrm{m} / \mathrm{z}(\mathrm{M}+\mathrm{H})^{+}$calculated for $\mathrm{C}_{18} \mathrm{H}_{18} \mathrm{OCl}$ : 285.1046, observed: 285.1044. The absolute configuration was tentatively assigned by analogy

(S)-2-(3,4-dimethylpent-3-en-1-yl)-3,4-dihydronaphthalen-1(2H)-one (32) Colorless oil. Yield: $52 \%$. Enantiomeric excess: 93\%, determined by HPLC (CHIRALPAK IC, hexane/ isopropanol $=99 / 1$, flow rate 1.0 $\mathrm{mL} / \mathrm{min}, \mathrm{T}=30^{\circ} \mathrm{C}, 254 \mathrm{~nm}$ ): $\mathrm{t}_{\mathrm{R}}=6.642 \mathrm{~min}$ (minor), $\mathrm{t}_{\mathrm{R}}=7.118 \mathrm{~min}$ (major). $[\alpha]_{\mathrm{D}}{ }^{20}=20.0\left(\mathrm{c} 0.63, \mathrm{CHCl}_{3}\right) .{ }^{1} \mathbf{H}$ NMR (400 MHz, Chloroform- $d$ ) $\delta 8.03(\mathrm{dd}, J=7.9,1.4 \mathrm{~Hz}, 1 \mathrm{H}), 7.44(\mathrm{dd}, J=7.5,1.5 \mathrm{~Hz}, 1 \mathrm{H}), 7.29(\mathrm{t}, J=7.6$ $\mathrm{Hz}, 1 \mathrm{H}), 7.23(\mathrm{~d}, J=7.6 \mathrm{~Hz}, 1 \mathrm{H}), 3.07-2.93(\mathrm{~m}, 2 \mathrm{H}), 2.44(\mathrm{ddt}, J=11.7,7.4,4.7 \mathrm{~Hz}, 1 \mathrm{H}), 2.26(\mathrm{dq}, J=13.8,4.7$ $\mathrm{Hz}, 1 \mathrm{H}), 2.18-2.10(\mathrm{~m}, 2 \mathrm{H}), 2.06-1.88(\mathrm{~m}, 2 \mathrm{H}), 1.67$ (s, 6H), $1.64(\mathrm{~s}, 3 \mathrm{H}), 1.59-1.44(\mathrm{~m}, 1 \mathrm{H}) .{ }^{13} \mathbf{C}$ NMR $(101$ $\left.\mathrm{MHz}, \mathrm{CDCl}_{3}\right) \delta 200.54,144.03,133.17,132.74,128.78,127.52,127.47,126.66,124.66,47.42,31.91,28.62$, 28.52, 28.04, 20.72, 20.28, 18.47. HRMS (ESI) $\mathrm{m} / \mathrm{z}(\mathrm{M}+\mathrm{H})^{+}$calculated for $\mathrm{C}_{17} \mathrm{H}_{23} \mathrm{O}: 243.1749$, observed: 243.1740. The absolute configuration was tentatively assigned by analogy.

(R)-2-(2-oxobutyl)-3,4-dihydronaphthalen-1(2H)-one (33) Colorless oil. Yield: 51\%. Enantiomeric excess: $61 \%$, determined by HPLC (CHIRALPAK IE, hexane/ isopropanol $=94 / 6$, flow rate $1.0 \mathrm{~mL} / \mathrm{min}, \mathrm{T}=30^{\circ} \mathrm{C}, 254$ $\mathrm{nm}): \mathrm{t}_{\mathrm{R}}=24.870 \mathrm{~min}$ (minor), $\mathrm{t}_{\mathrm{R}}=23.543 \min$ (major). $[\alpha]_{\mathrm{D}}{ }^{20}=-11.6\left(\mathrm{c} 0.5, \mathrm{CHCl}_{3}\right) .{ }^{1} \mathbf{H} \mathbf{~ N M R}(500 \mathrm{MHz}$, Chloroform- $d$ ) $\delta 7.99(\mathrm{dd}, J=7.9,1.4 \mathrm{~Hz}, 1 \mathrm{H}), 7.46(\mathrm{td}, J=7.5,1.5 \mathrm{~Hz}, 1 \mathrm{H}), 7.29(\mathrm{t}, J=7.6 \mathrm{~Hz}, 1 \mathrm{H}), 7.23(\mathrm{~d}, J=$ $7.7 \mathrm{~Hz}, 1 \mathrm{H}), 3.21-3.07(\mathrm{~m}, 3 \mathrm{H}), 2.95(\mathrm{ddd}, J=16.8,4.3,2.8 \mathrm{~Hz}, 1 \mathrm{H}), 2.64(\mathrm{dq}, J=17.8,7.3 \mathrm{~Hz}, 1 \mathrm{H}), 2.51$ (dq, $J$ $=17.7,7.3 \mathrm{~Hz}, 1 \mathrm{H}), 2.46-2.38(\mathrm{~m}, 1 \mathrm{H}), 2.20-2.13(\mathrm{~m}, 1 \mathrm{H}), 1.92(\mathrm{qd}, J=13.1,4.3 \mathrm{~Hz}, 1 \mathrm{H}), 1.10(\mathrm{t}, J=7.3 \mathrm{~Hz}$, 3H). ${ }^{13} \mathbf{C}$ NMR $\left(126 \mathrm{MHz}, \mathrm{CDCl}_{3}\right) \delta 210.10,199.36,144.23,133.49,132.29,128.87,127.47,126.71,44.35,42.78$, 36.60, 29.65, 29.53, 7.95. HRMS (ESI) $\mathrm{m} / \mathrm{z}(\mathrm{M}+\mathrm{H})^{+}$calculated for $\mathrm{C}_{14} \mathrm{H}_{16} \mathrm{O}_{2}: 216.1150$, observed: 216.1150. The absolute configuration was tentatively assigned by analogy.

\section{Some unsuccessful product}

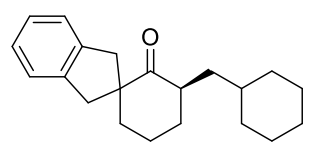

complex

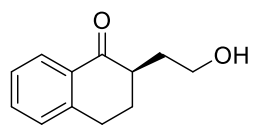

$31 \%$ yield, $0 \%$ ee

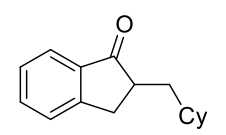

$67 \%$ yield, $0 \%$ ee

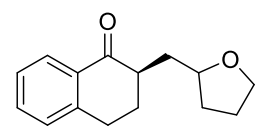

$52 \%$ yield, $4 \%$ ee

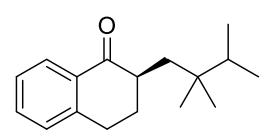

$28 \%$ yield, $76 \%$ ee<smiles>CN(CC[C@H]1CCc2ccccc2C1=O)c1ccccc1</smiles>

$12 \%$ yield, $0 \%$ ee

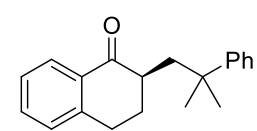

$47 \%$ yield, $57 \%$ ee

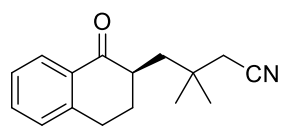

$64 \%$ yield, $4 \%$ ee

\section{X-ray Single Crystal - Data of 6 (CCDC 1978529)}

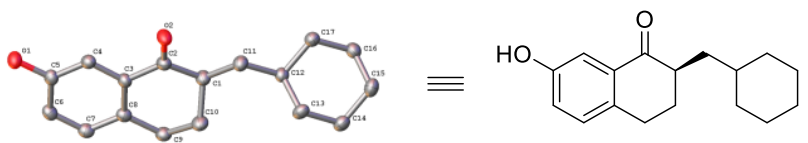

Crystal data and structure refinement for $\mathbf{6}$

\begin{tabular}{ll}
\hline Identification code & 191220dzy_0m \\
\hline Empirical formula & C17 H22 O2 \\
\hline Formula weight & 258.34 \\
\hline Temperature/K & $170.0 \mathrm{~K}$
\end{tabular}




\begin{tabular}{|c|c|}
\hline Wavelength & $1.34139 \AA$ \\
\hline Crystal system & Monoclinic \\
\hline Space group & P 1211 \\
\hline \multirow[t]{3}{*}{ Unit cell dimensions } & $\mathrm{a}=15.2490(8) \AA=90^{\circ}$. \\
\hline & $\mathrm{b}=6.1775(3) \AA=113.939(2)^{\circ}$. \\
\hline & $\mathrm{c}=16.2653(9) \AA=90^{\circ}$. \\
\hline Volume $/ \AA^{3}$ & $1400.40(13) \AA^{3}$ \\
\hline $\mathrm{Z}$ & 4 \\
\hline Density (calculated) & $1.225 \mathrm{Mg} / \mathrm{m}^{3}$ \\
\hline Absorption coefficient & $0.395 \mathrm{~mm}^{-1}$ \\
\hline $\mathrm{F}(000)$ & 560 \\
\hline Crystal size & $0.1 \times 0.02 \times 0.006 \mathrm{~mm}^{3}$ \\
\hline Theta range for data collection & 2.758 to $55.037^{\circ}$. \\
\hline Index ranges & $-18<=\mathrm{h}<=18,-7<=\mathrm{k}<=7,-18<=1<=19$ \\
\hline Reflections collected & 15994 \\
\hline Independent reflections & $5138[\mathrm{R}(\mathrm{int})=0.0503]$ \\
\hline Completeness to theta $=53.594^{\circ}$ & $99.1 \%$ \\
\hline Absorption correction & Semi-empirical from equivalents \\
\hline Max. and min. transmission & 0.7508 and 0.4245 \\
\hline Refinement method & Full-matrix least-squares on $\mathrm{F}^{2}$ \\
\hline Data / restraints / parameters & $5138 / 1 / 346$ \\
\hline Goodness-of-fit on $\mathrm{F}^{2}$ & 1.025 \\
\hline Final $\mathrm{R}$ indices $[\mathrm{I}>2 \operatorname{sigma}(\mathrm{I})]$ & $\mathrm{R} 1=0.0652, \mathrm{wR} 2=0.1546$ \\
\hline $\mathrm{R}$ indices (all data) & $\mathrm{R} 1=0.0877, \mathrm{wR} 2=0.1739$ \\
\hline Absolute structure parameter & $0.20(18)$ \\
\hline Extinction coefficient & $0.0073(14)$ \\
\hline Largest diff. peak and hole & 0.248 and -0.248 e. $\AA^{-3}$ \\
\hline
\end{tabular}

\section{References}

1. (a)Xu, B.; Zhu, S.-F.; Xie, X.-L.; Shen, J.-J.; Zhou, Q.-L. Asymmetric N-H Insertion Reaction Cooperatively Catalyzed by Rhodium and Chiral Spiro Phosphoric Acids. Angew. Chem., Int. Ed. 2011, 50, 11483-11486. (b) Xu, B.; Zhu, S.-F.; Zhang, Z.-C; Yu, Z.-X.; Ma, Y.; Zhou, Q.-L. Highly Enantioselective S-H Insertion Reaction Cooperatively Catalyzed by Dirhodium Complexes and Chiral Spiro Phosphoric Acids. Chem. Sci. 2014, 5, 1442-1448.

2. Protti, S.; Ravelli, D.; Fagnoni, M.; Albini, A. Solar light-driven photocatalyzed alkylations. Chemistry on the window ledge Chem. Commun. 2009, 7351.

3. Pan, Y.; Qi L.-Z.; Zhou L.-B.; Li, M.-L.; Xue, X.-S.; Zhu, S.-F,; Zhou, Q.-L. Chiral Spiro Phosphoric Acid-Catalyzed Friedel-Crafts Conjugate Addition/Enantioselective Protonation Reactions. ACS Catal. 2019, $9,7,6522-6529$ 


\section{NMR spectra}
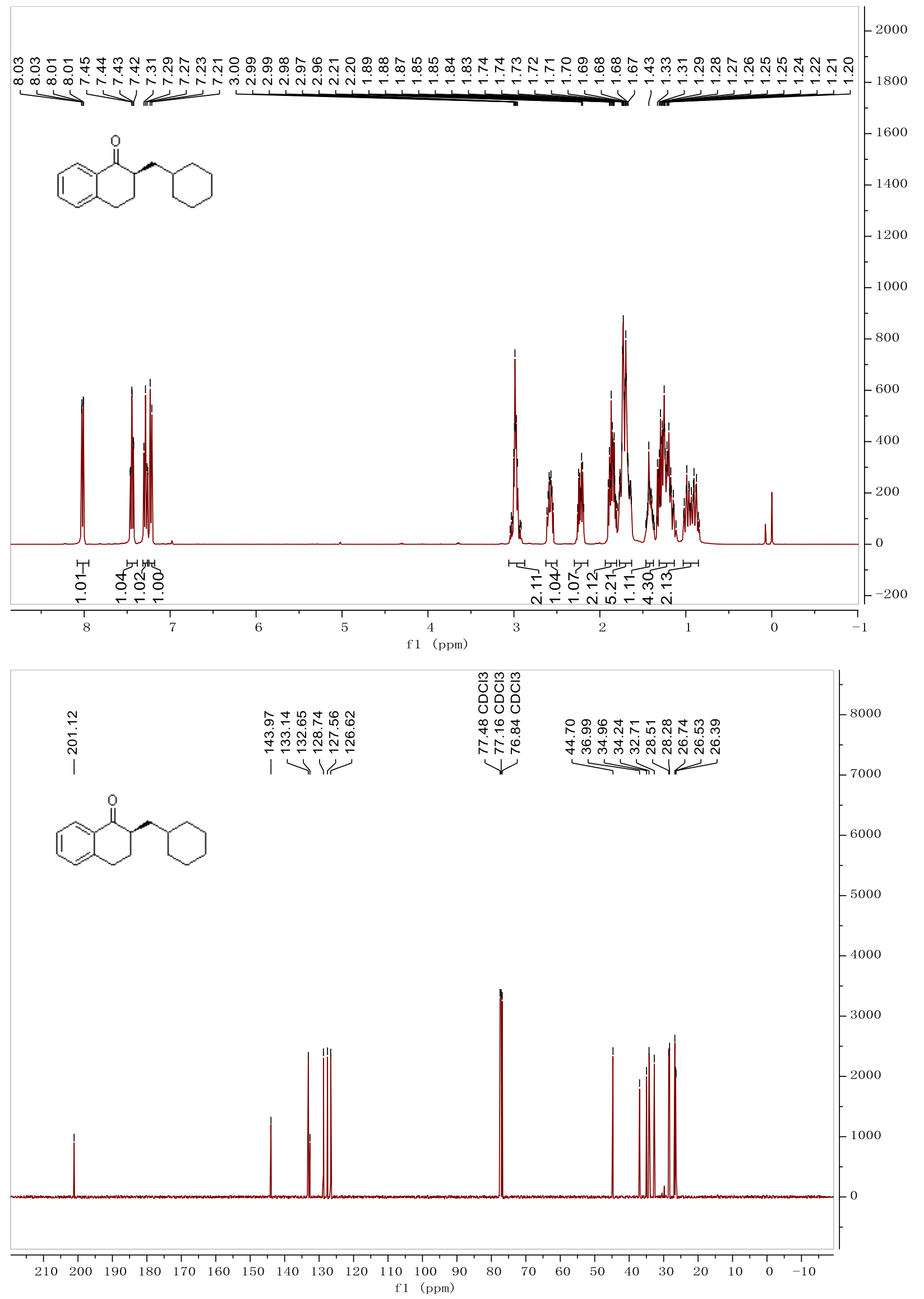

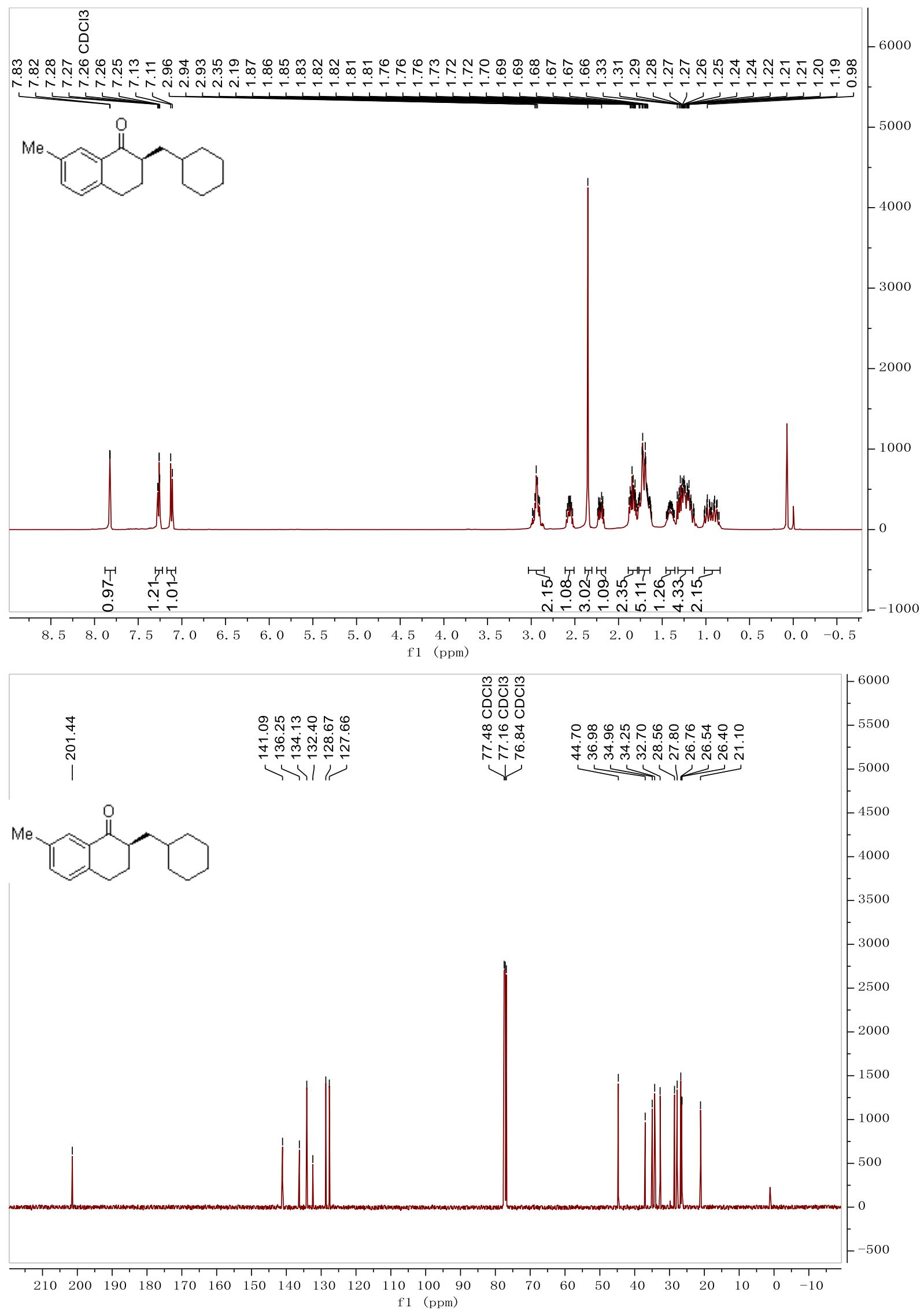

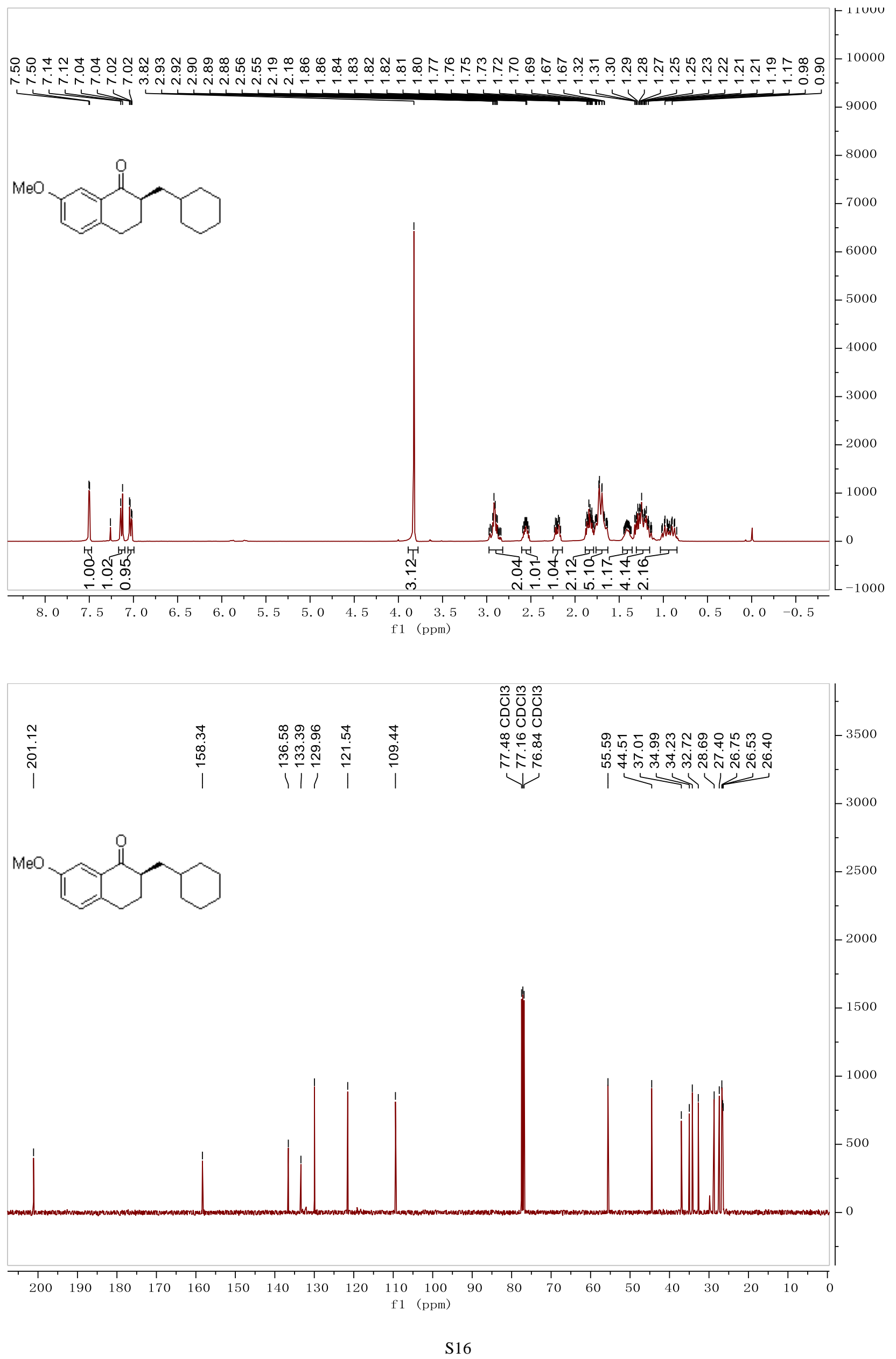


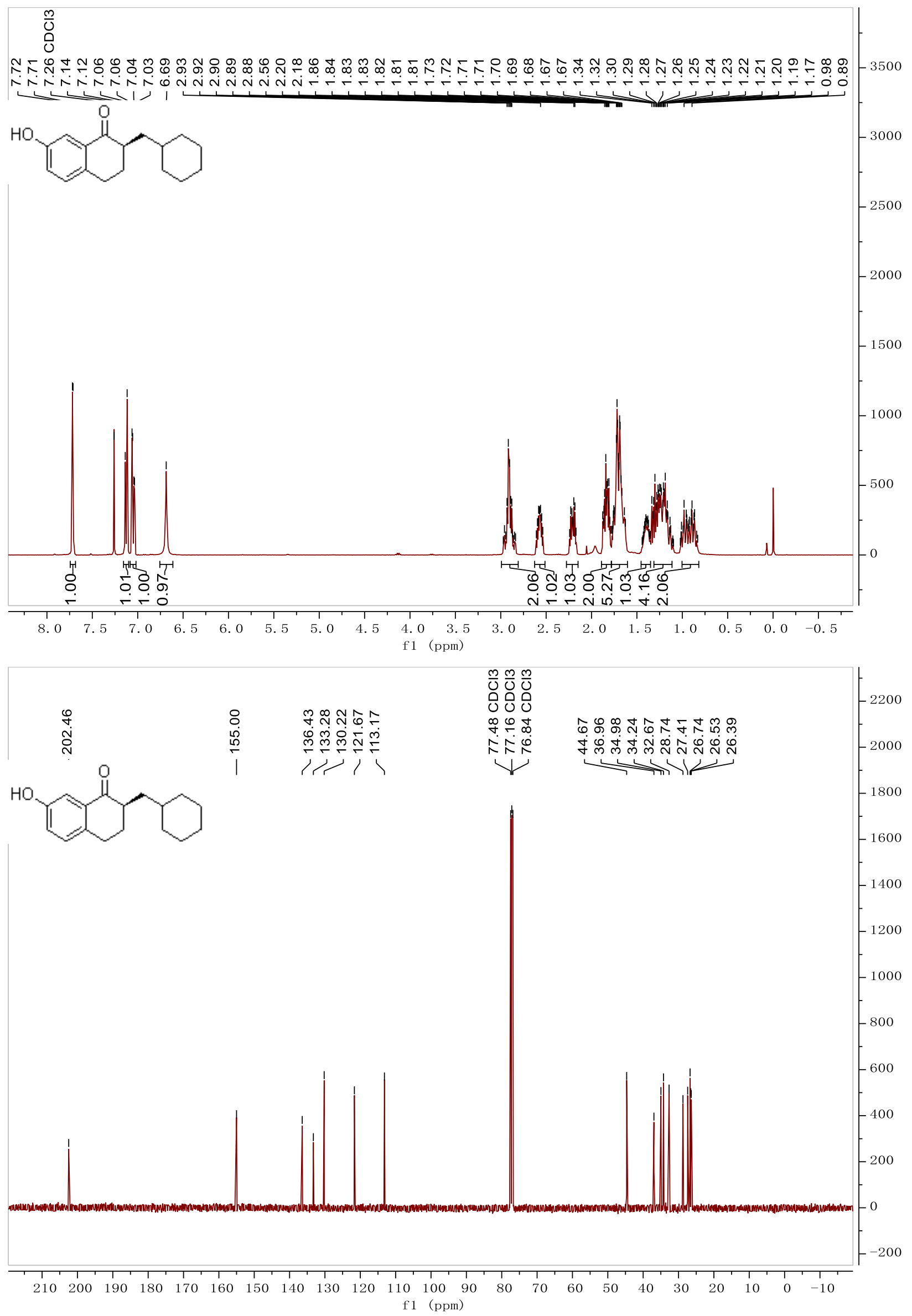



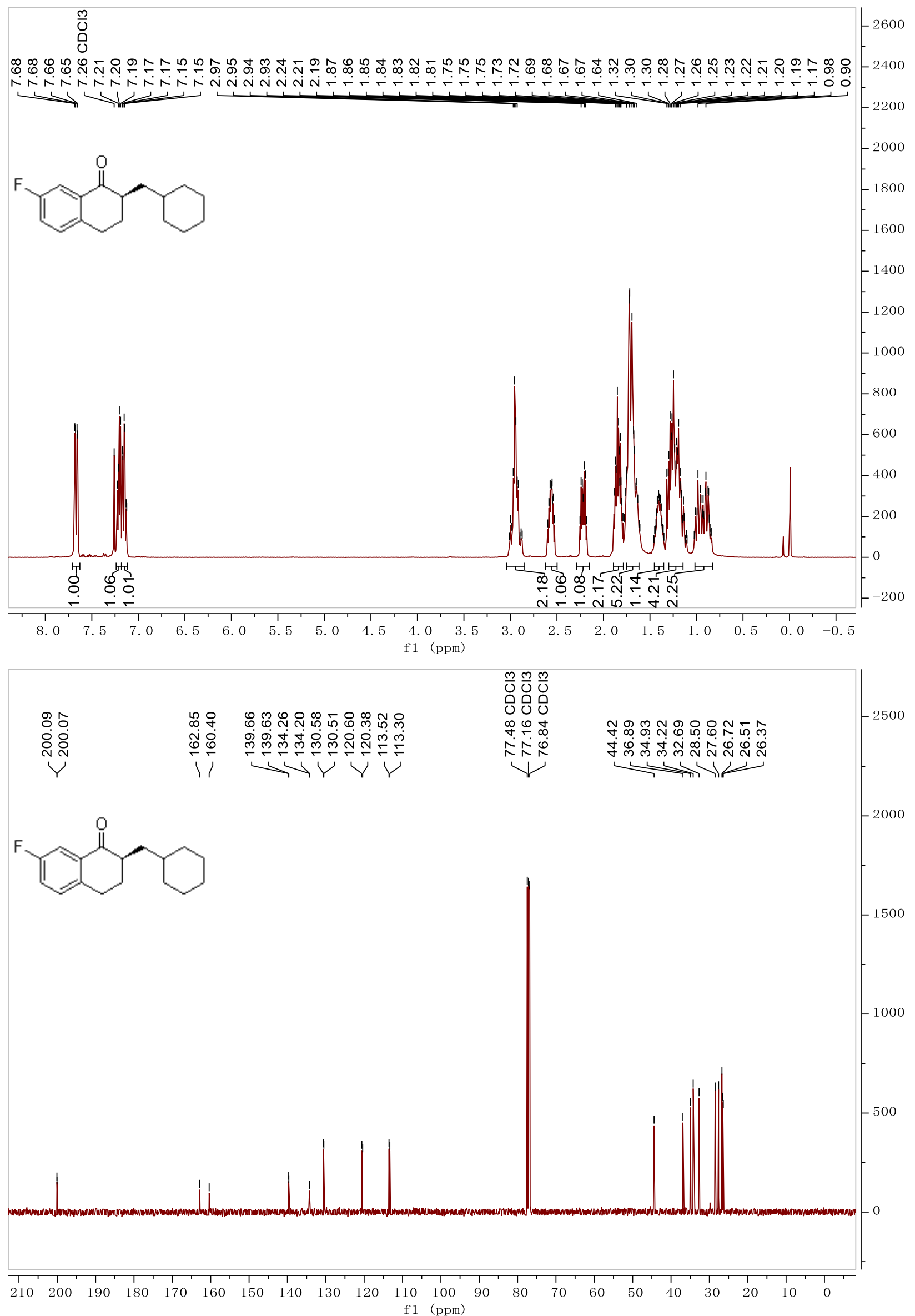


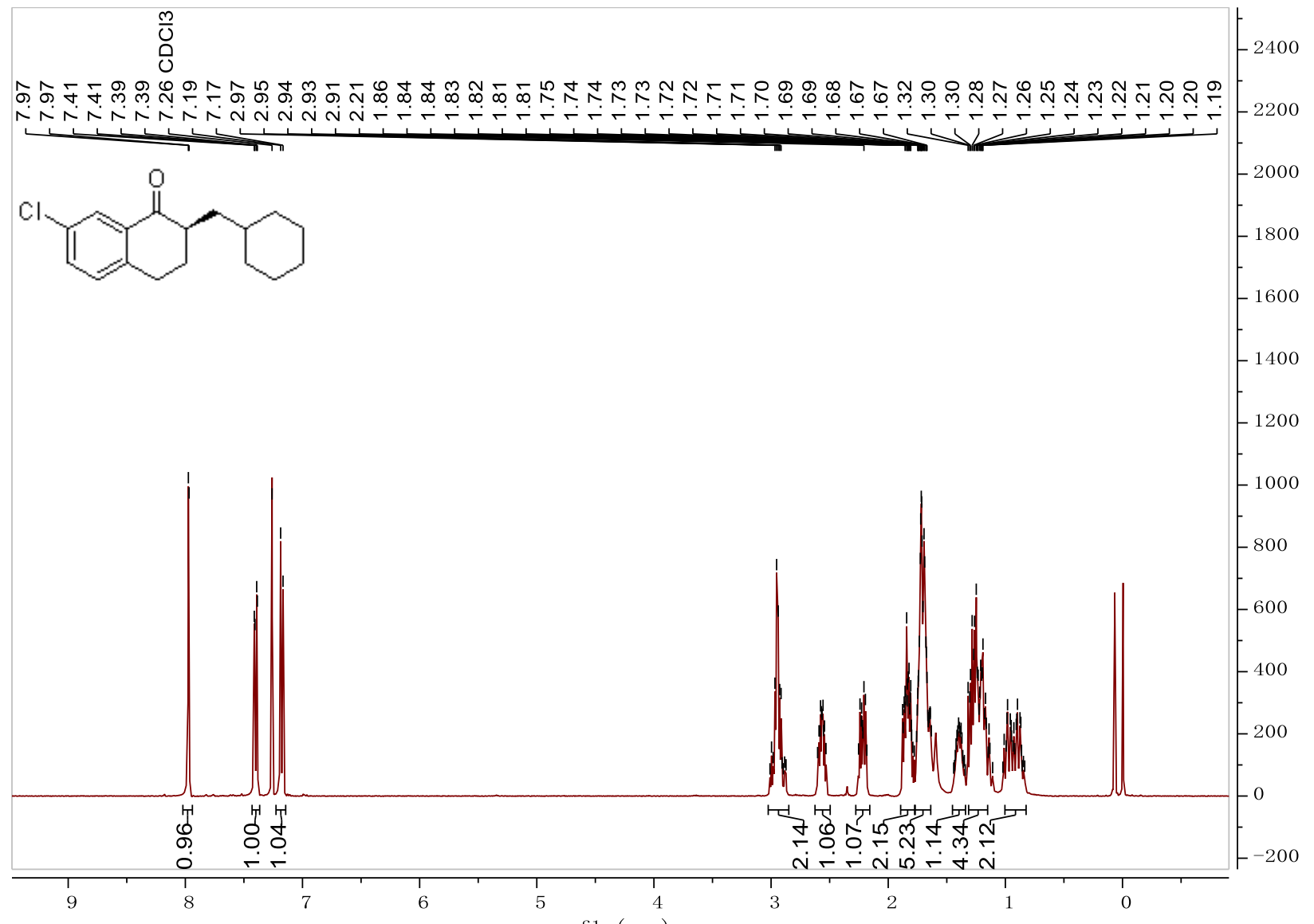

f1 (ppm)

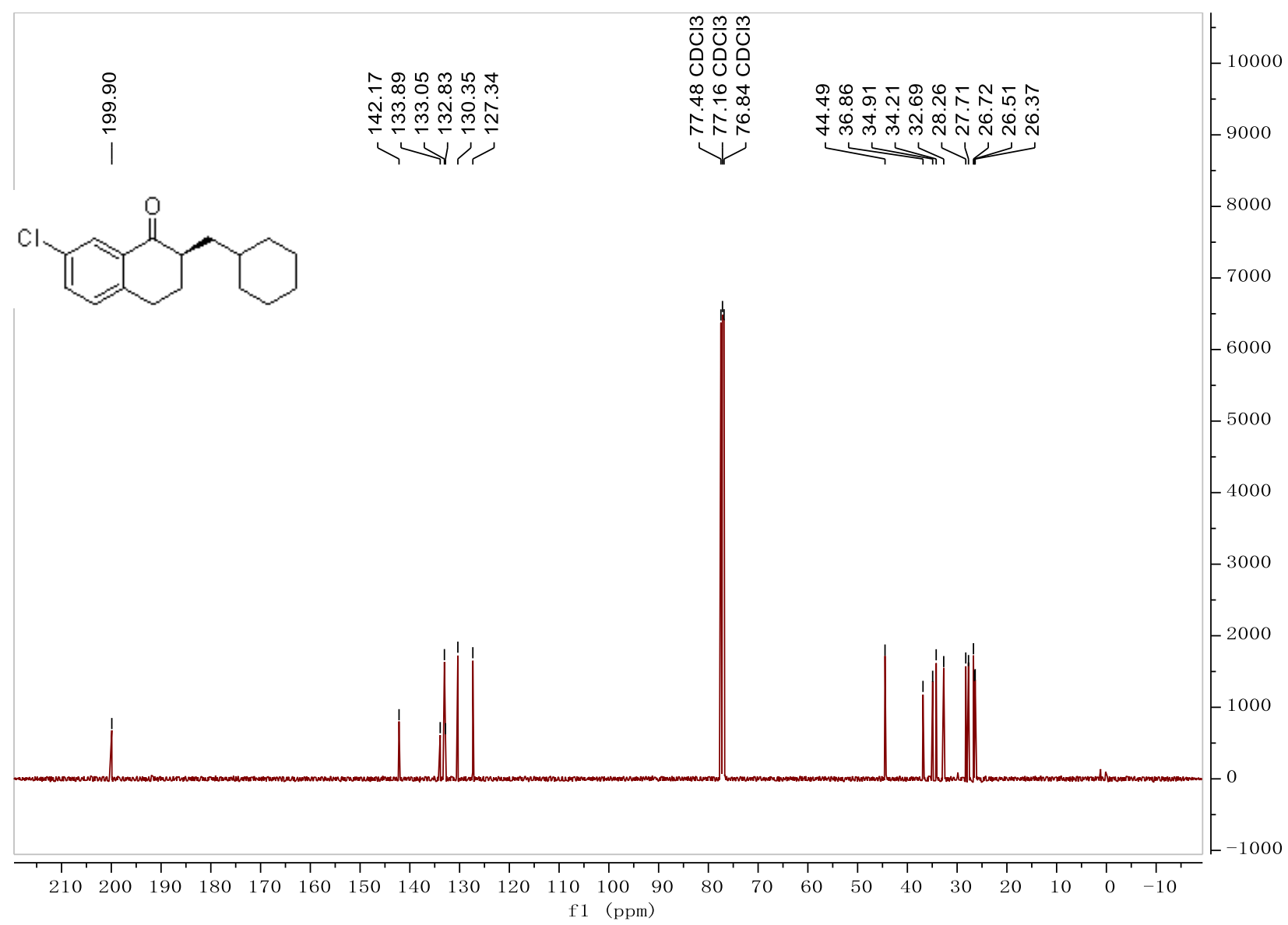




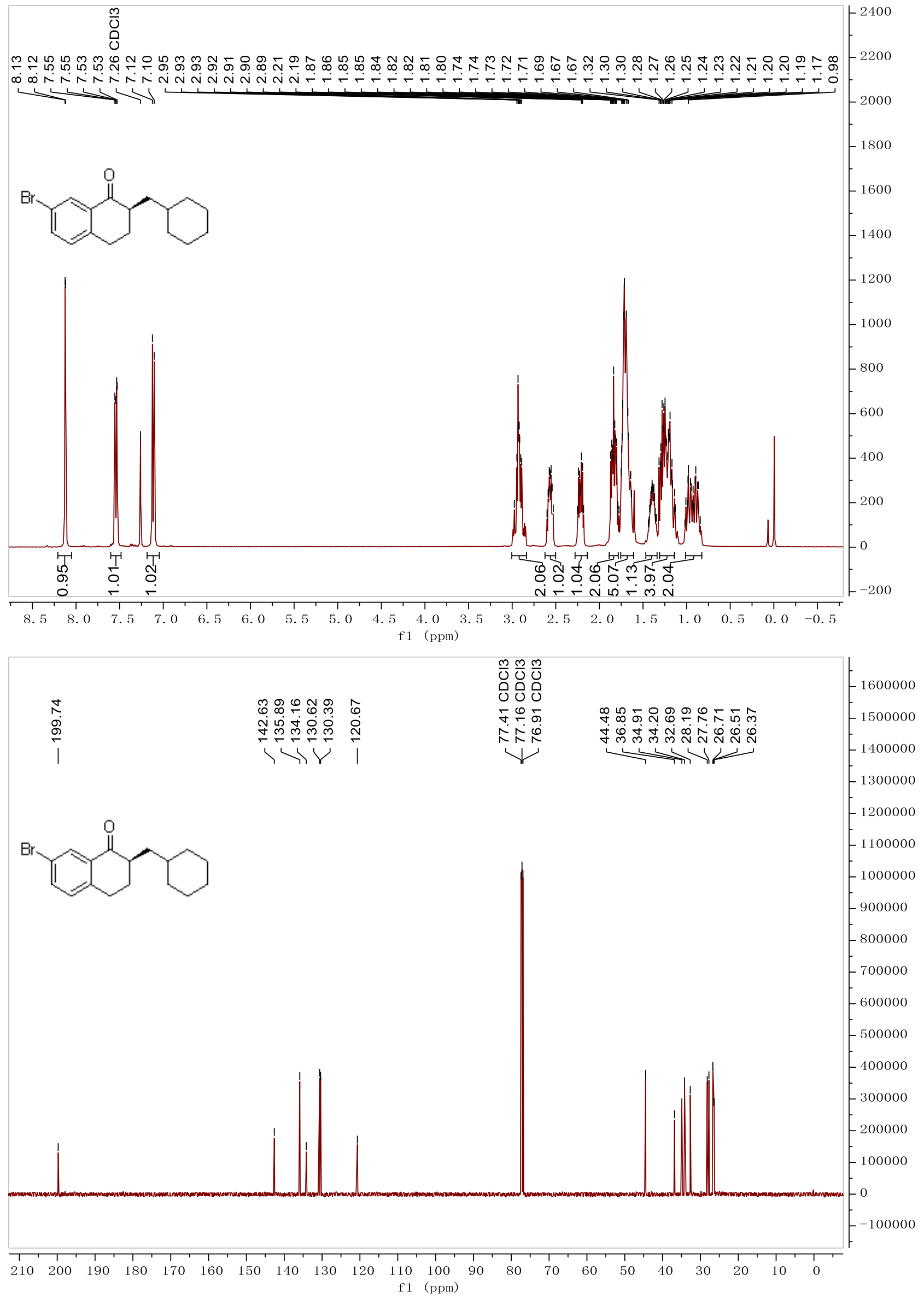




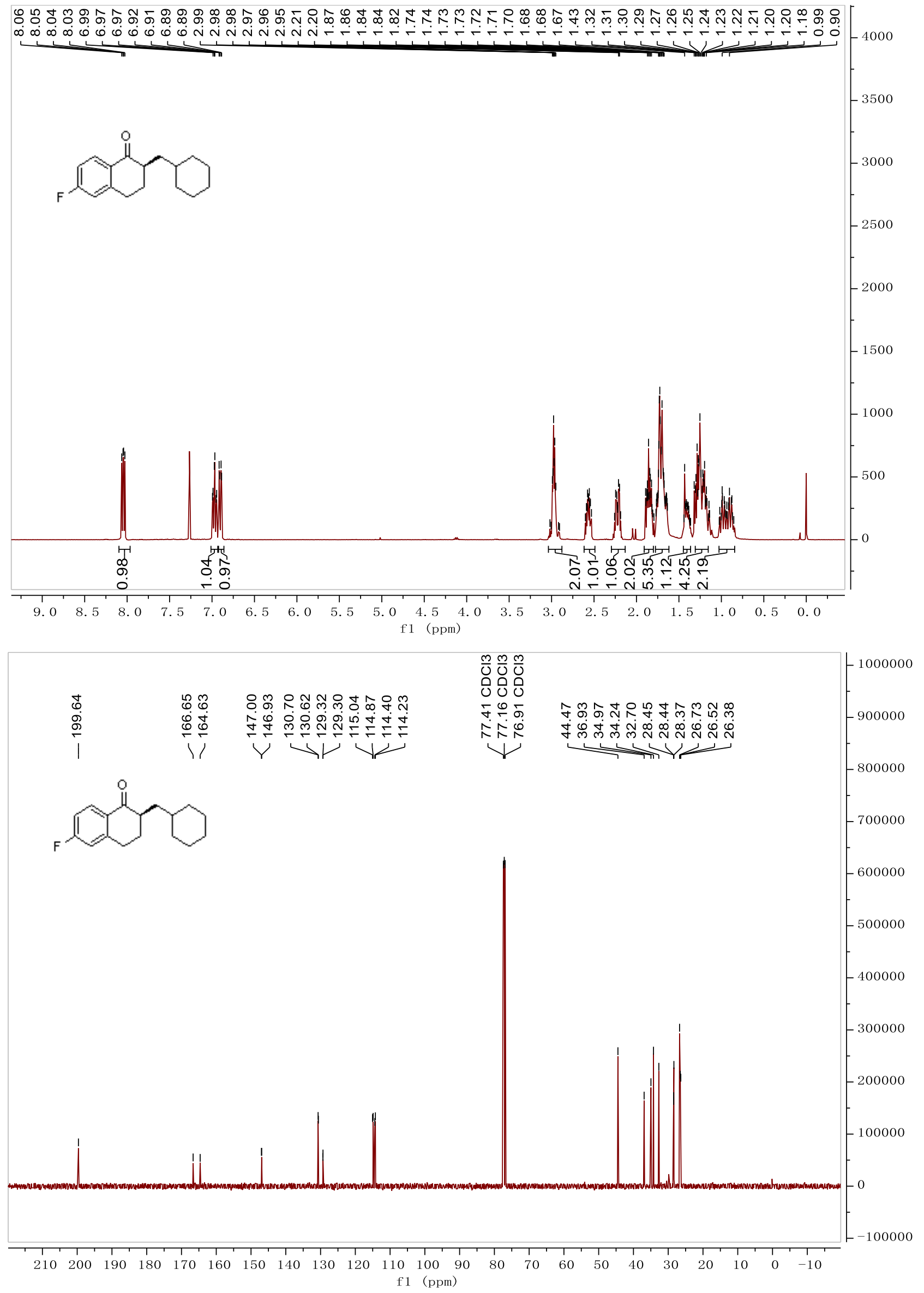



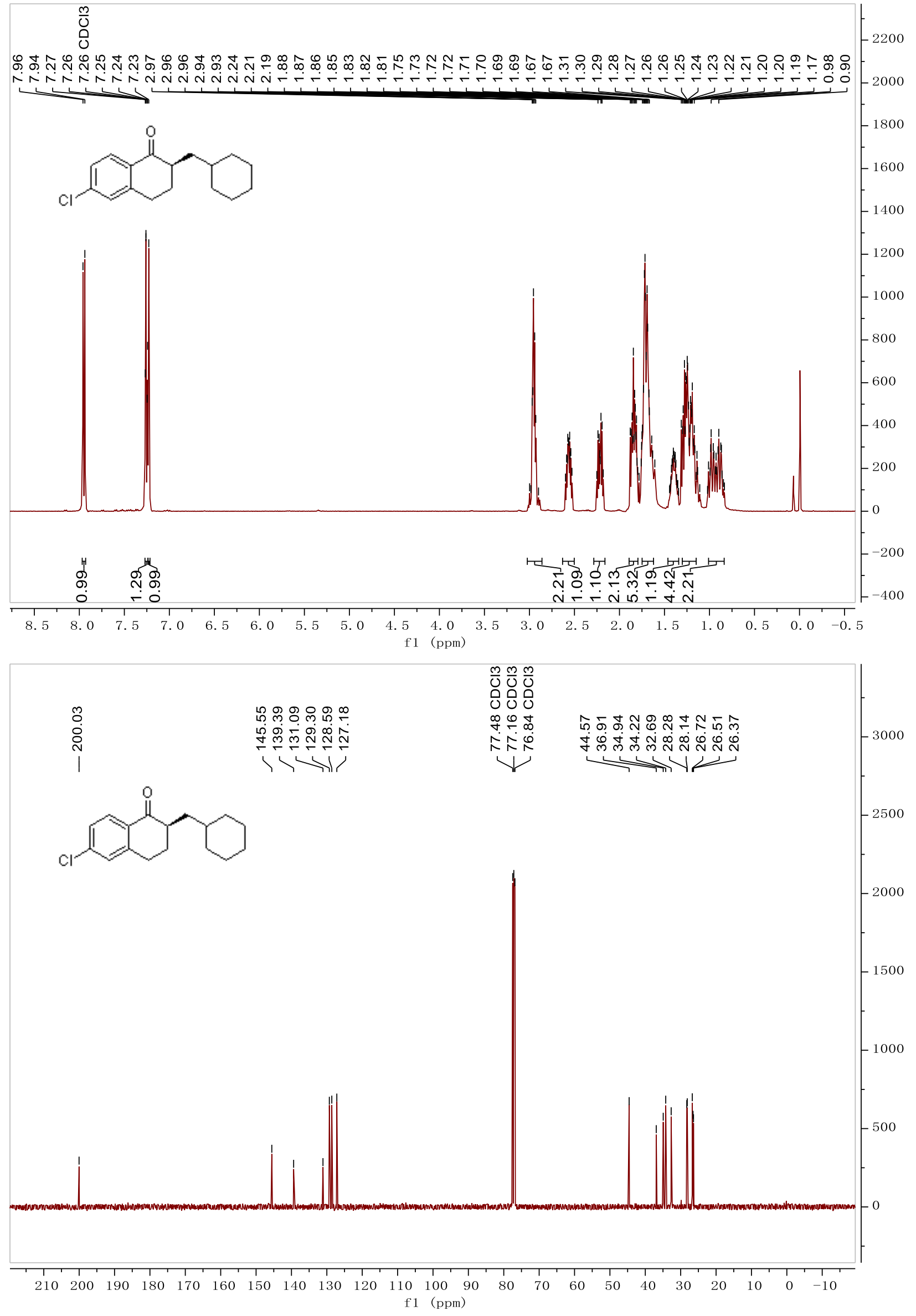


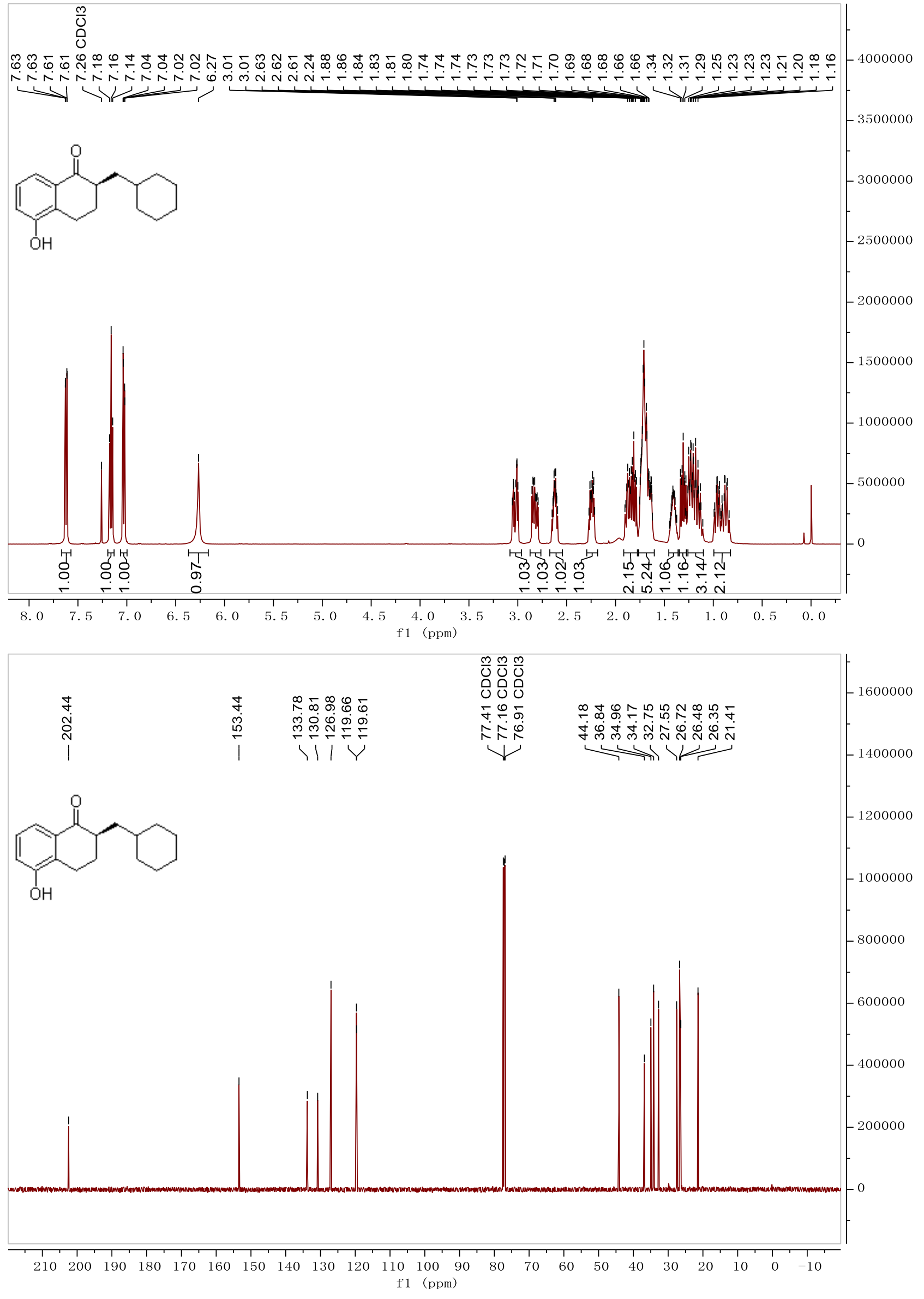




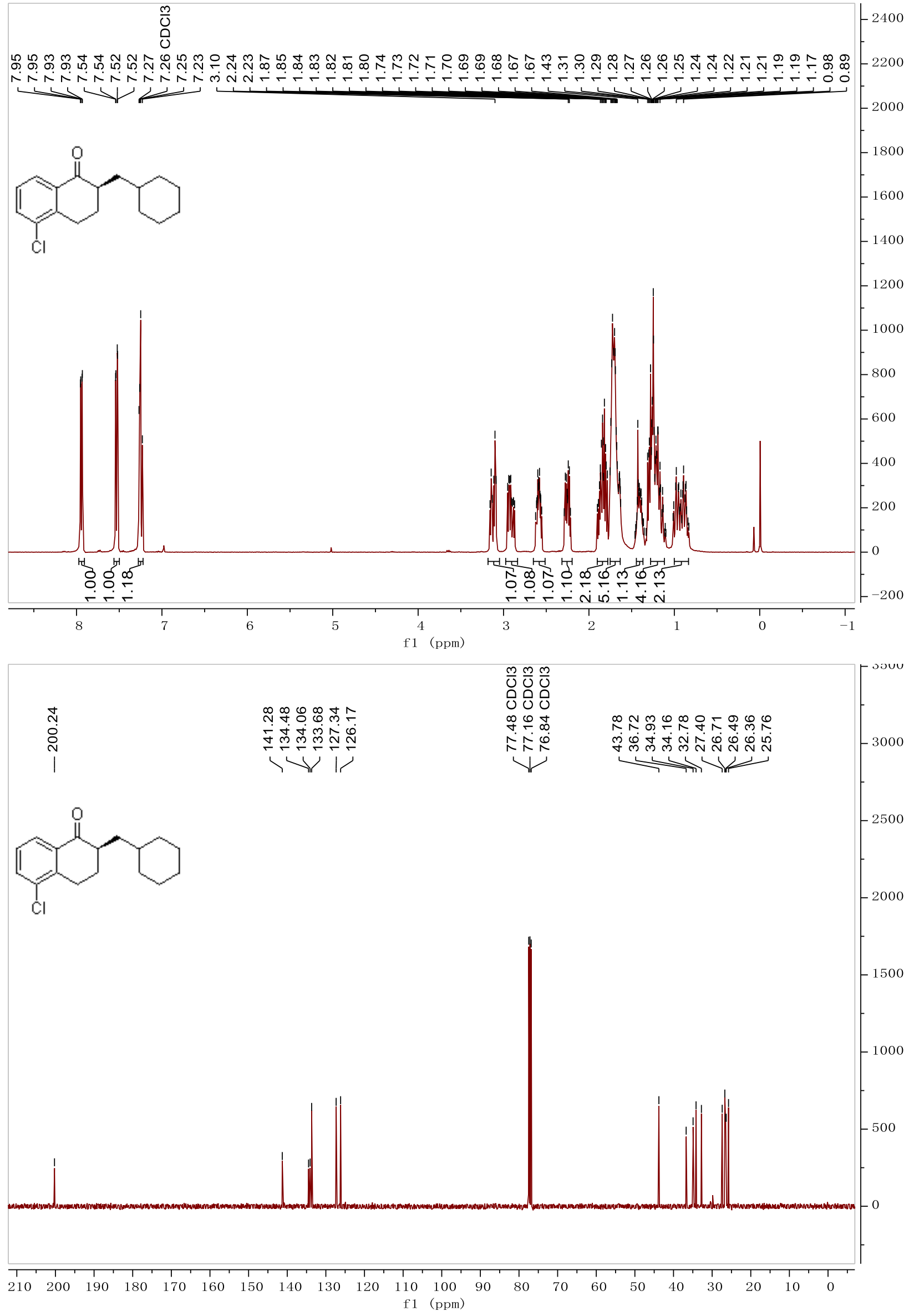



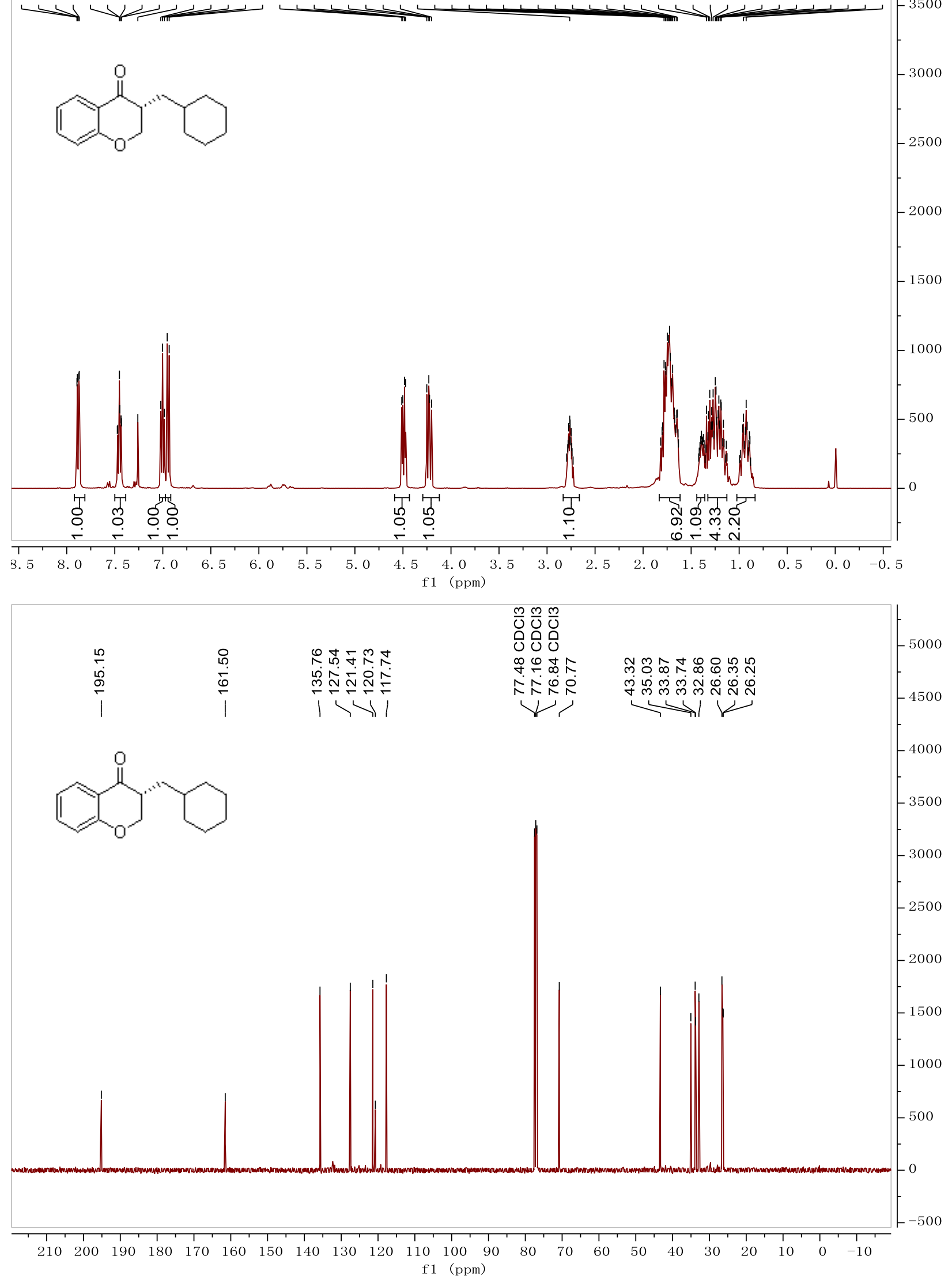


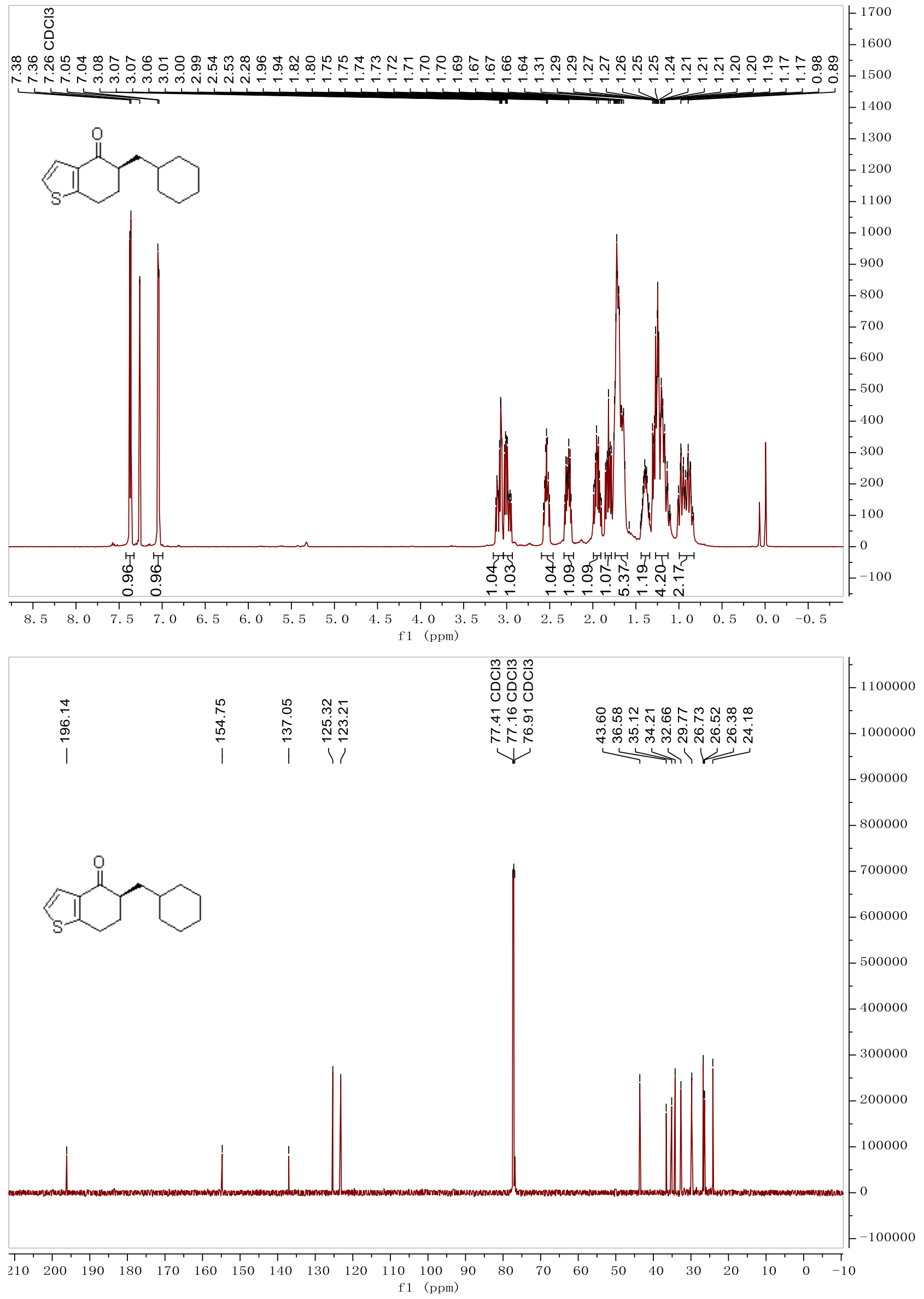




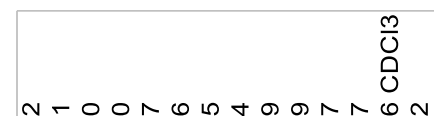

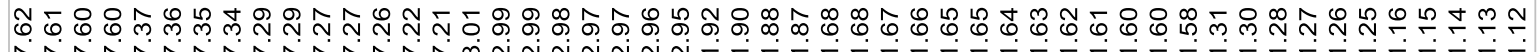

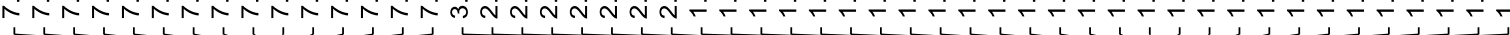<smiles>O=C1c2ccccc2CCCC1CC1CCCCC1</smiles>
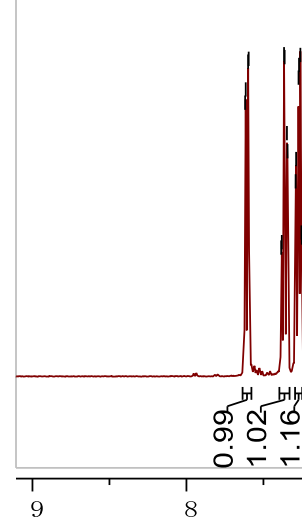

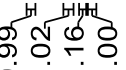
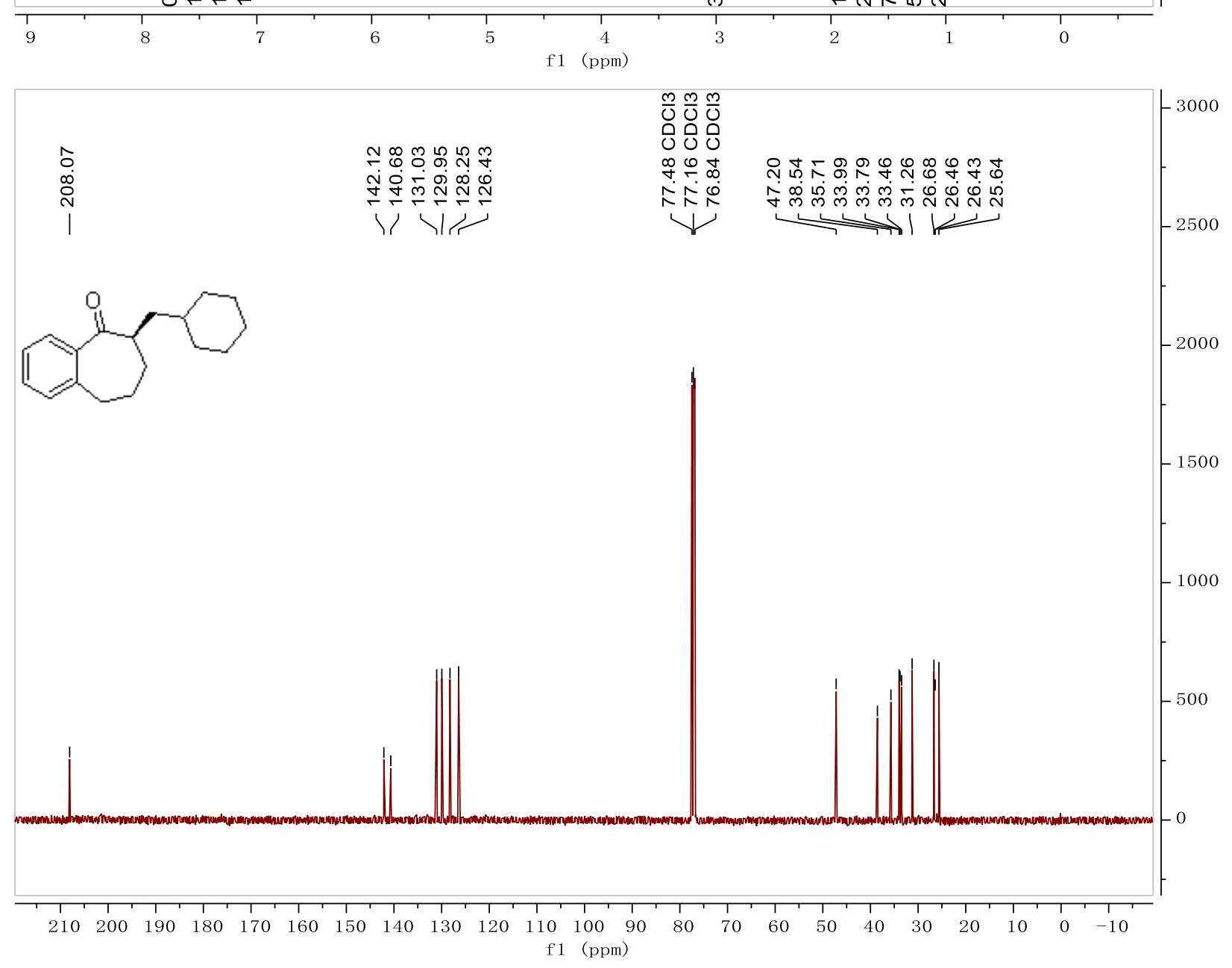


\section{O잉}

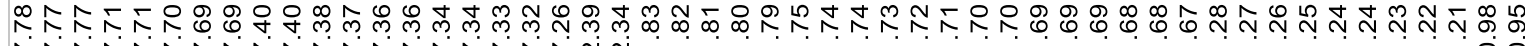

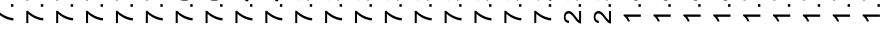

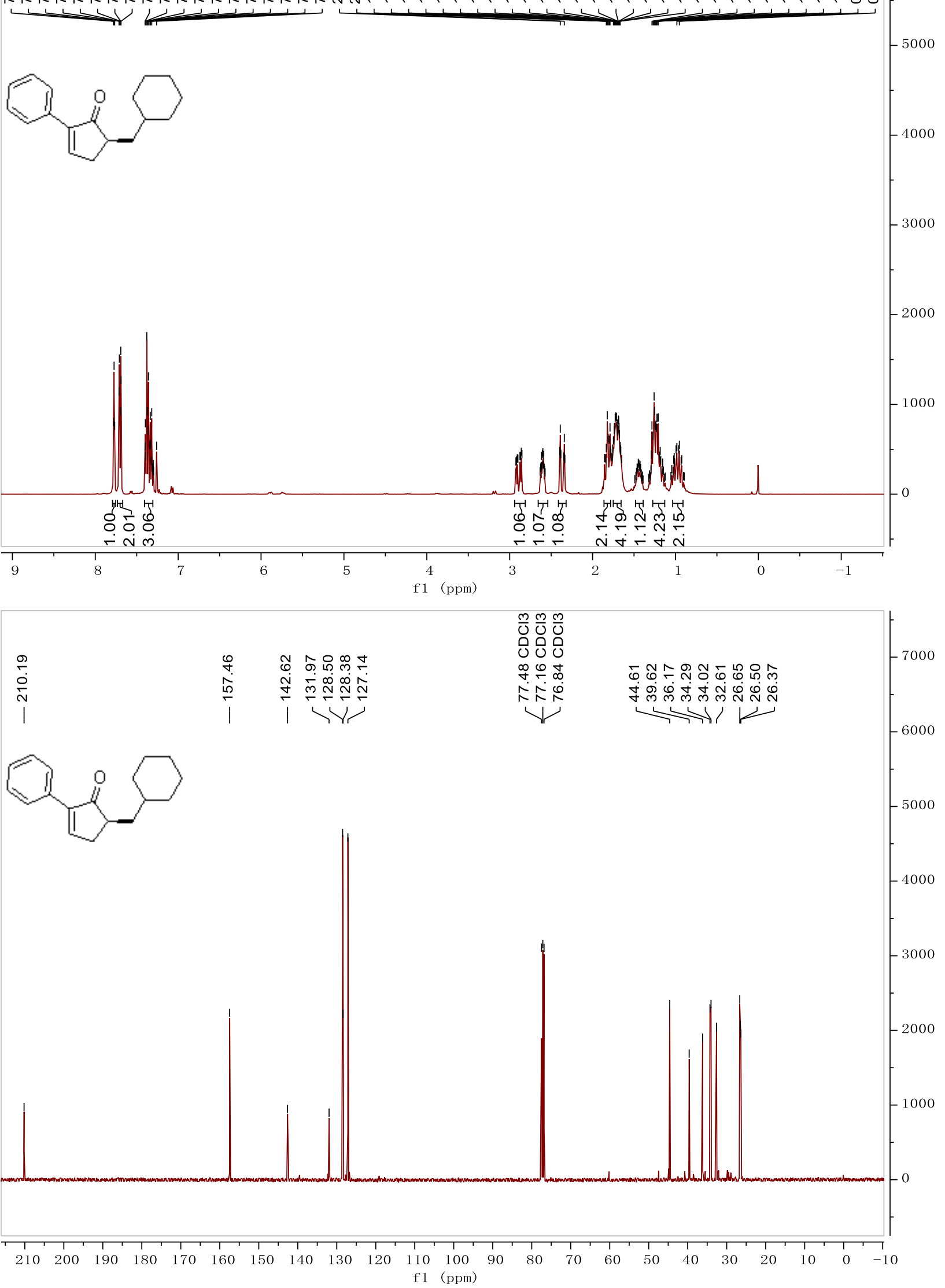




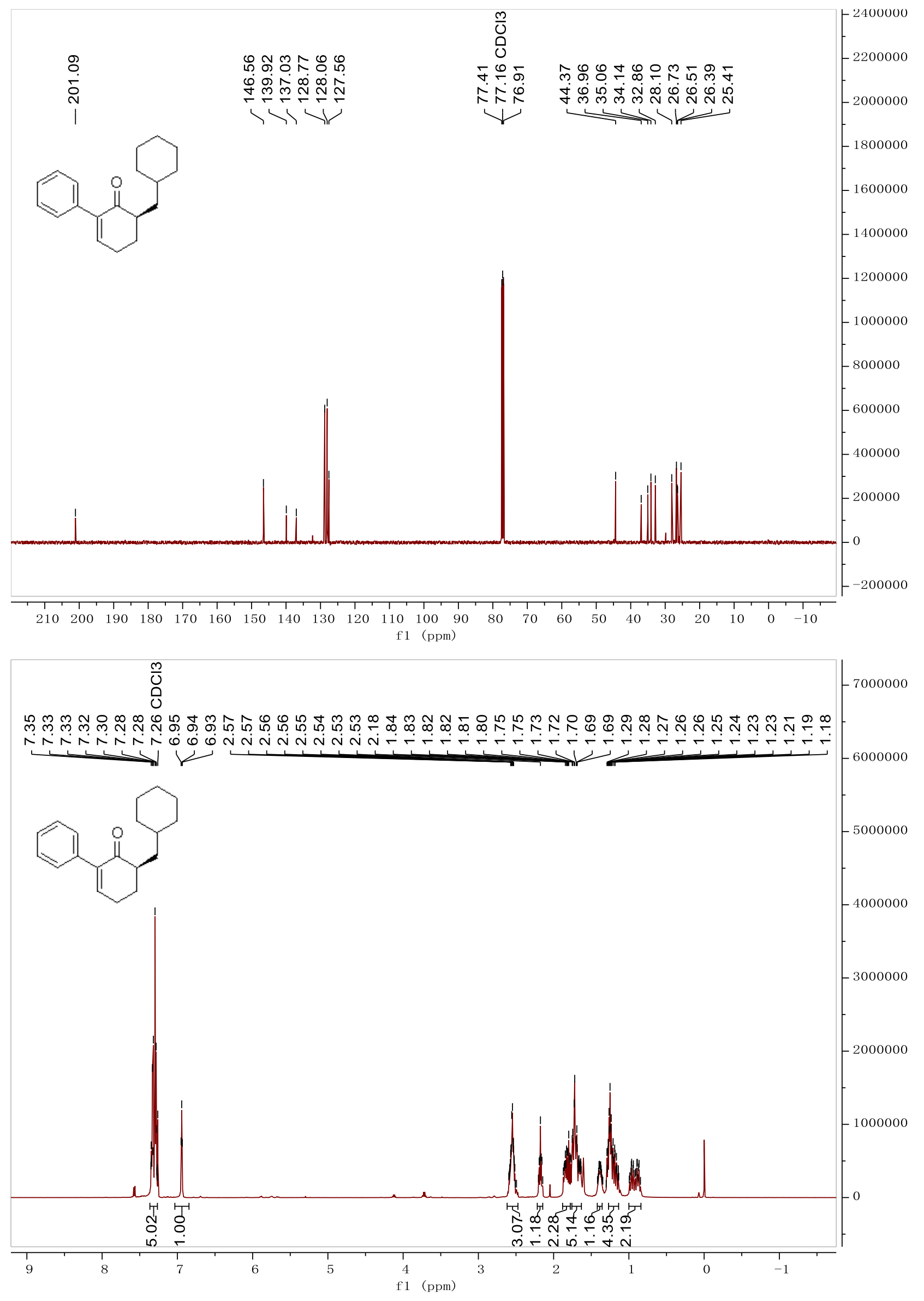



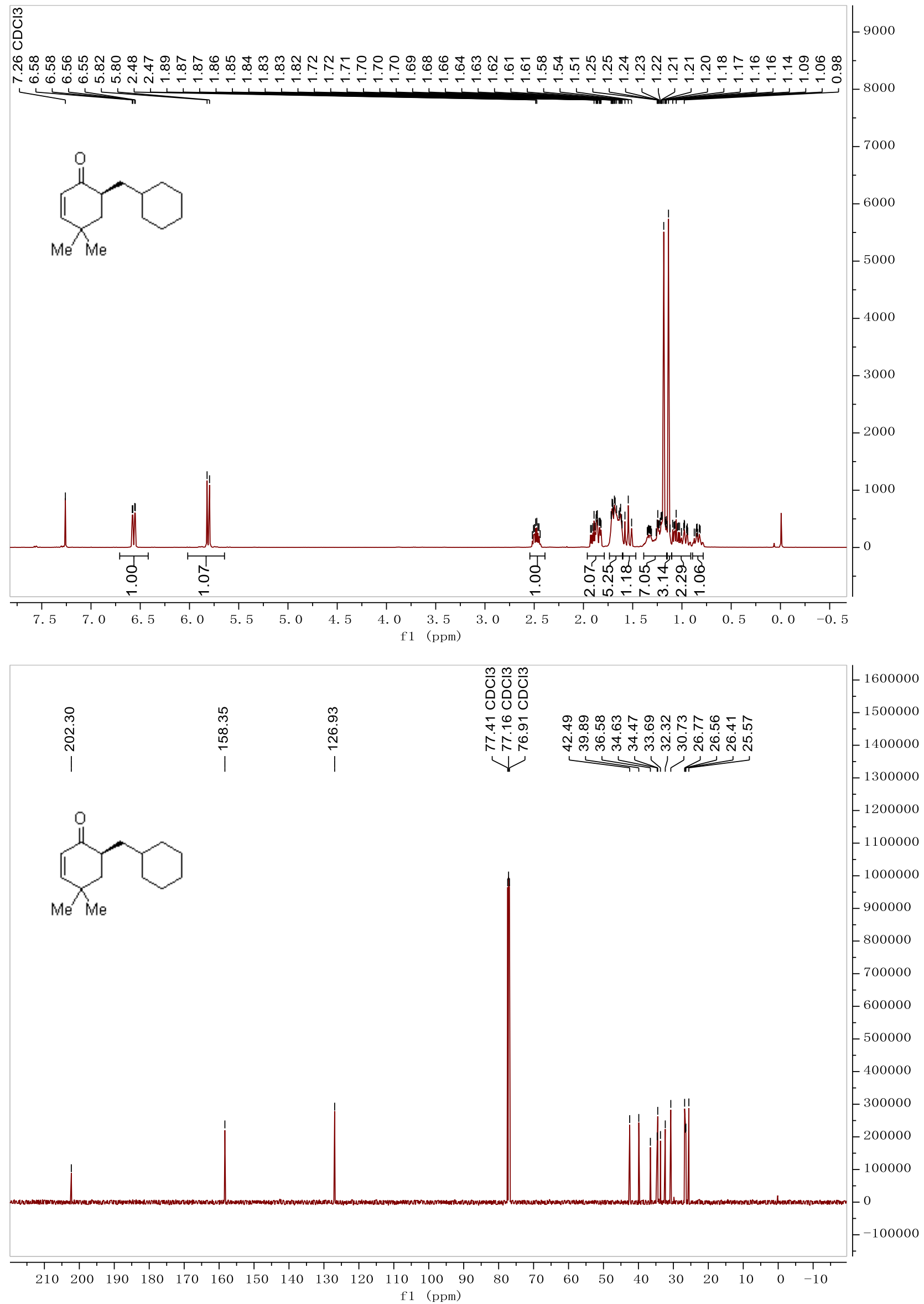
品

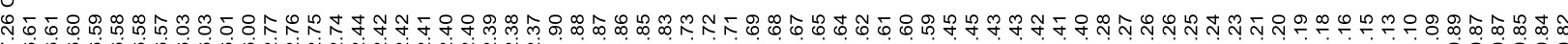

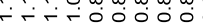
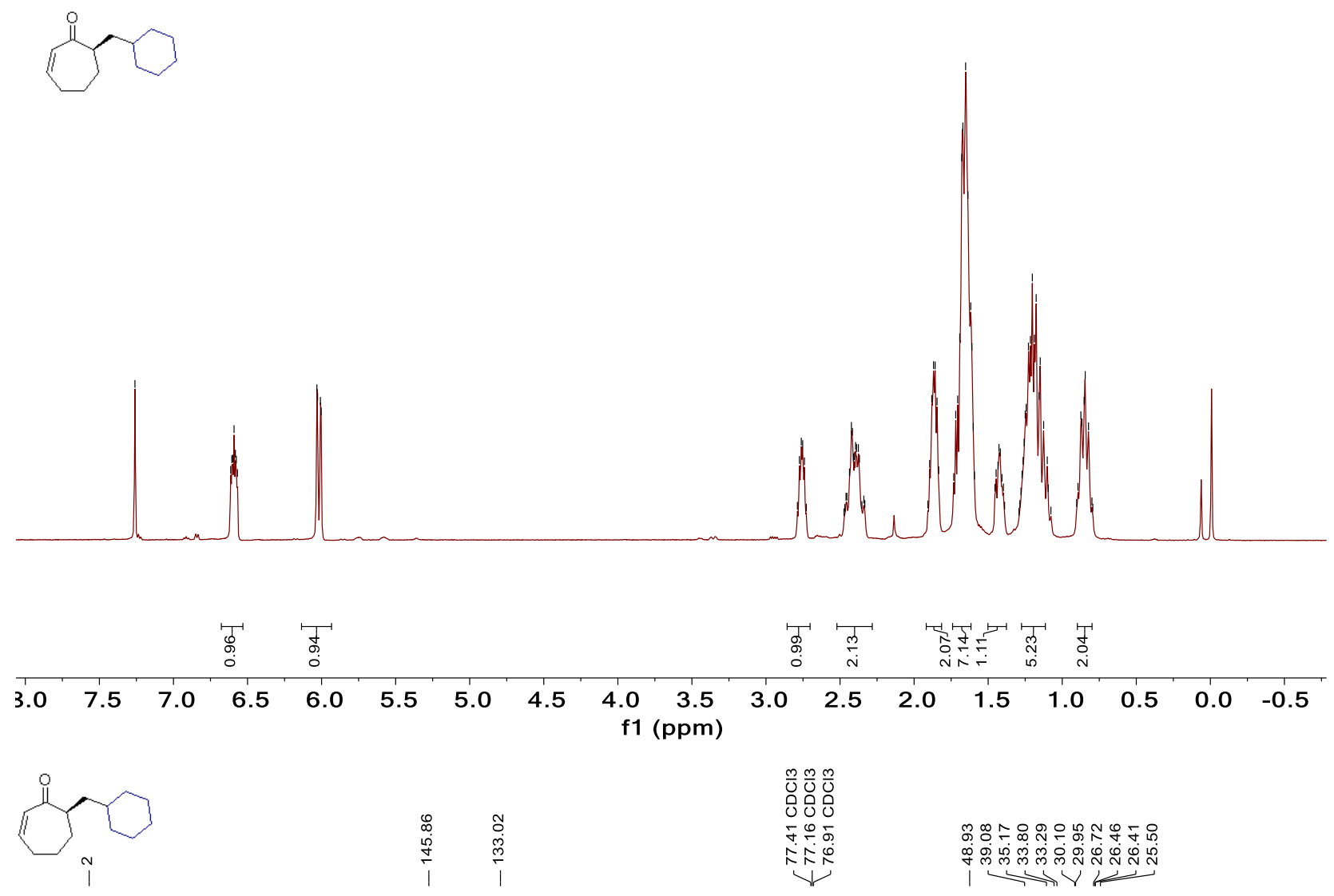

ถ⿻ำ

市

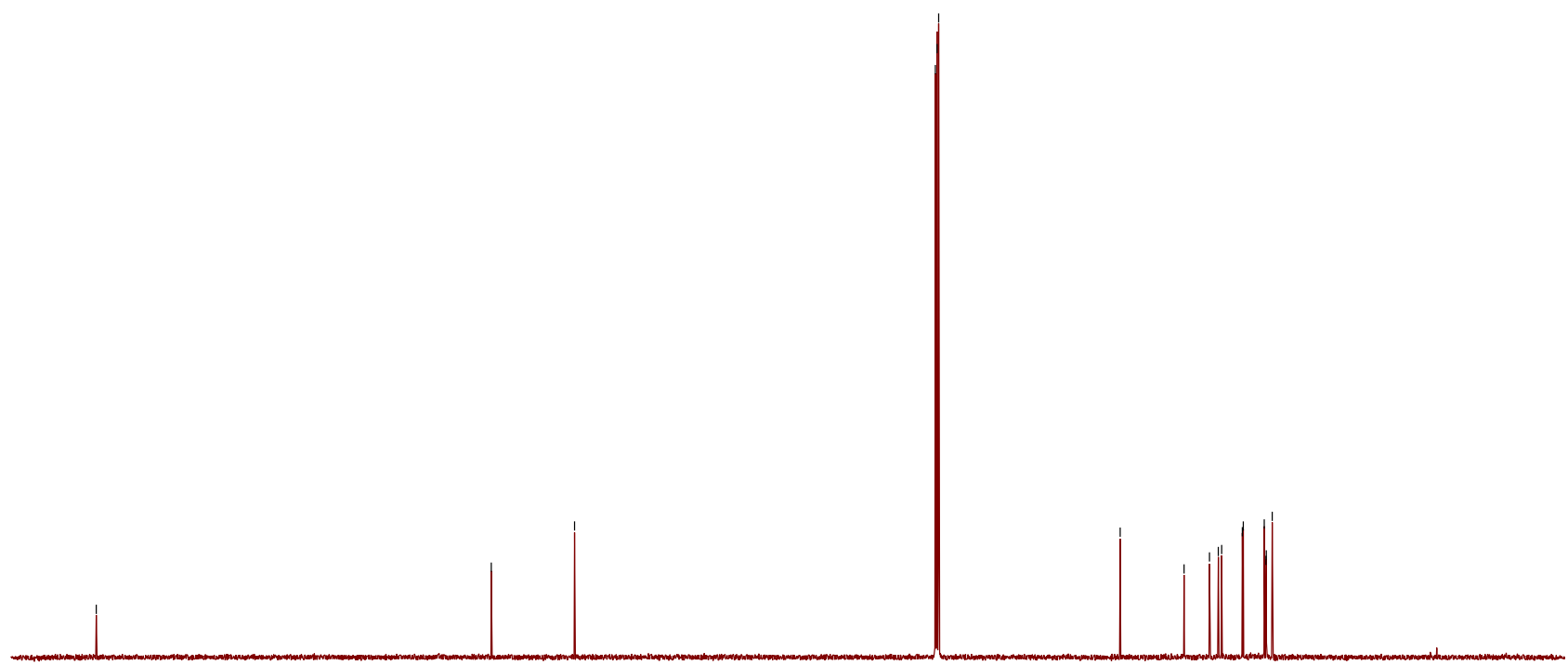

$210200190180170160150140130120110100 \quad 90 \quad 80 \quad 70 \quad 60 \begin{array}{lllllllll} & 40 & 30 & 20 & 10 & 0 & -10\end{array}$ f1 (ppm) 


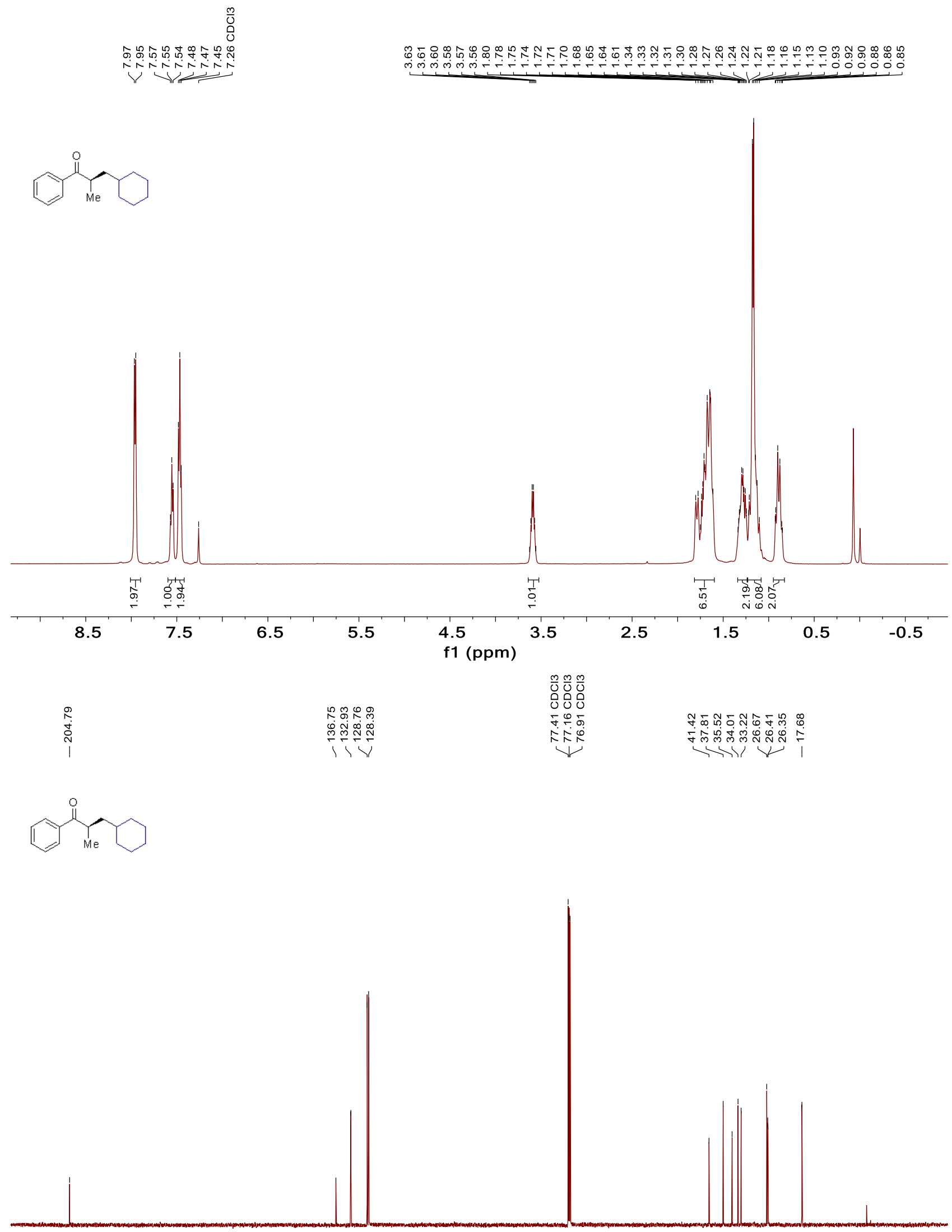

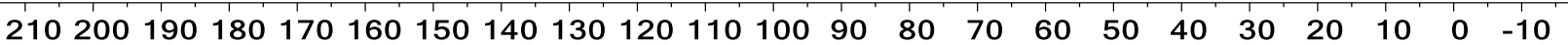
f1 (ppm) 

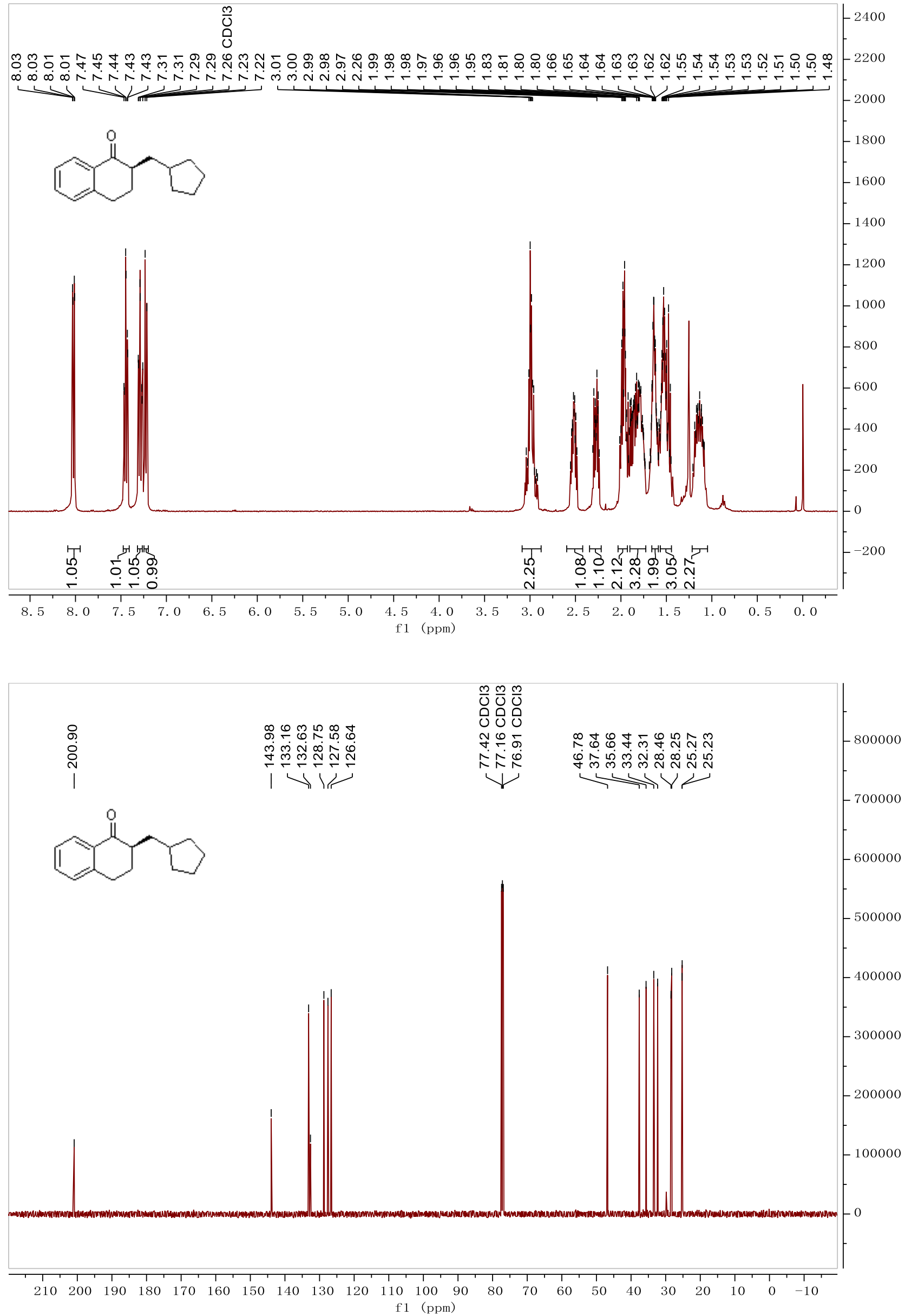


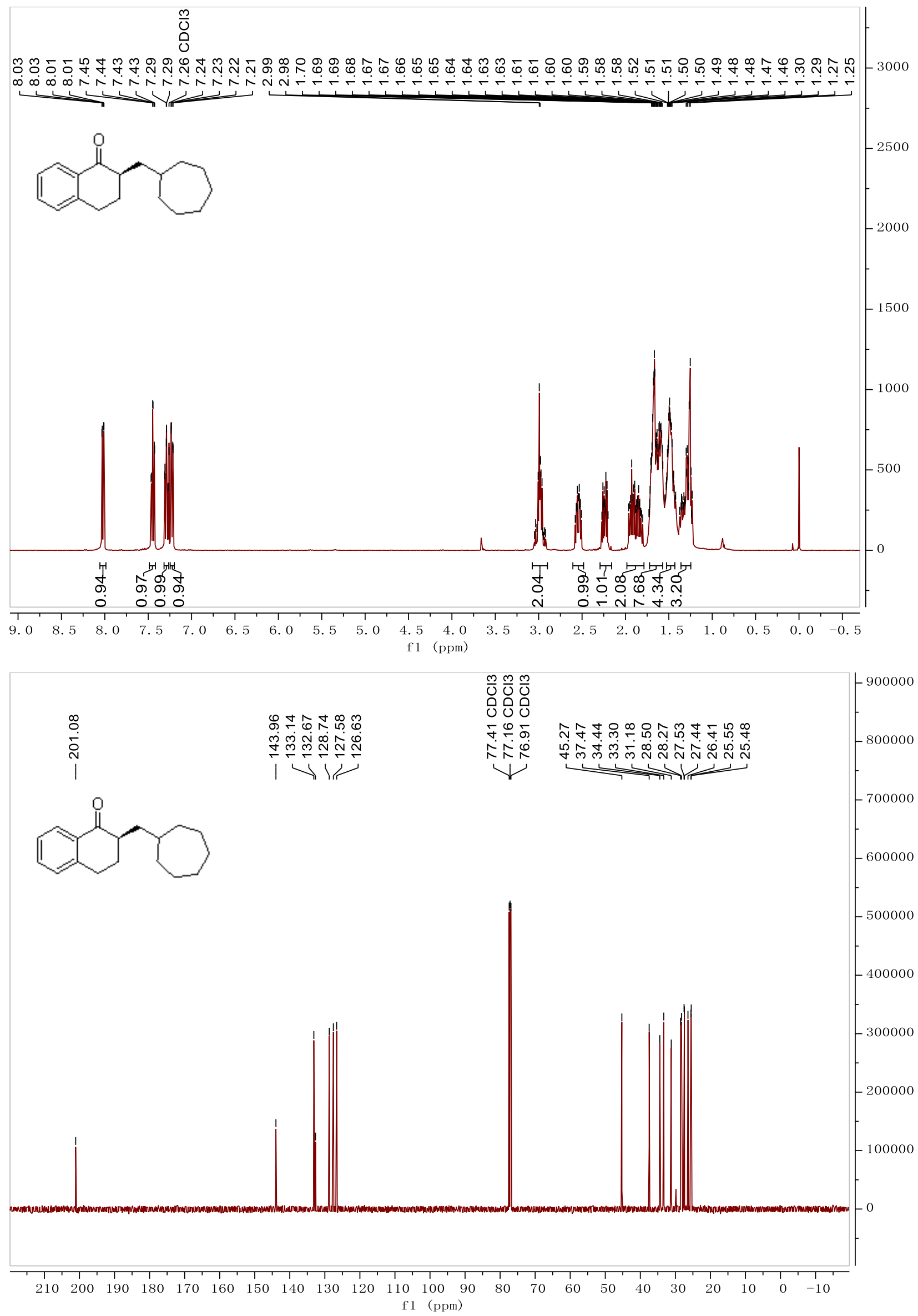



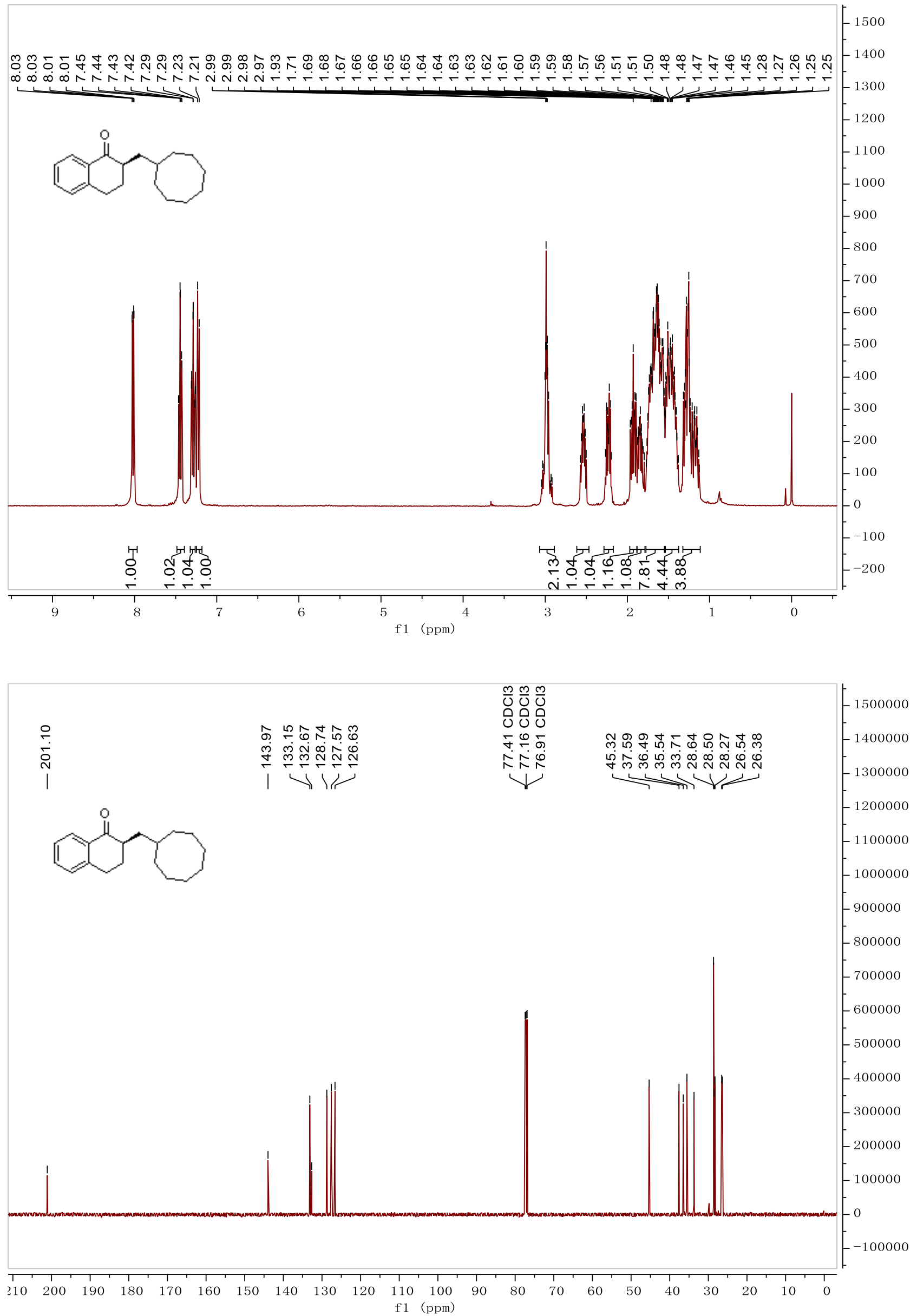


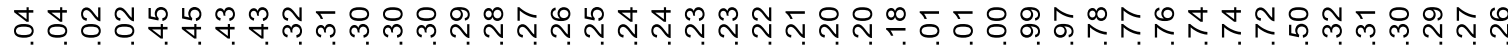
m

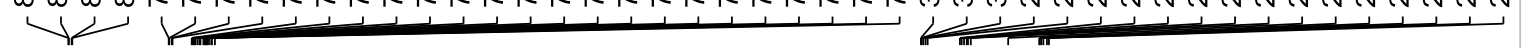<smiles>O=C1c2ccccc2CC[C@H]1CCc1ccccc1</smiles>
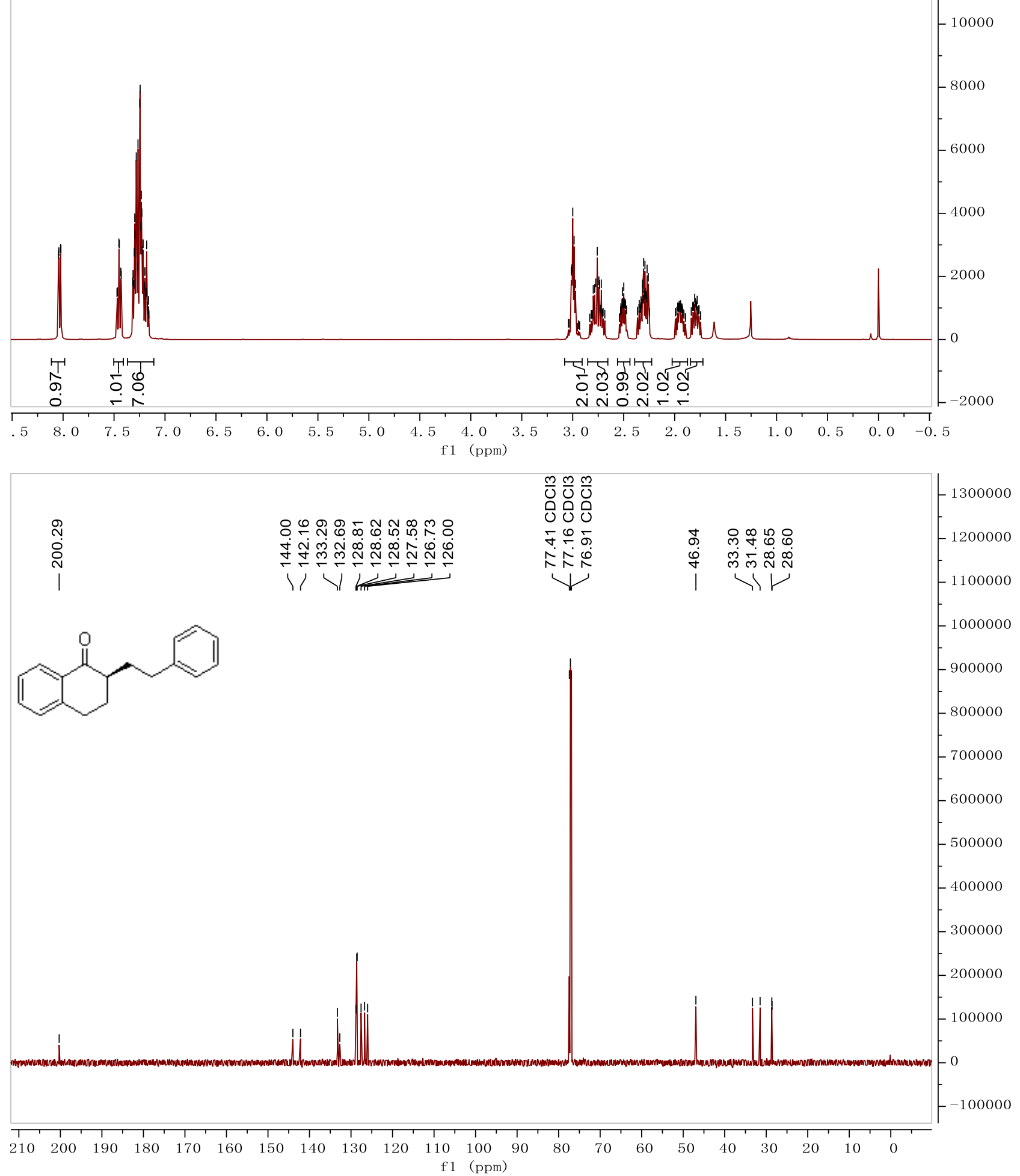


\section{嘀}

30000

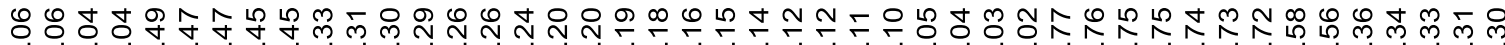
悉<smiles>CCCCCCC1CCc2ccccc2C1=O</smiles>
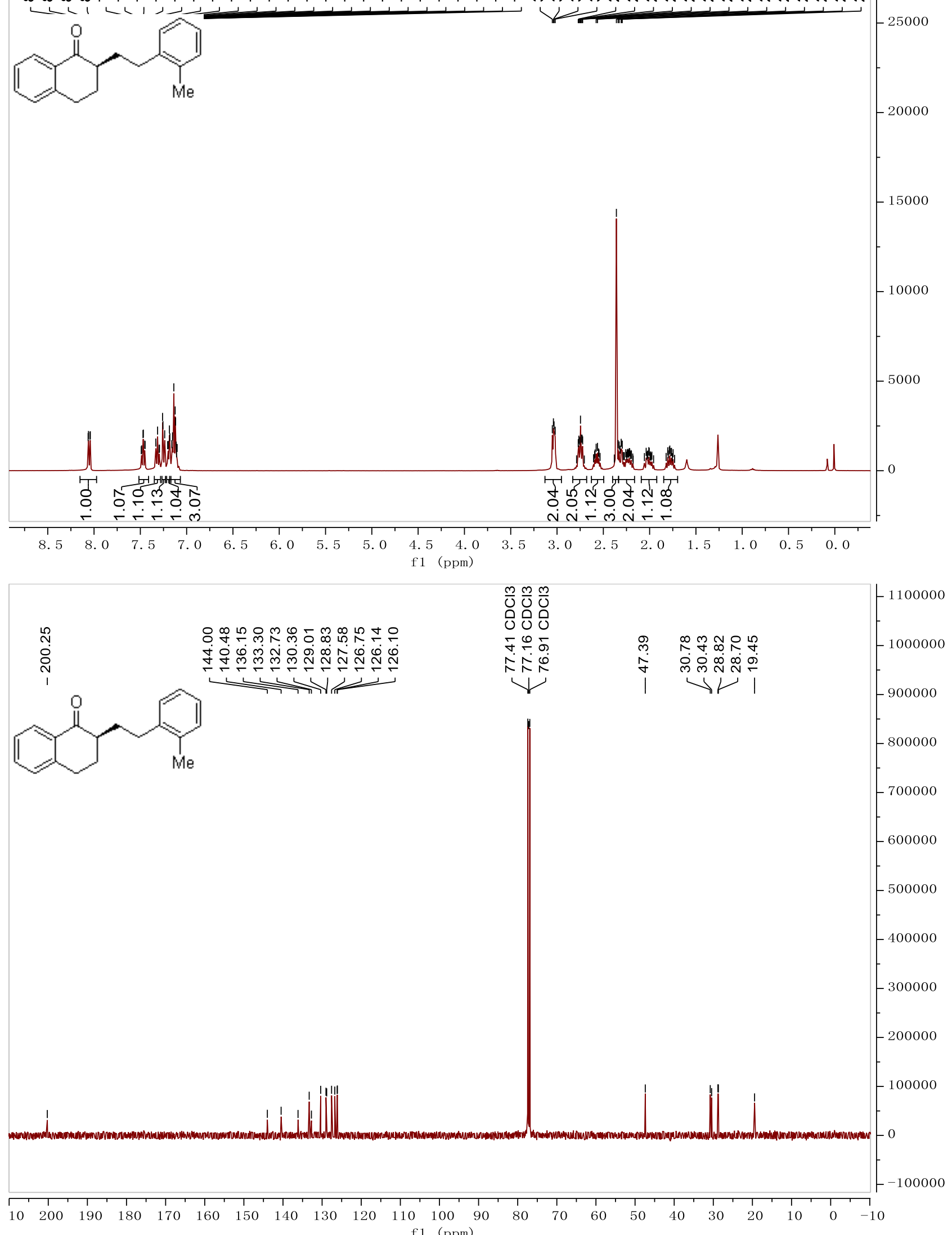


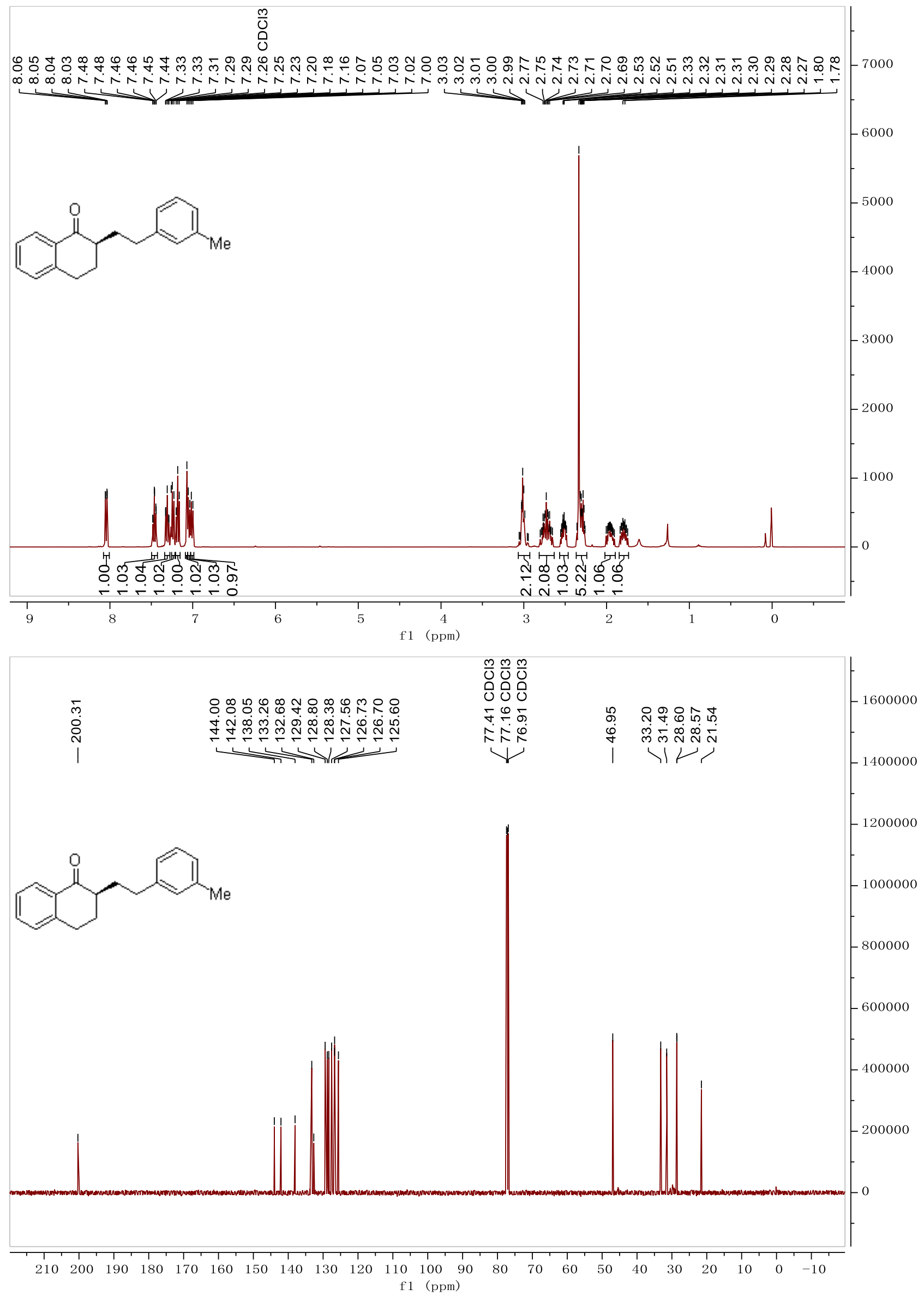




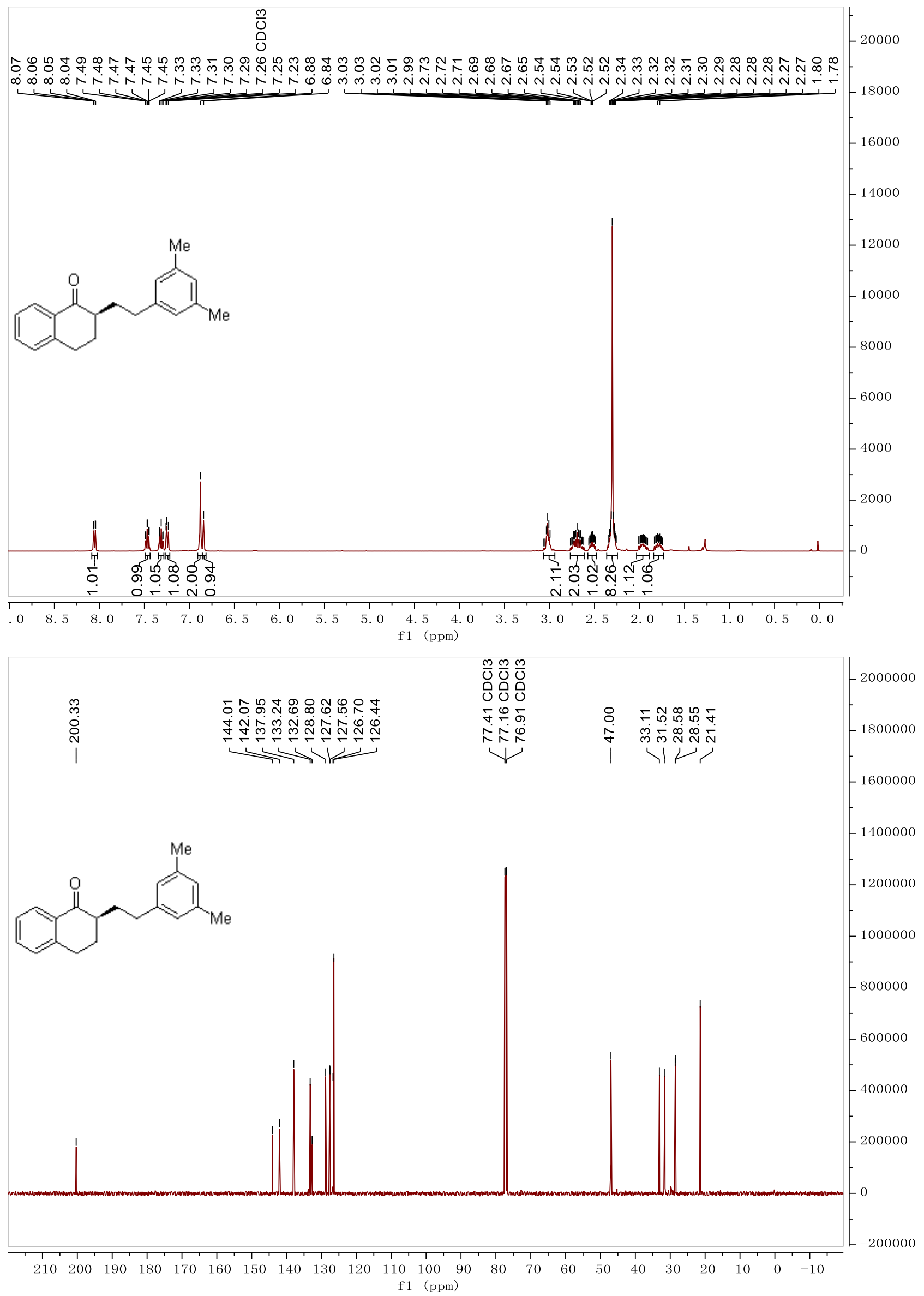




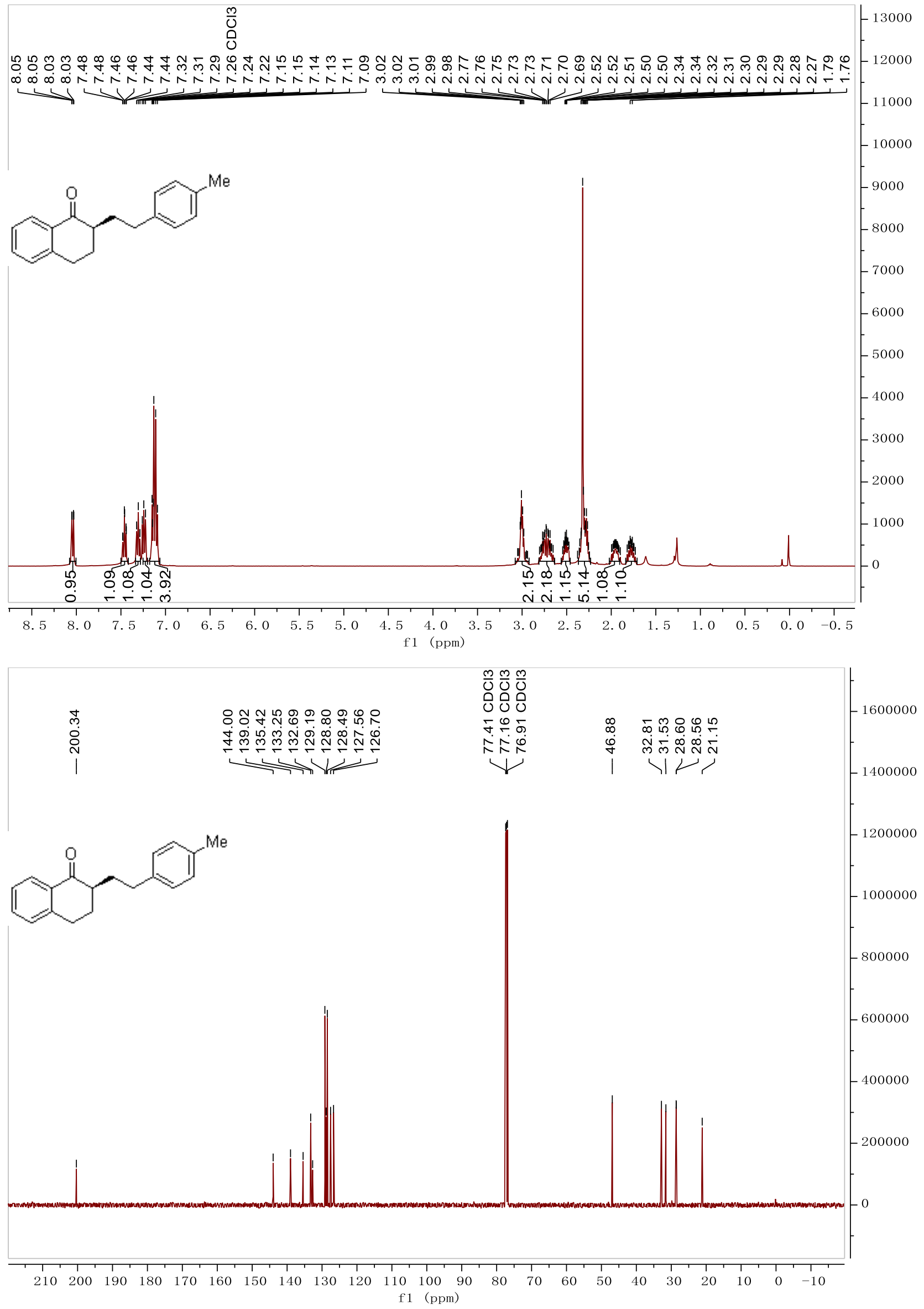



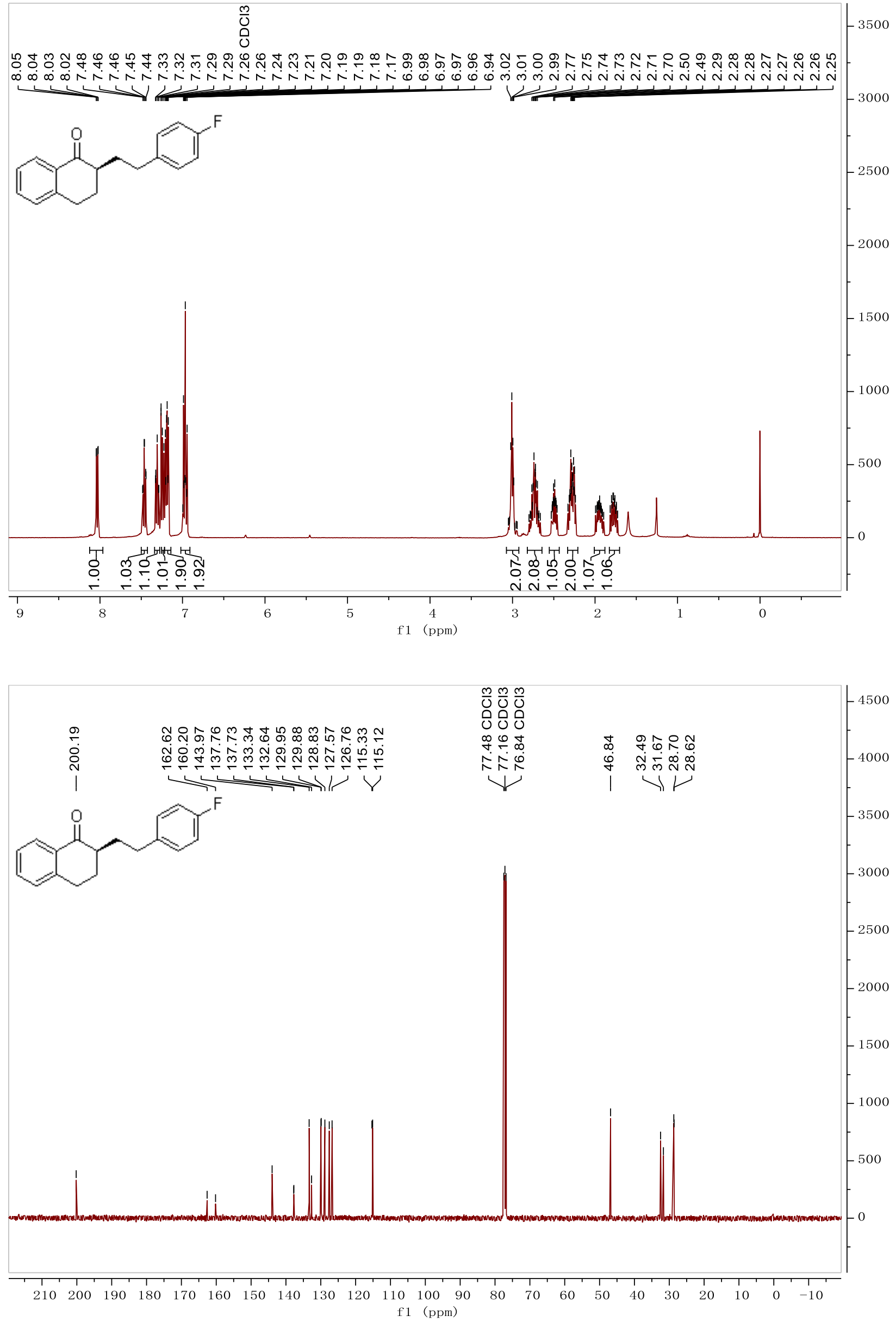


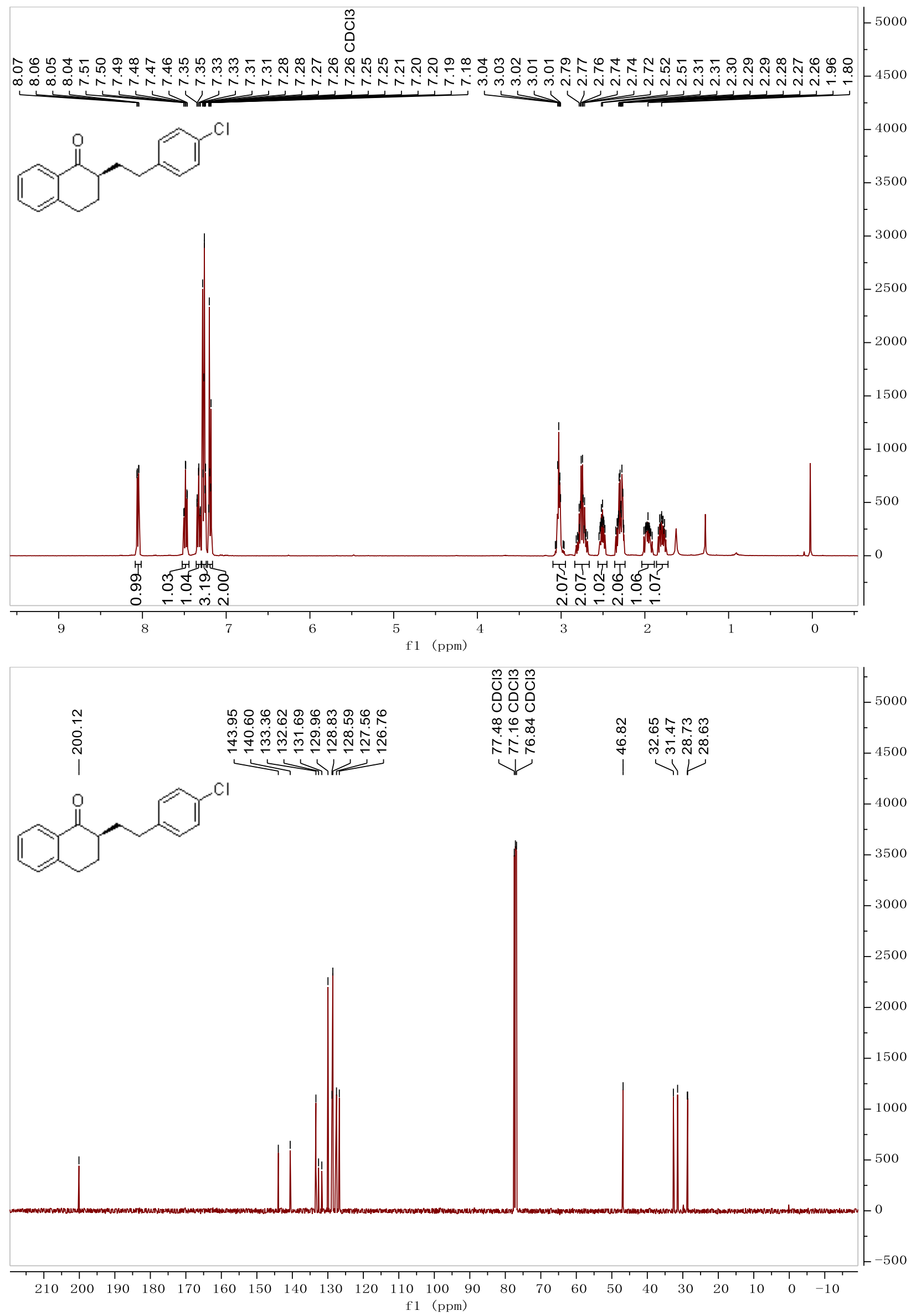



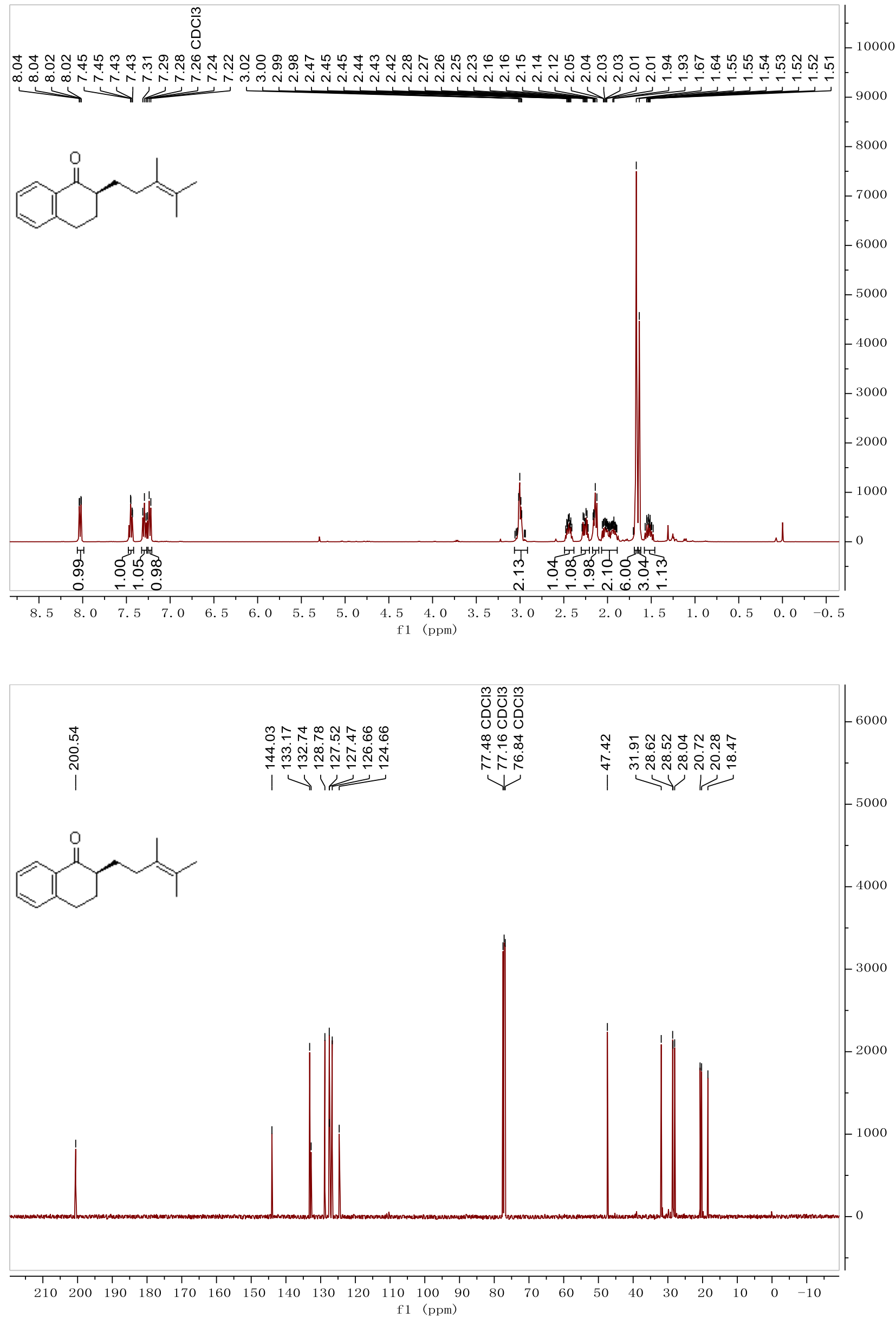


\section{$\frac{0}{0}$}

11000000

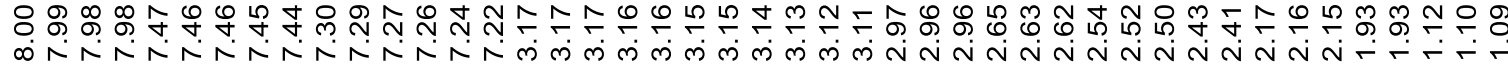
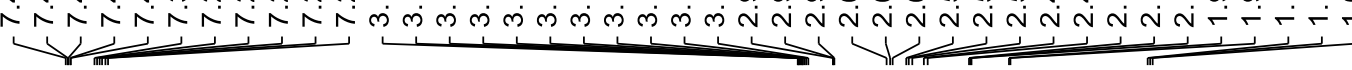<smiles>CCC(=O)C[C@H]1CCc2ccccc2C1=O</smiles>
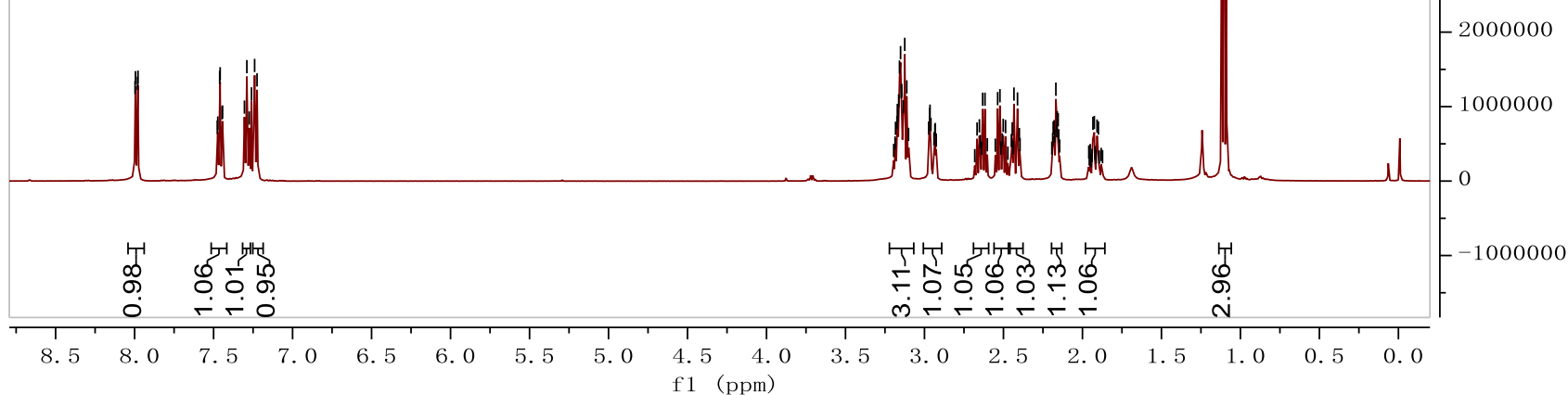

1 (ppm)

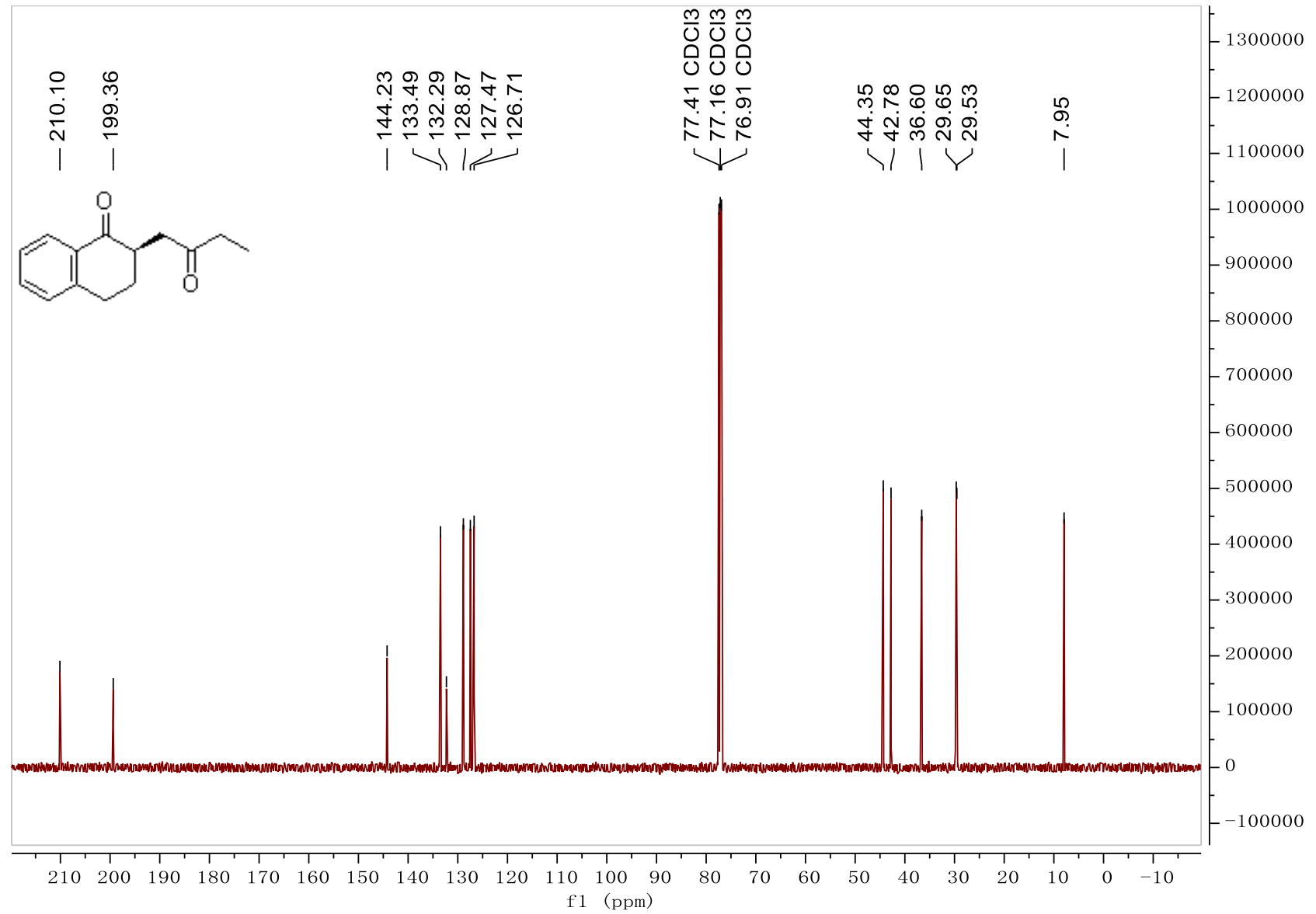




\section{HPLC spectra}
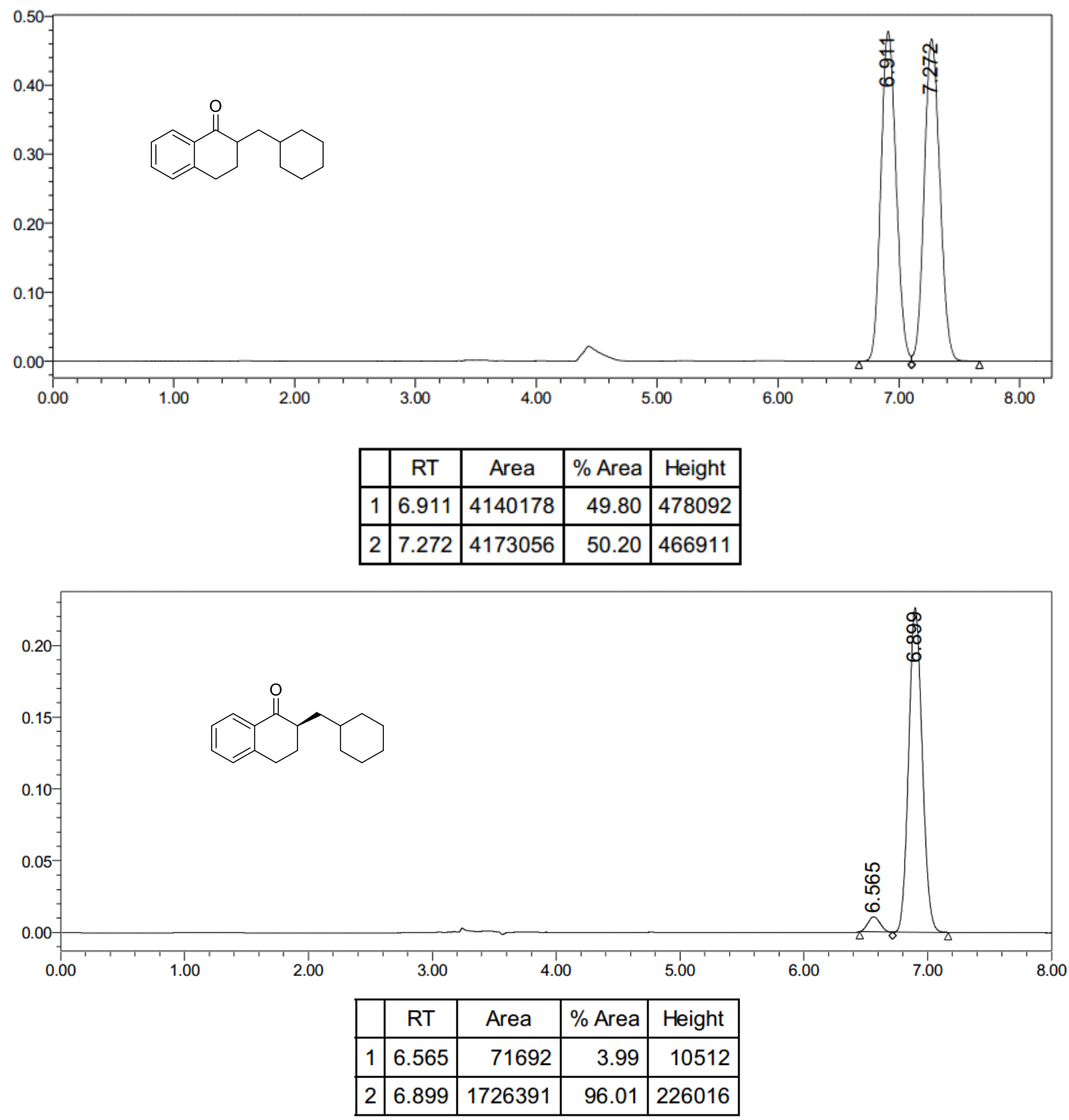

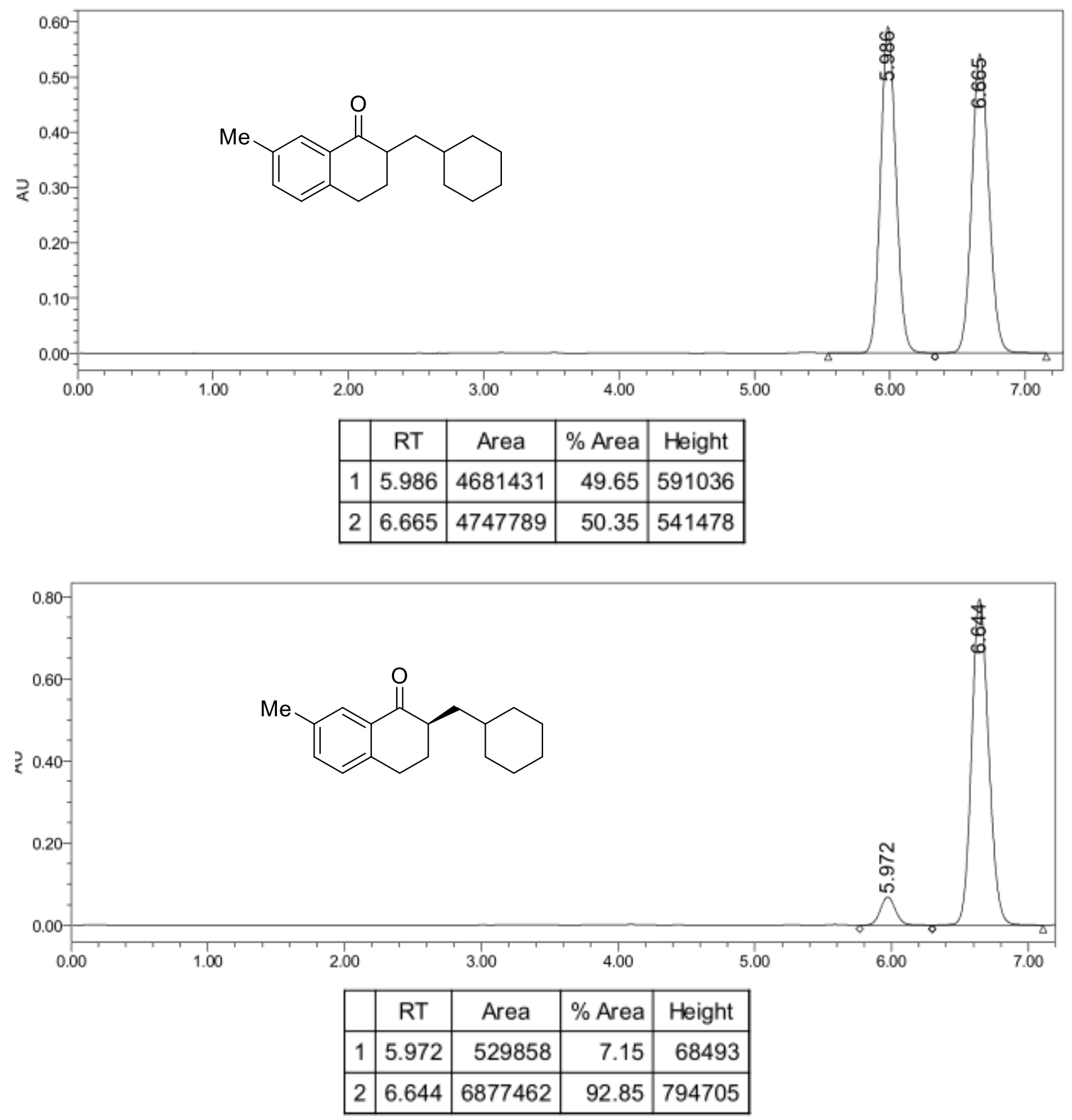


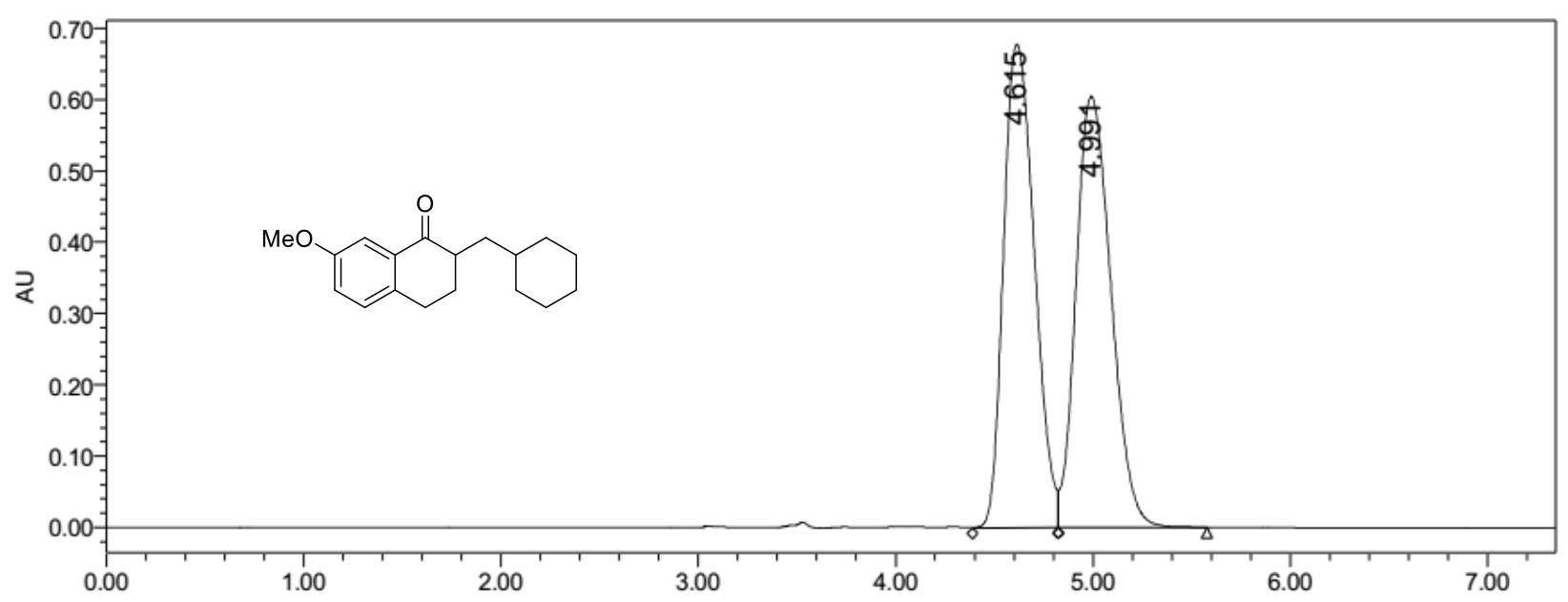

\begin{tabular}{|c|c|c|r|c|}
\hline & RT & Area & $\%$ Area & Height \\
\hline 1 & 4.615 & 7365027 & 49.59 & 677659 \\
\hline 2 & 4.991 & 7487813 & 50.41 & 604731 \\
\hline
\end{tabular}

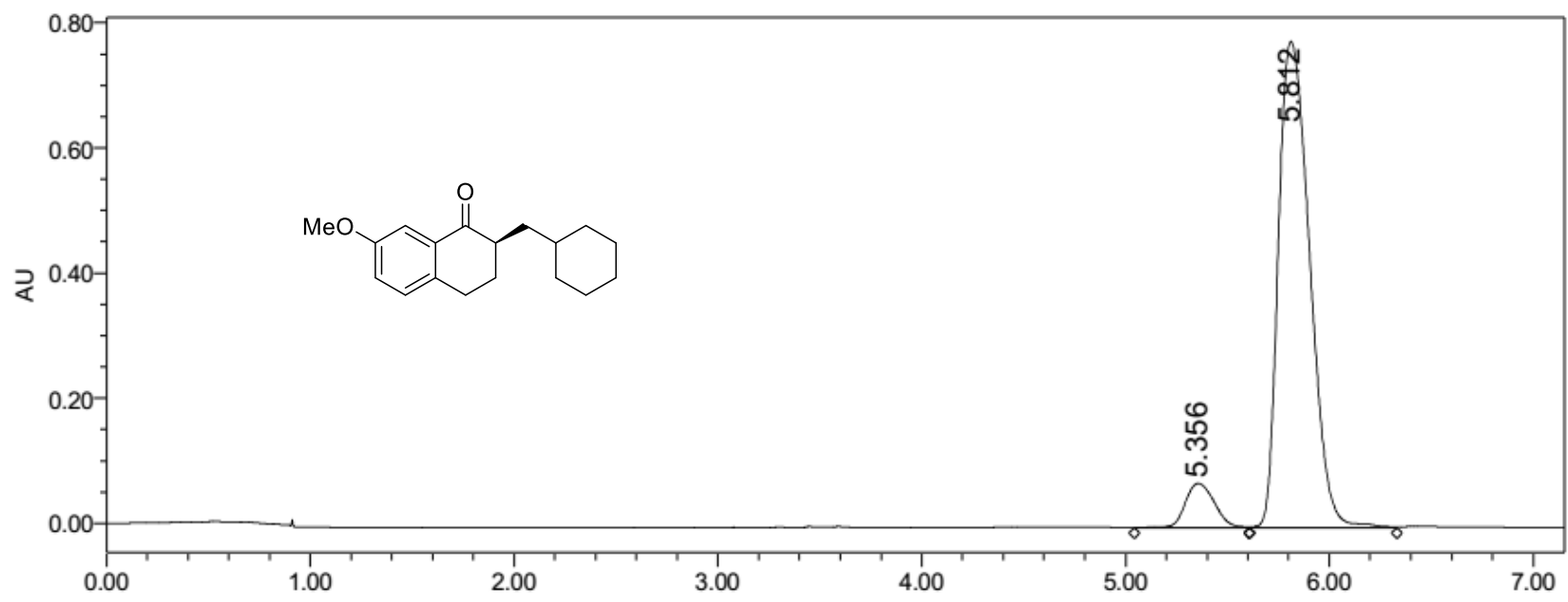

\begin{tabular}{|l|c|c|r|r|}
\hline & RT & Area & \% Area & Height \\
\hline 1 & 5.356 & 692162 & 7.73 & 70253 \\
\hline 2 & 5.812 & 8263888 & 92.27 & 776735 \\
\hline
\end{tabular}




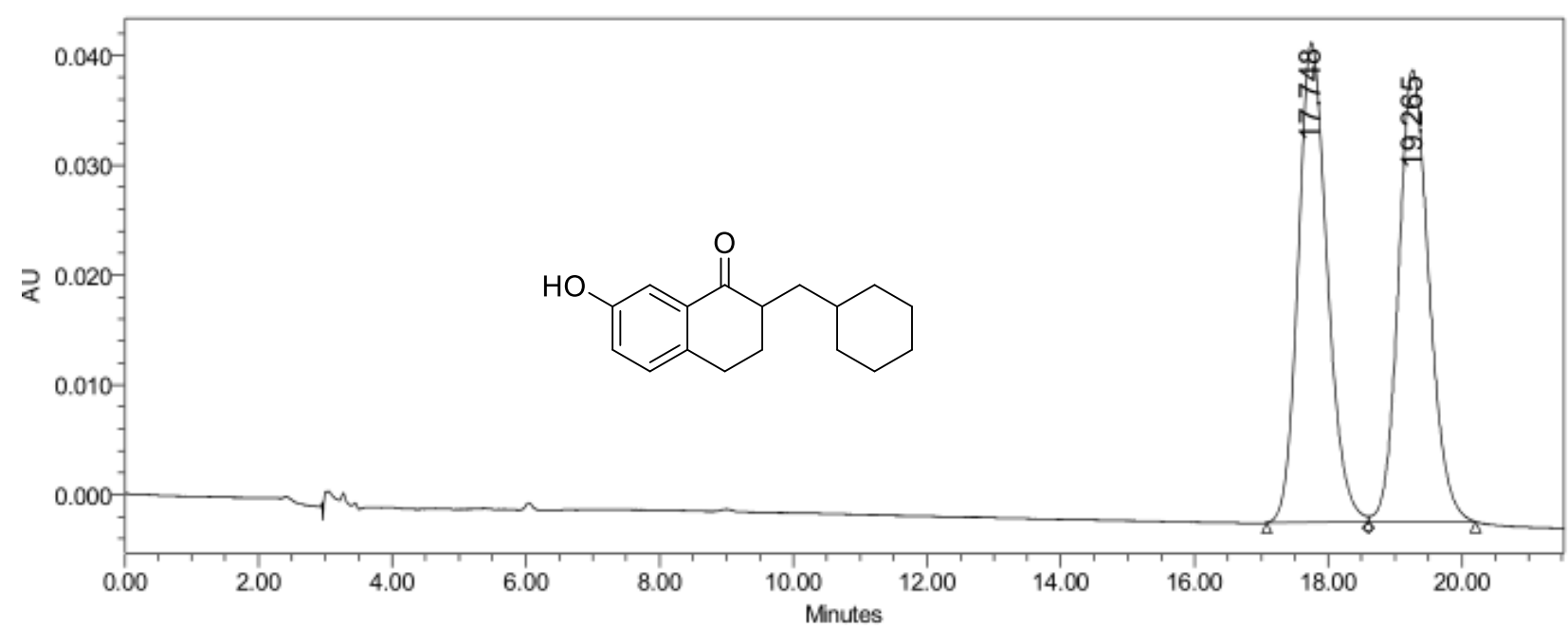

\begin{tabular}{|c|c|c|r|c|}
\hline & RT & Area & $\%$ Area & Height \\
\hline 1 & 17.748 & 1320390 & 50.28 & 43686 \\
\hline 2 & 19.265 & 1305755 & 49.72 & 41136 \\
\hline
\end{tabular}

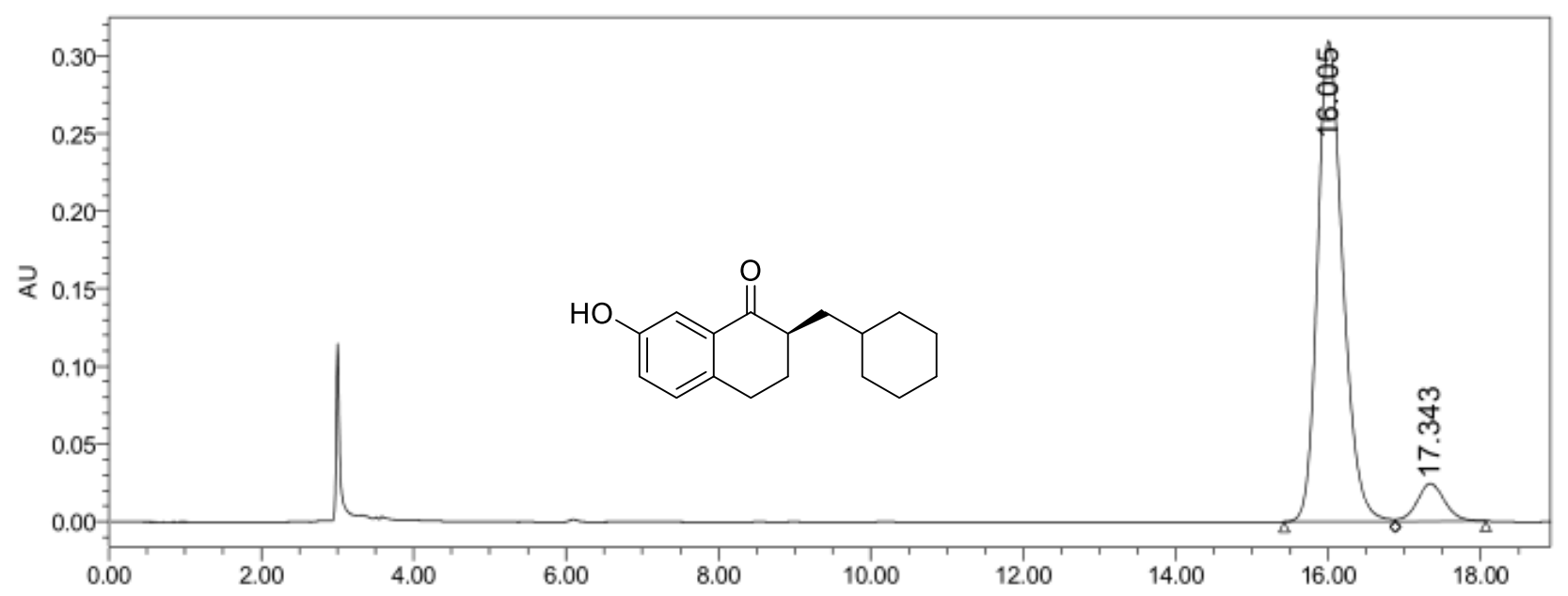

\begin{tabular}{|l|c|c|r|r|}
\hline & RT & Area & $\%$ Area & Height \\
\hline 1 & 16.005 & 7197959 & 92.15 & 309703 \\
\hline 2 & 17.343 & 613233 & 7.85 & 24284 \\
\hline
\end{tabular}




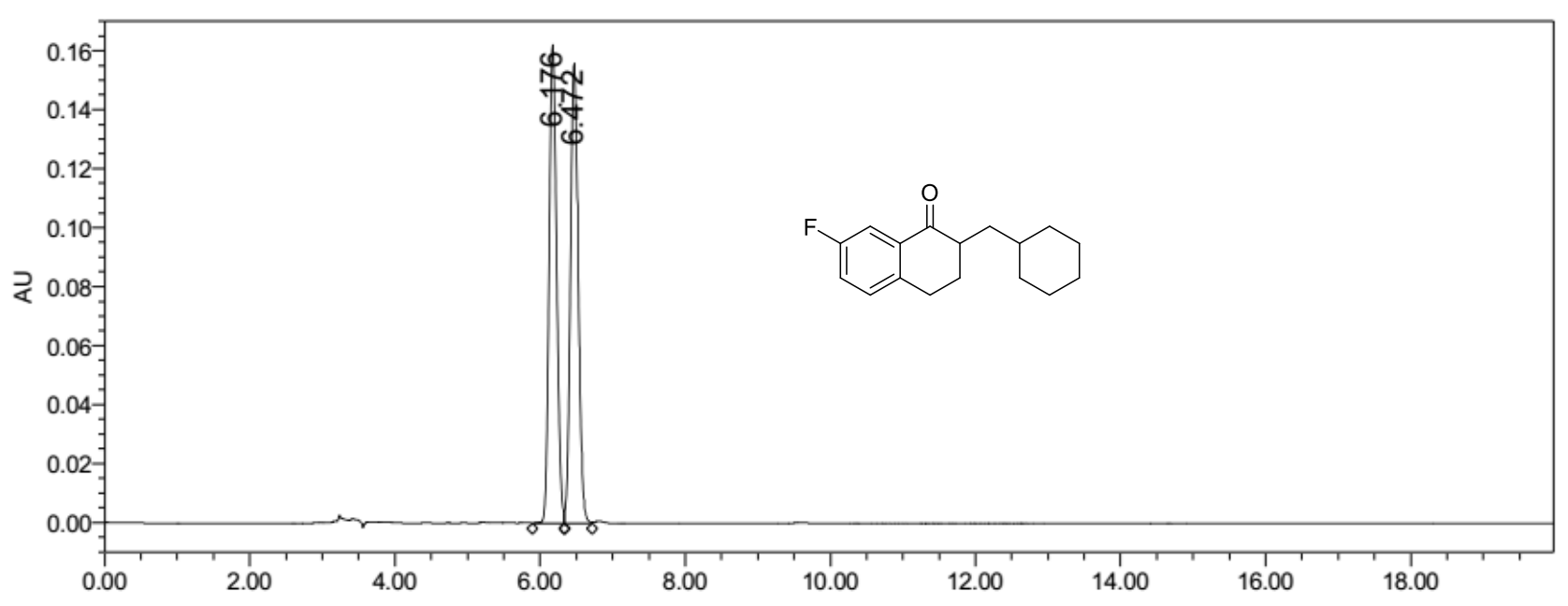

\begin{tabular}{|c|c|c|r|c|}
\hline & RT & Area & $\%$ Area & Height \\
\hline 1 & 6.176 & 1168830 & 49.96 & 162129 \\
\hline 2 & 6.472 & 1170826 & 50.04 & 155787 \\
\hline
\end{tabular}

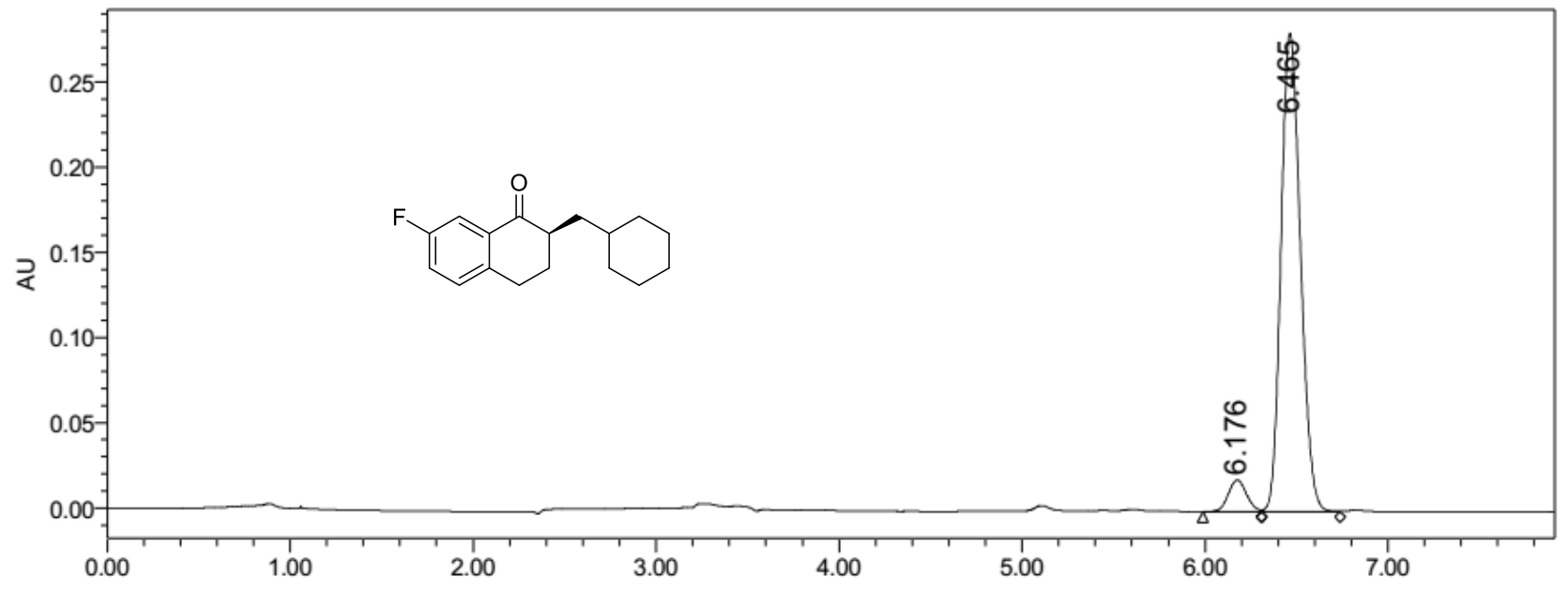

\begin{tabular}{|r|c|c|r|r|}
\hline & RT & Area & $\%$ Area & Height \\
\hline 1 & 6.176 & 128207 & 5.83 & 18533 \\
\hline 2 & 6.465 & 2071824 & 94.17 & 280572 \\
\hline
\end{tabular}




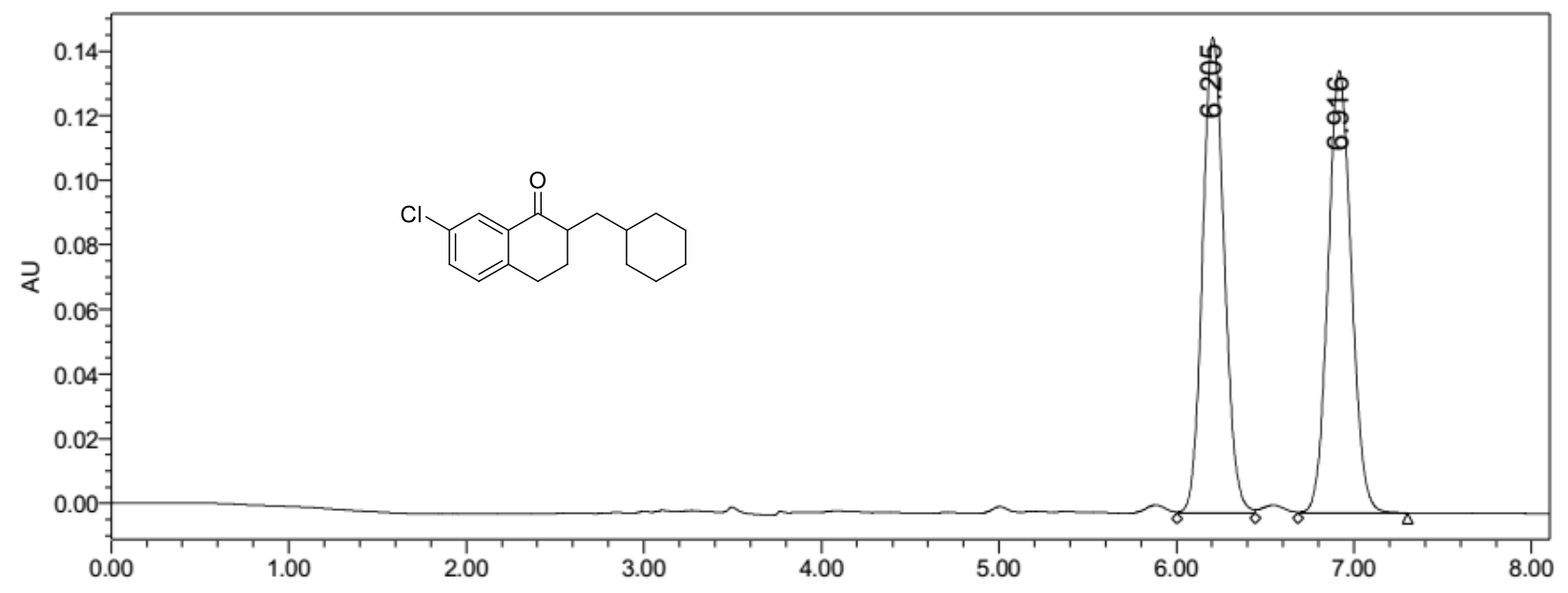

\begin{tabular}{|c|c|c|r|c|}
\hline & RT & Area & $\%$ Area & Height \\
\hline 1 & 6.205 & 1249876 & 49.97 & 147297 \\
\hline 2 & 6.916 & 1251300 & 50.03 & 136937 \\
\hline
\end{tabular}

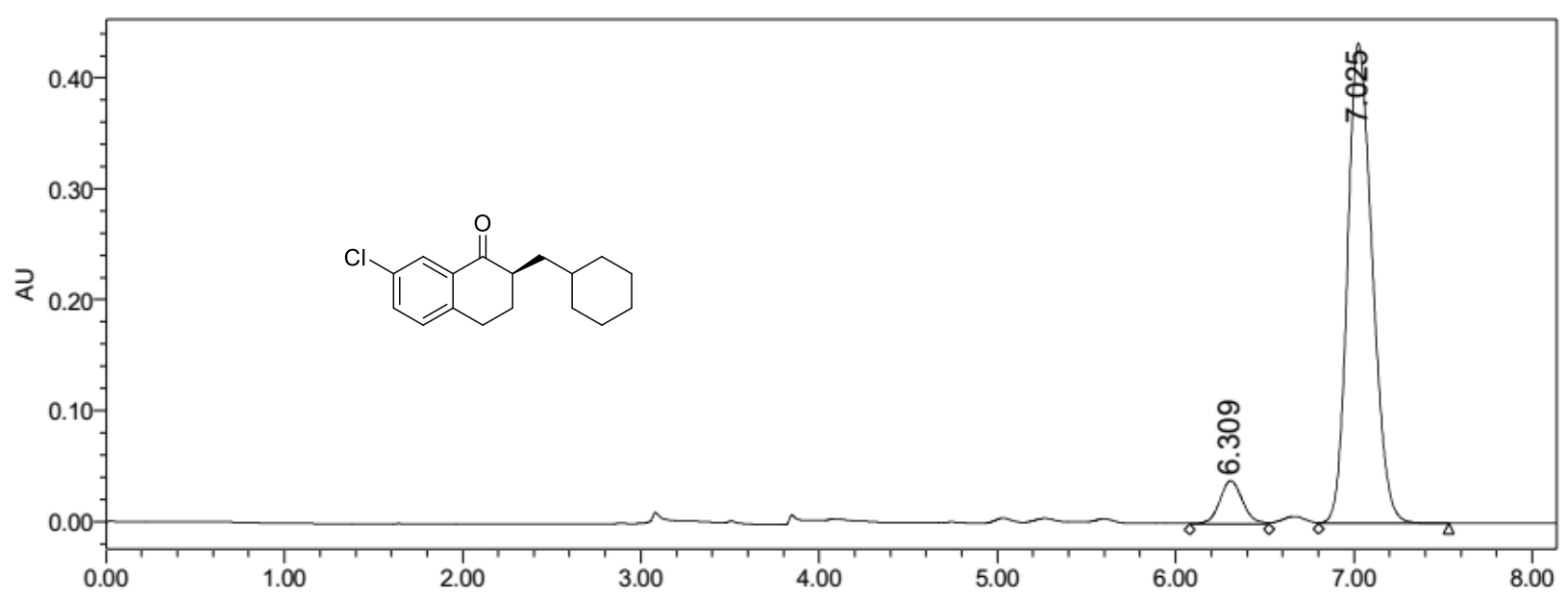

\begin{tabular}{|l|c|c|r|r|}
\hline & RT & \multicolumn{1}{c|}{ Area } & \% Area & Height \\
\hline 1 & 6.309 & 346567 & 7.63 & 38698 \\
\hline 2 & 7.025 & 4193874 & 92.37 & 432952 \\
\hline
\end{tabular}




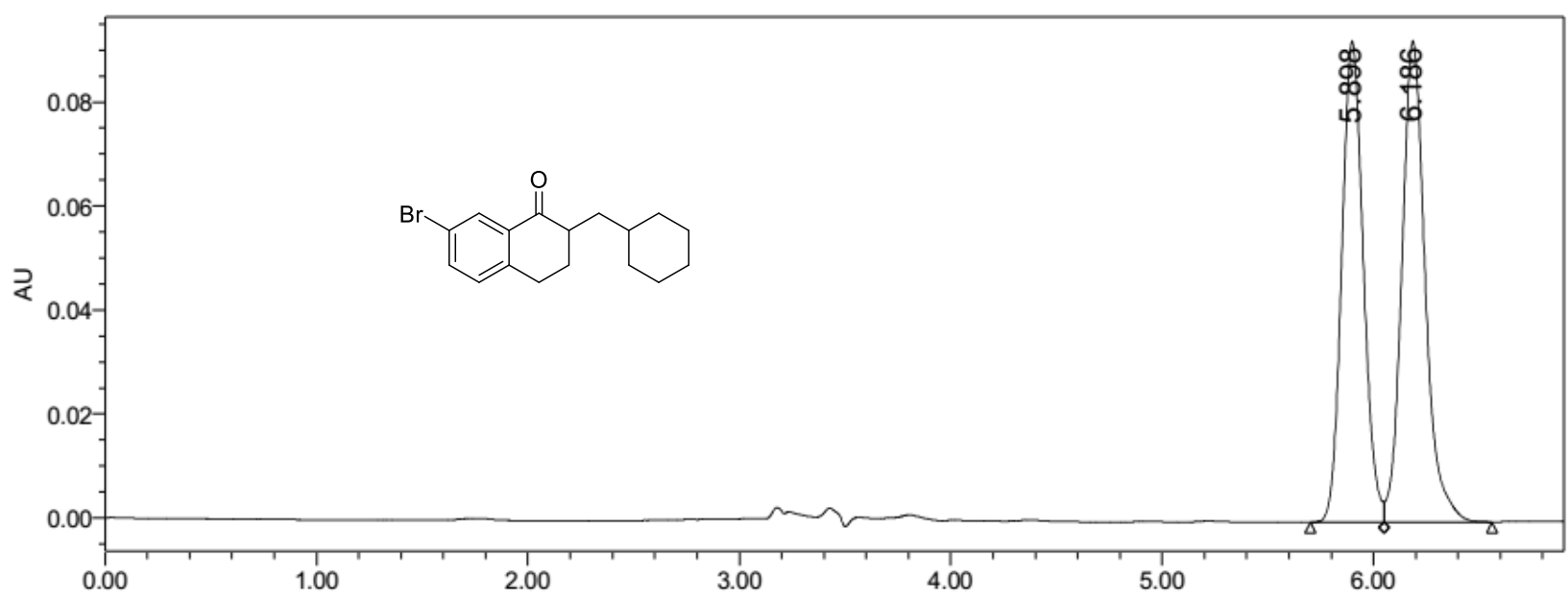

\begin{tabular}{|c|c|c|r|c|}
\hline & RT & Area & \% Area & Height \\
\hline 1 & 5.898 & 652590 & 47.96 & 92542 \\
\hline 2 & 6.186 & 708127 & 52.04 & 92576 \\
\hline
\end{tabular}

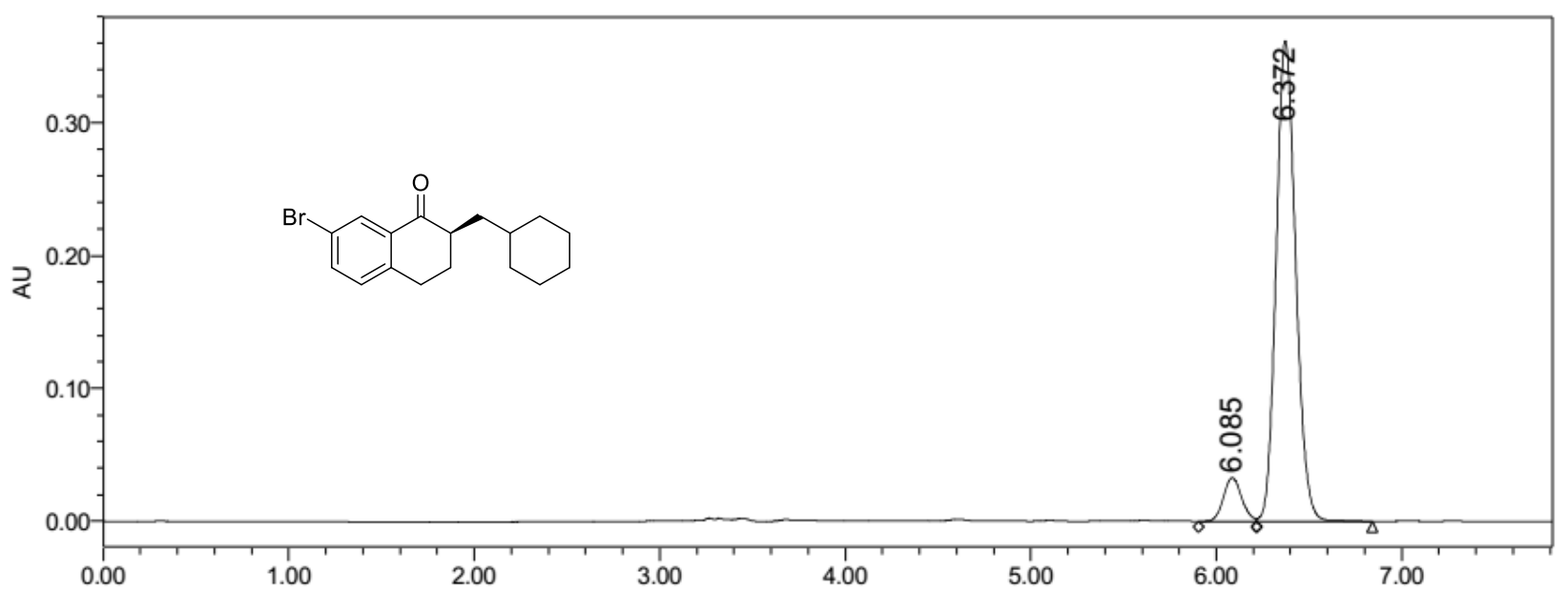

\begin{tabular}{|c|c|c|r|r|}
\hline & RT & Area & $\%$ Area & Height \\
\hline 1 & 6.085 & 225201 & 7.81 & 32897 \\
\hline 2 & 6.372 & 2659211 & 92.19 & 361805 \\
\hline
\end{tabular}




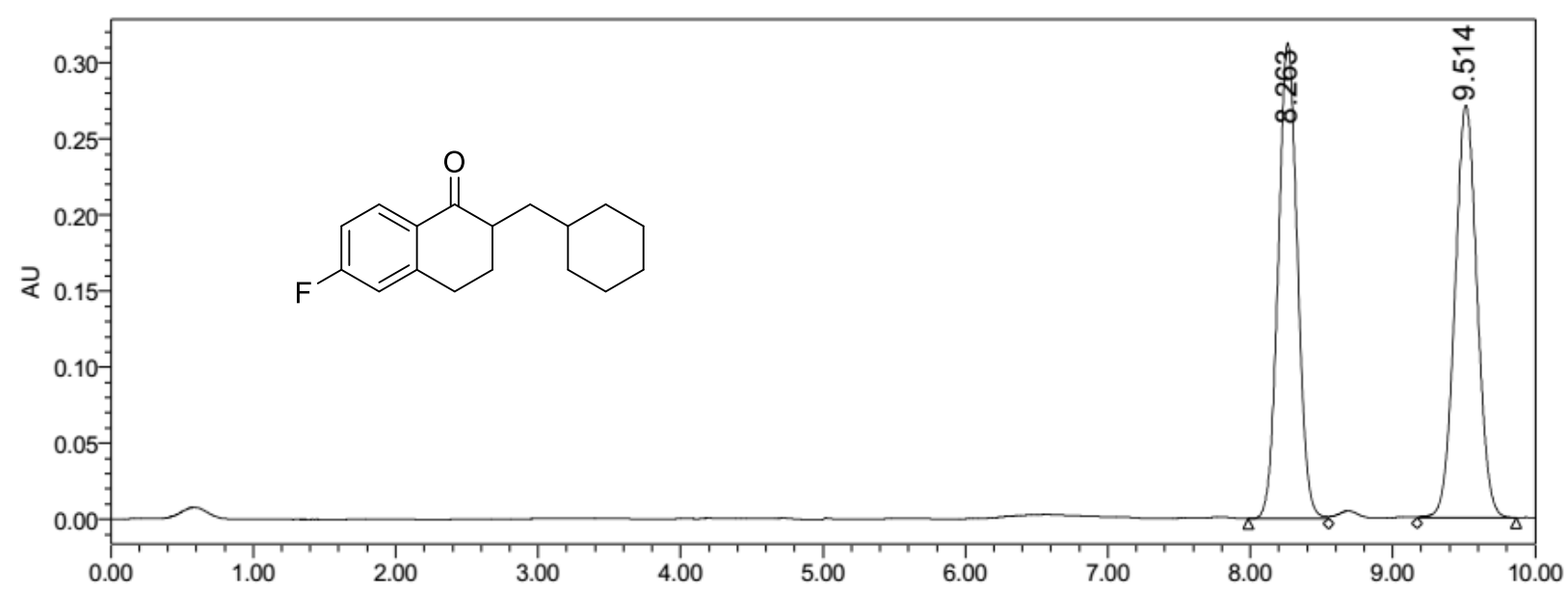

\begin{tabular}{|c|c|c|r|c|}
\hline & RT & Area & $\%$ Area & Height \\
\hline 1 & 8.263 & 2928807 & 49.90 & 312615 \\
\hline 2 & 9.514 & 2940127 & 50.10 & 271077 \\
\hline
\end{tabular}

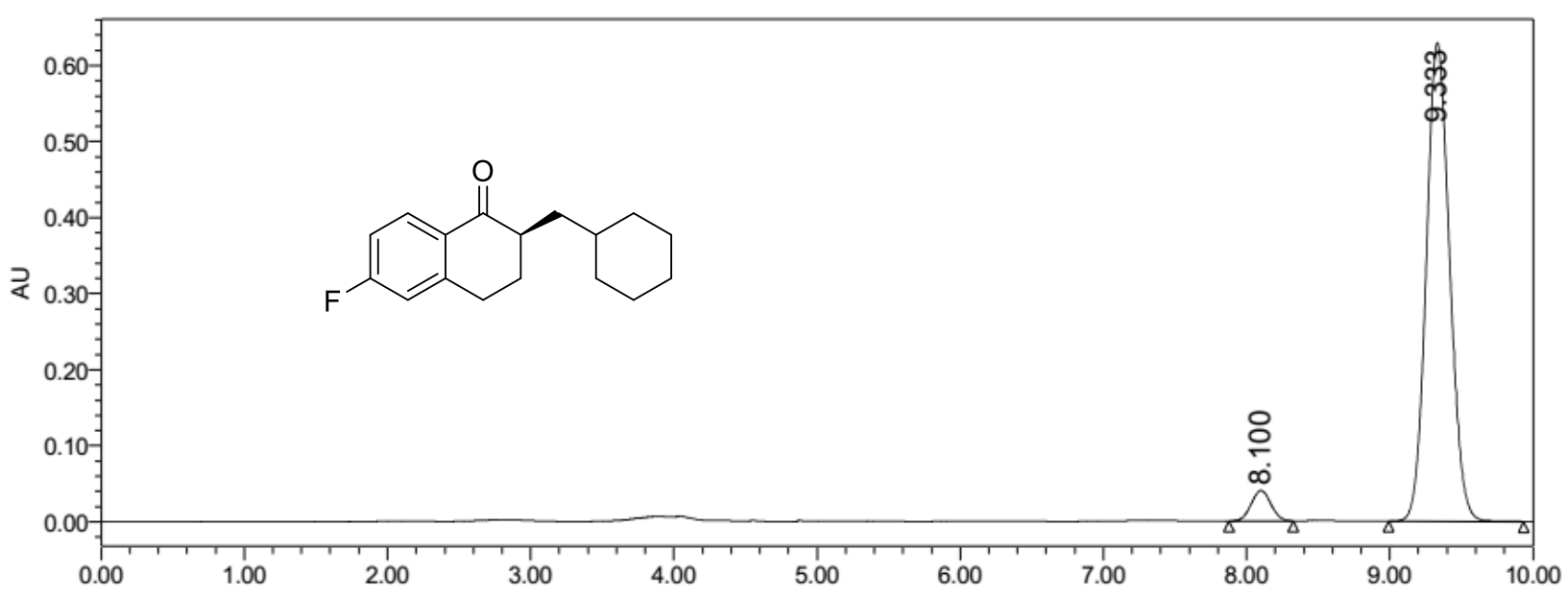

\begin{tabular}{|r|c|r|r|r|}
\hline & RT & Area & $\%$ Area & Height \\
\hline 1 & 8.100 & 387817 & 5.15 & 40242 \\
\hline 2 & 9.333 & 7146645 & 94.85 & 629424 \\
\hline
\end{tabular}




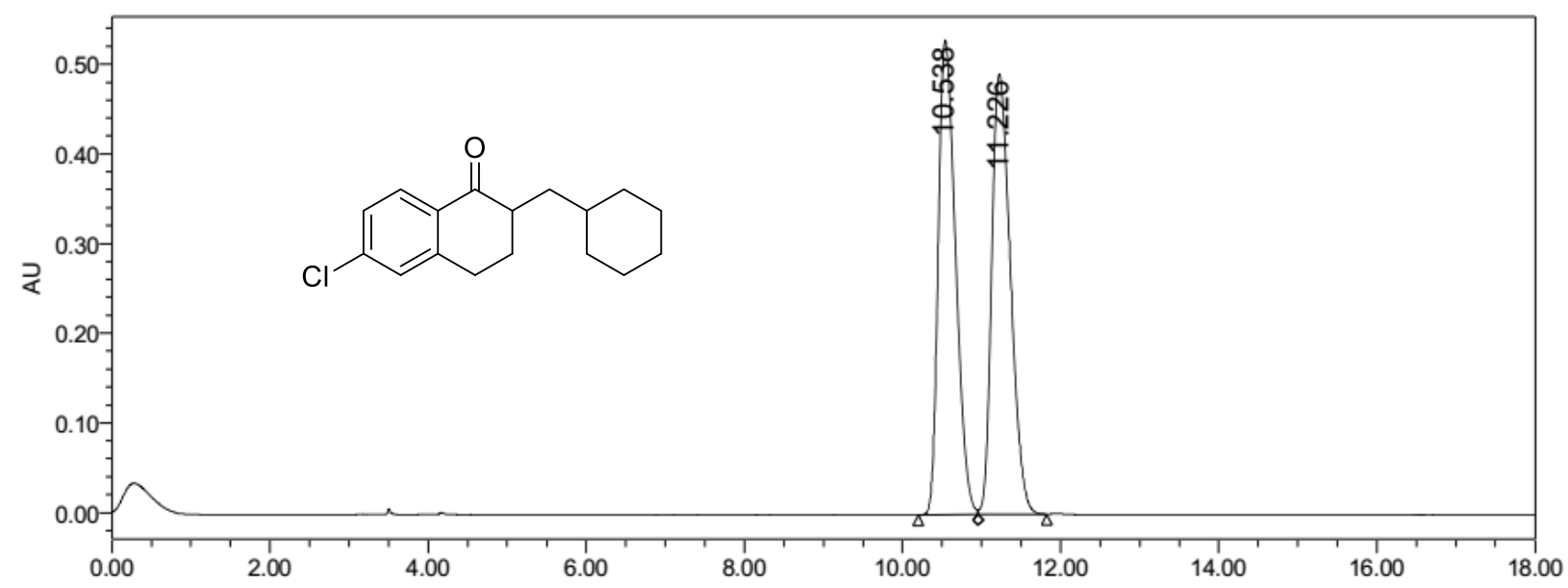

\begin{tabular}{|c|c|c|r|c|}
\hline & RT & Area & $\%$ Area & Height \\
\hline 1 & 10.538 & 8059278 & 49.92 & 529213 \\
\hline 2 & 11.226 & 8084648 & 50.08 & 490853 \\
\hline
\end{tabular}

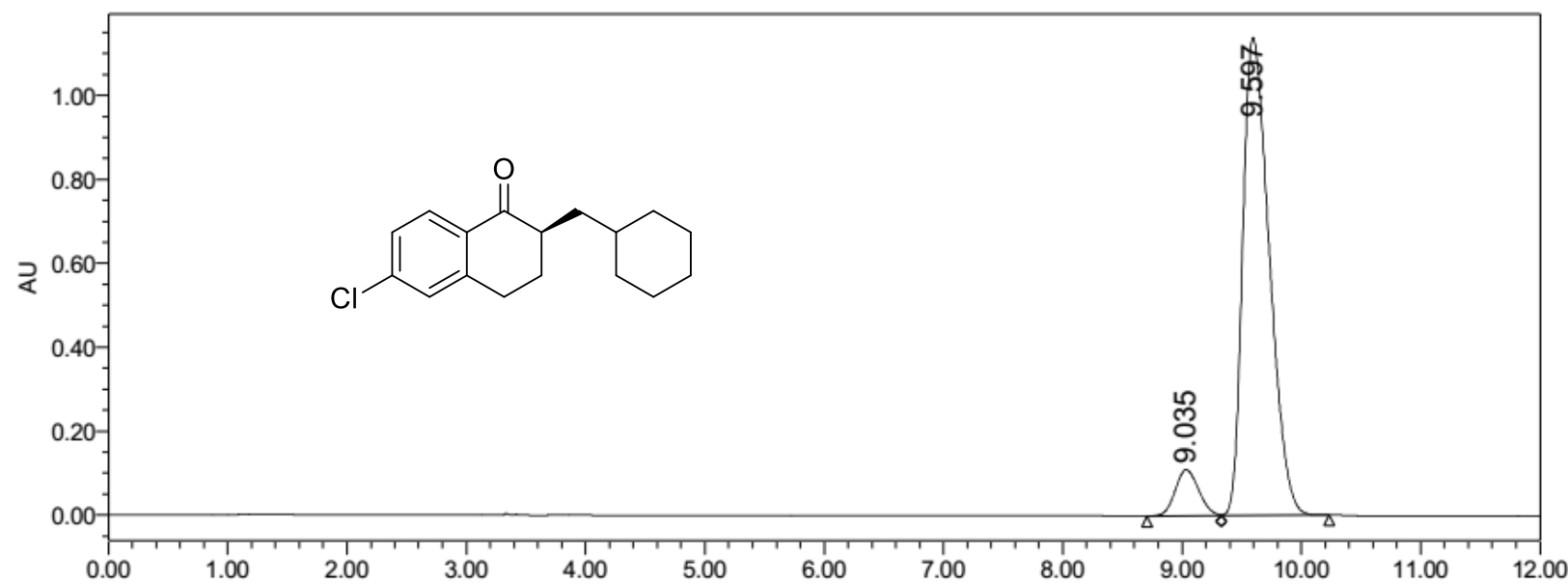

\begin{tabular}{|c|c|c|r|c|}
\hline & RT & Area & \% Area & Height \\
\hline 1 & 9.035 & 1503171 & 7.87 & 109594 \\
\hline 2 & 9.597 & 17593125 & 92.13 & 1138683 \\
\hline
\end{tabular}




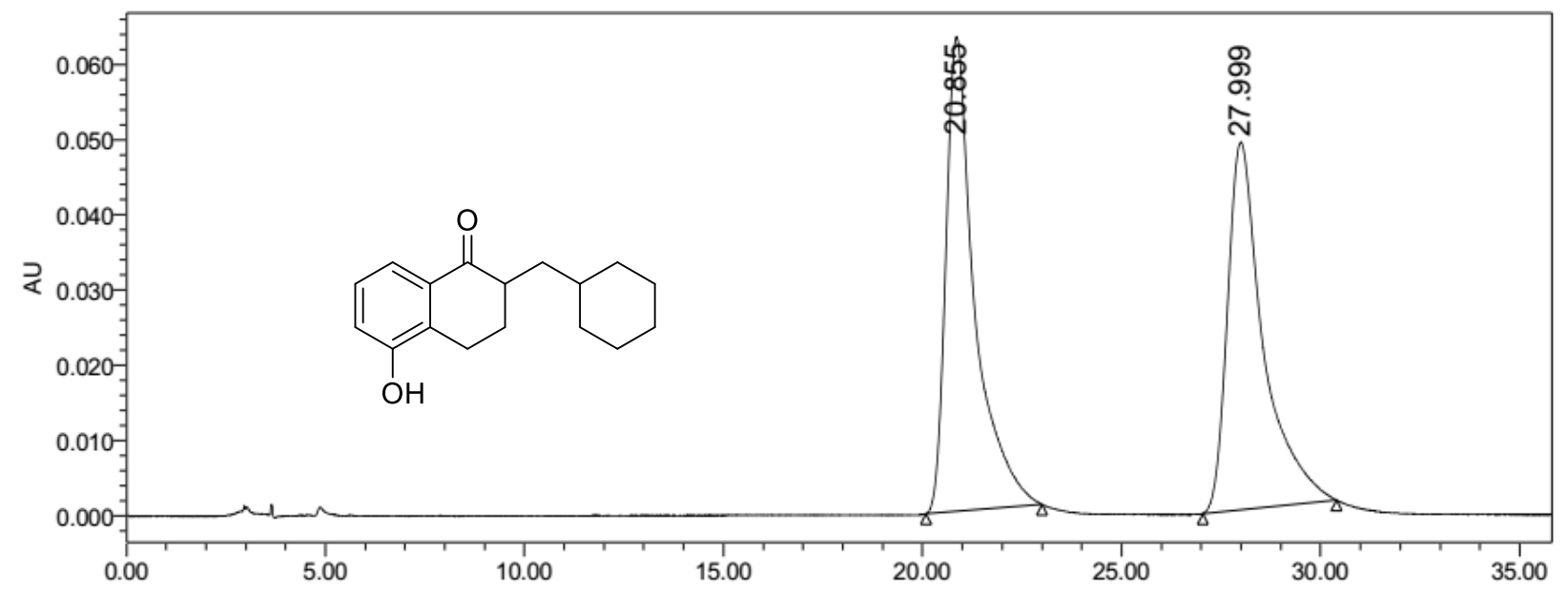

\begin{tabular}{|c|c|c|r|c|}
\hline & RT & Area & $\%$ Area & Height \\
\hline 1 & 20.855 & 3157765 & 50.89 & 63028 \\
\hline 2 & 27.999 & 3047802 & 49.11 & 48816 \\
\hline
\end{tabular}

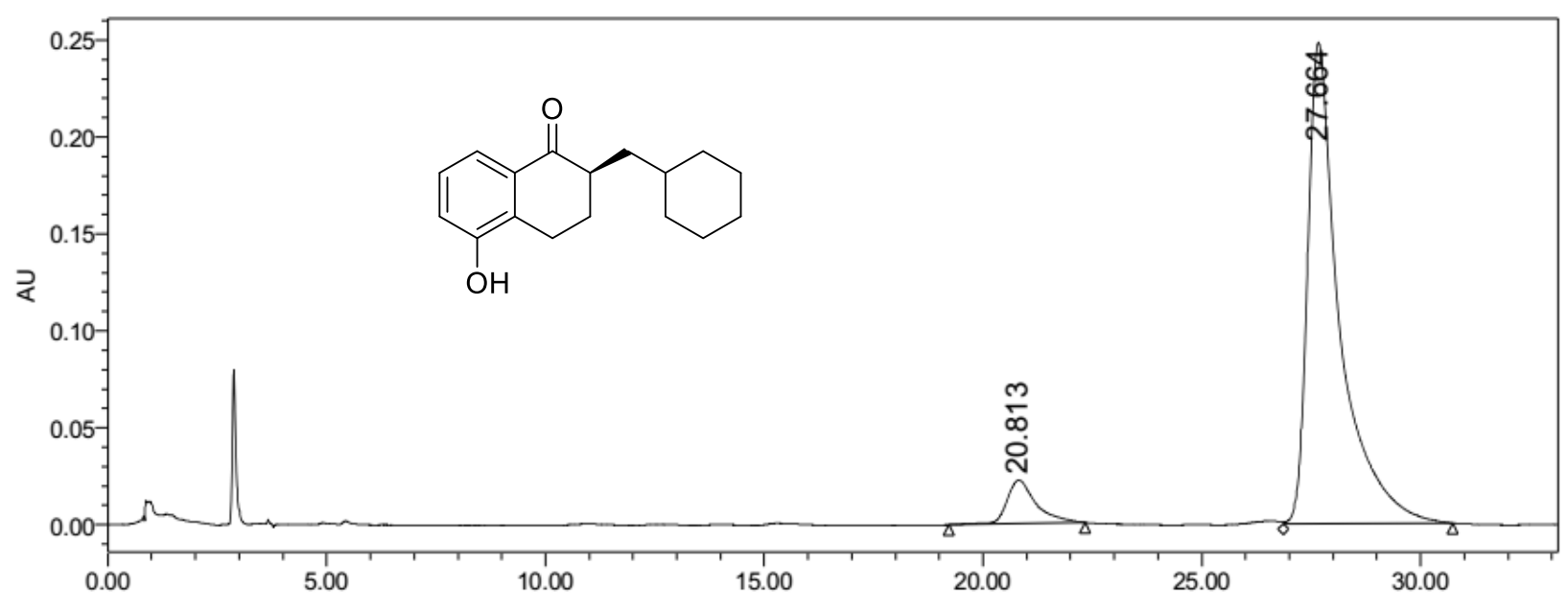

\begin{tabular}{|r|c|c|r|r|}
\hline & RT & Area & $\%$ Area & Height \\
\hline 1 & 20.813 & 1036460 & 7.58 & 22378 \\
\hline 2 & 27.664 & 12637176 & 92.42 & 248080 \\
\hline
\end{tabular}




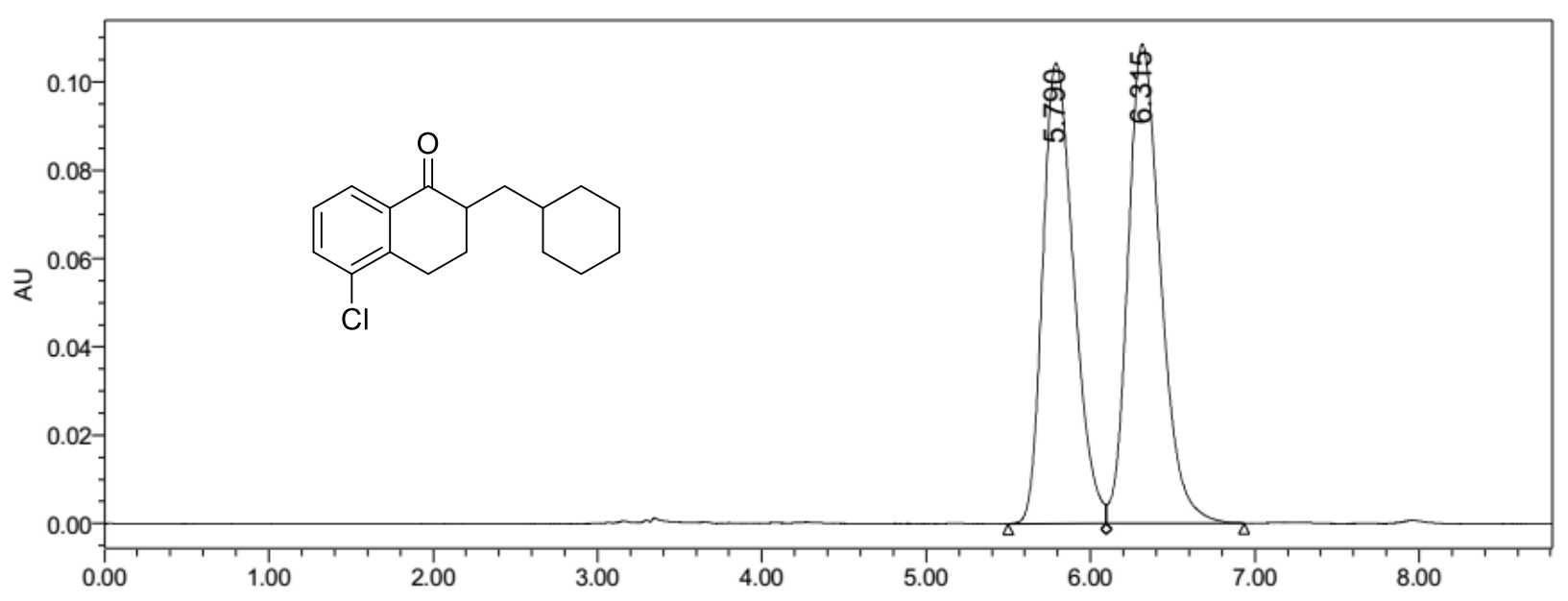

\begin{tabular}{|c|c|c|r|c|}
\hline & RT & Area & $\%$ Area & Height \\
\hline 1 & 5.790 & 1365040 & 47.65 & 104350 \\
\hline 2 & 6.315 & 1499908 & 52.35 & 108540 \\
\hline
\end{tabular}

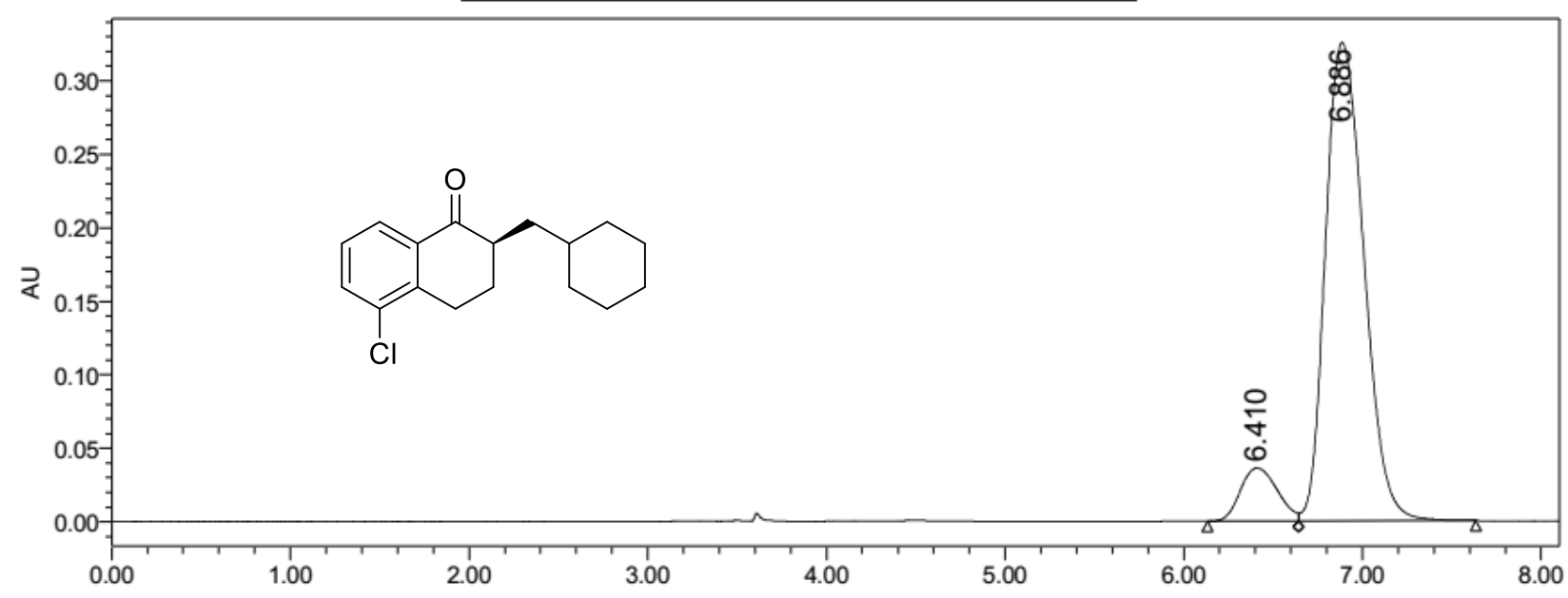

\begin{tabular}{|r|c|r|r|r|}
\hline & RT & \multicolumn{1}{|c|}{ Area } & $\%$ Area & Height \\
\hline 1 & 6.410 & 521364 & 9.68 & 36218 \\
\hline 2 & 6.886 & 4864750 & 90.32 & 325645 \\
\hline
\end{tabular}



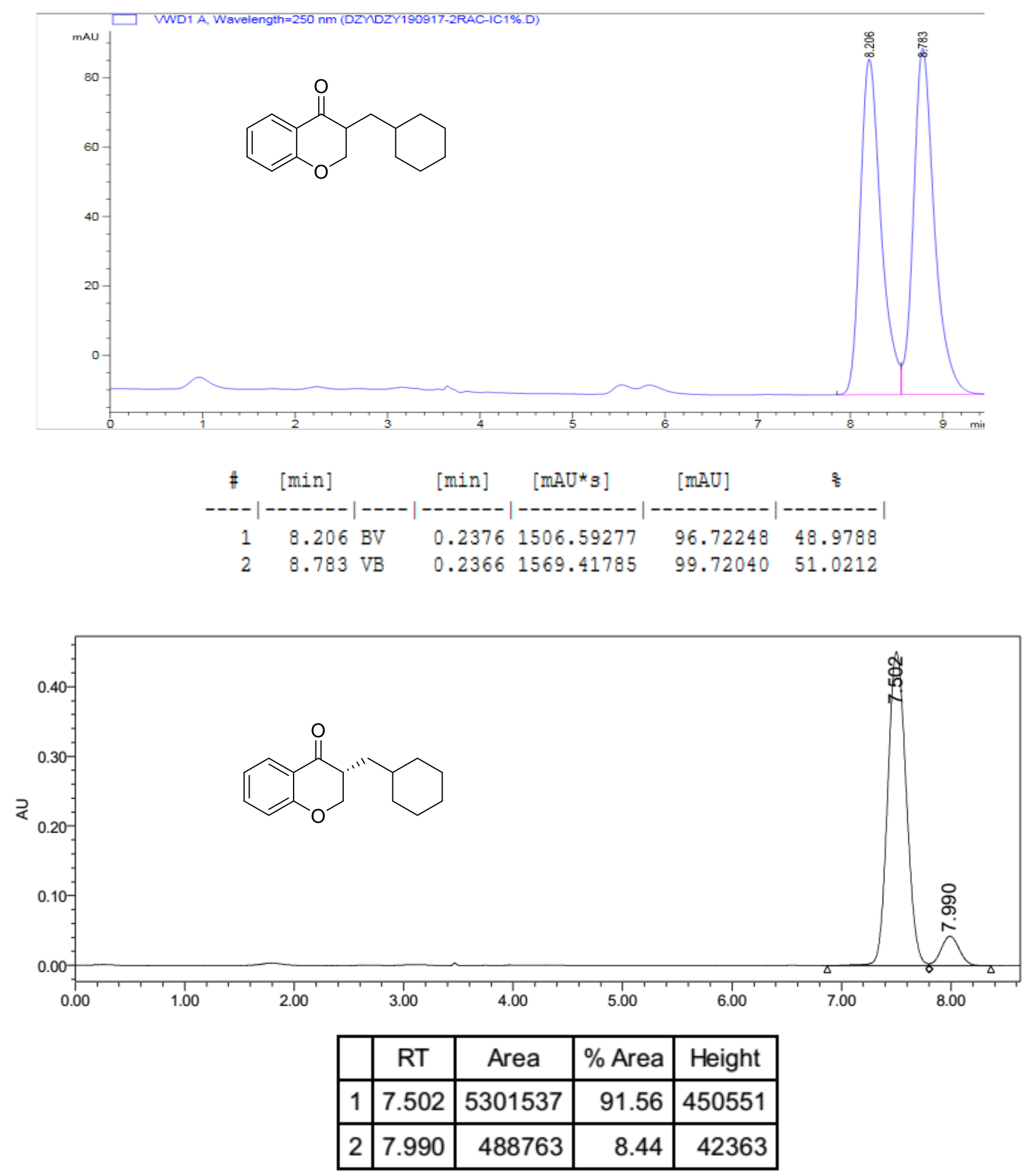

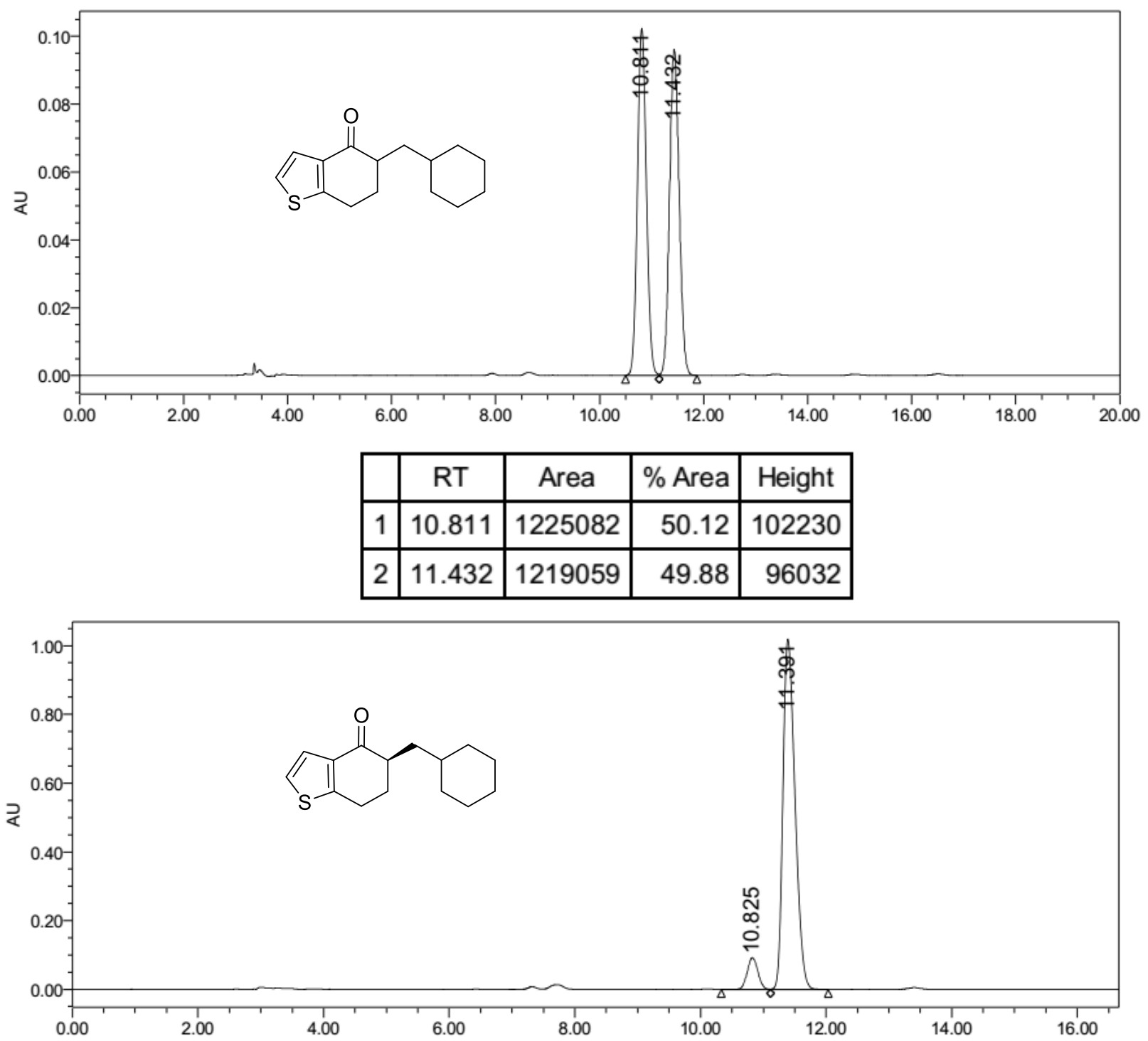

\begin{tabular}{|r|c|c|r|r|}
\hline & RT & Area & \% Area & Height \\
\hline 1 & 10.825 & 1091558 & 7.40 & 92565 \\
\hline 2 & 11.391 & 13653157 & 92.60 & 1019327 \\
\hline
\end{tabular}




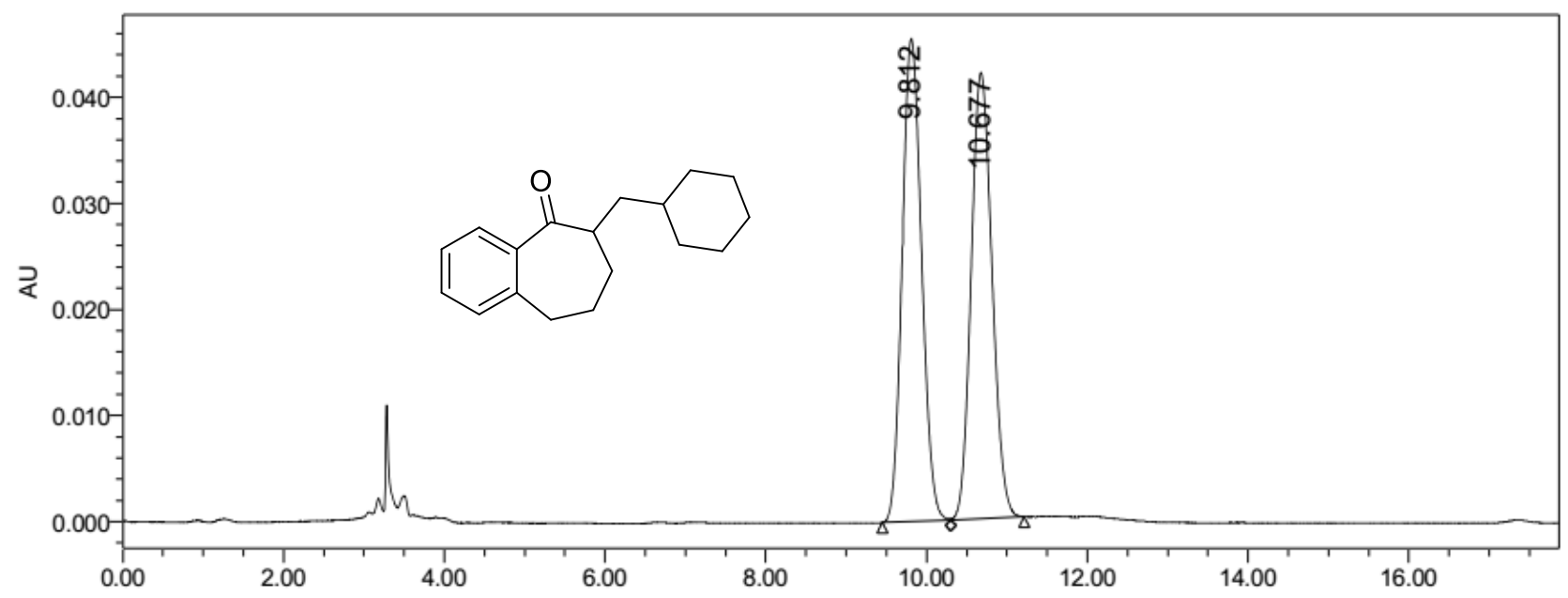

\begin{tabular}{|c|c|c|r|c|}
\hline & RT & Area & $\%$ Area & Height \\
\hline 1 & 9.812 & 761108 & 50.23 & 45491 \\
\hline 2 & 10.677 & 754082 & 49.77 & 42054 \\
\hline
\end{tabular}

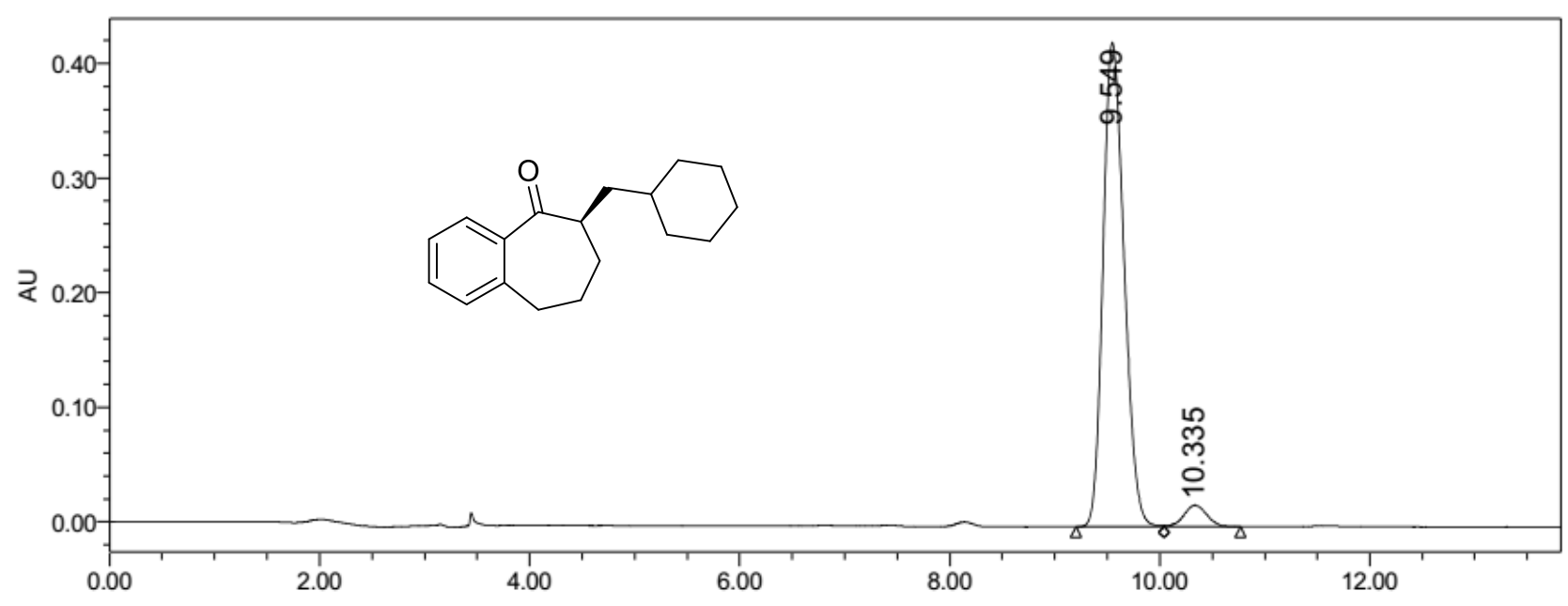

\begin{tabular}{|l|c|r|r|r|}
\hline & \multicolumn{1}{|c|}{ RT } & Area & \% Area & Height \\
\hline 1 & 9.549 & 5918755 & 95.32 & 422643 \\
\hline 2 & 10.335 & 290790 & 4.68 & 18843 \\
\hline
\end{tabular}




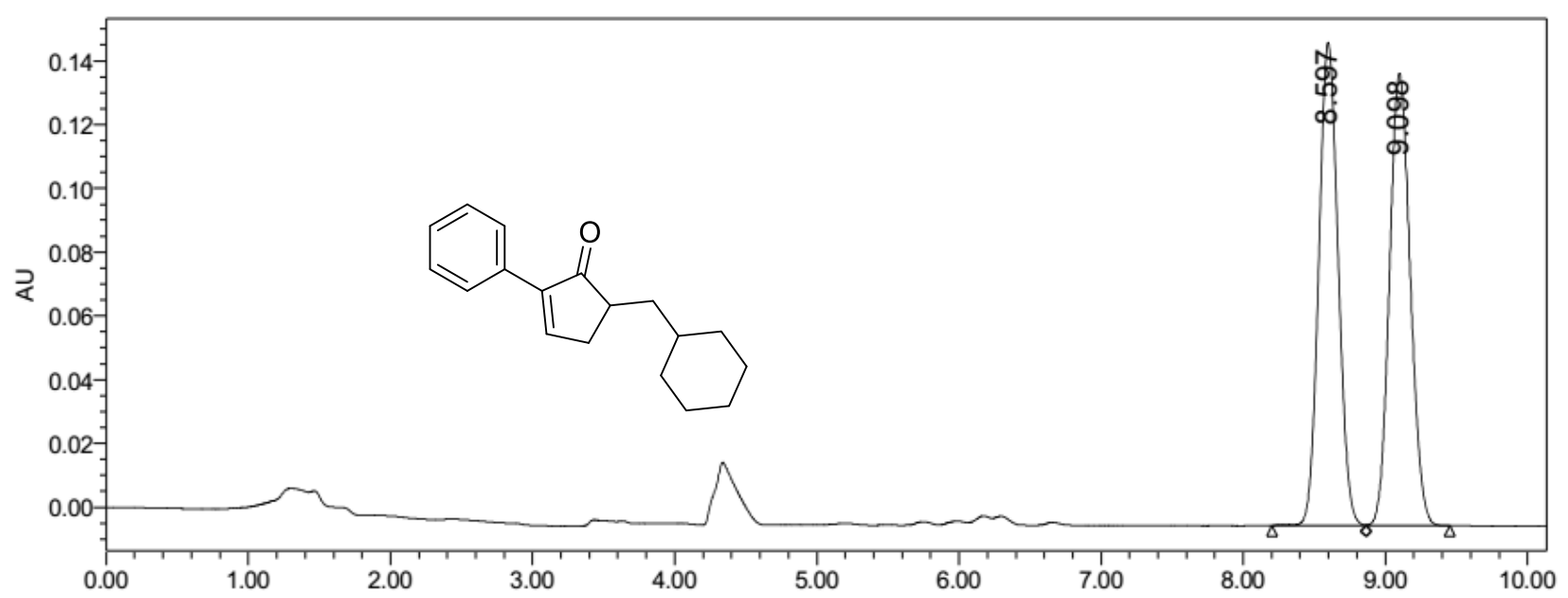

\begin{tabular}{|c|c|c|r|c|}
\hline & RT & Area & $\%$ Area & Height \\
\hline 1 & 8.597 & 1440897 & 50.04 & 151506 \\
\hline 2 & 9.098 & 1438773 & 49.96 & 141850 \\
\hline
\end{tabular}

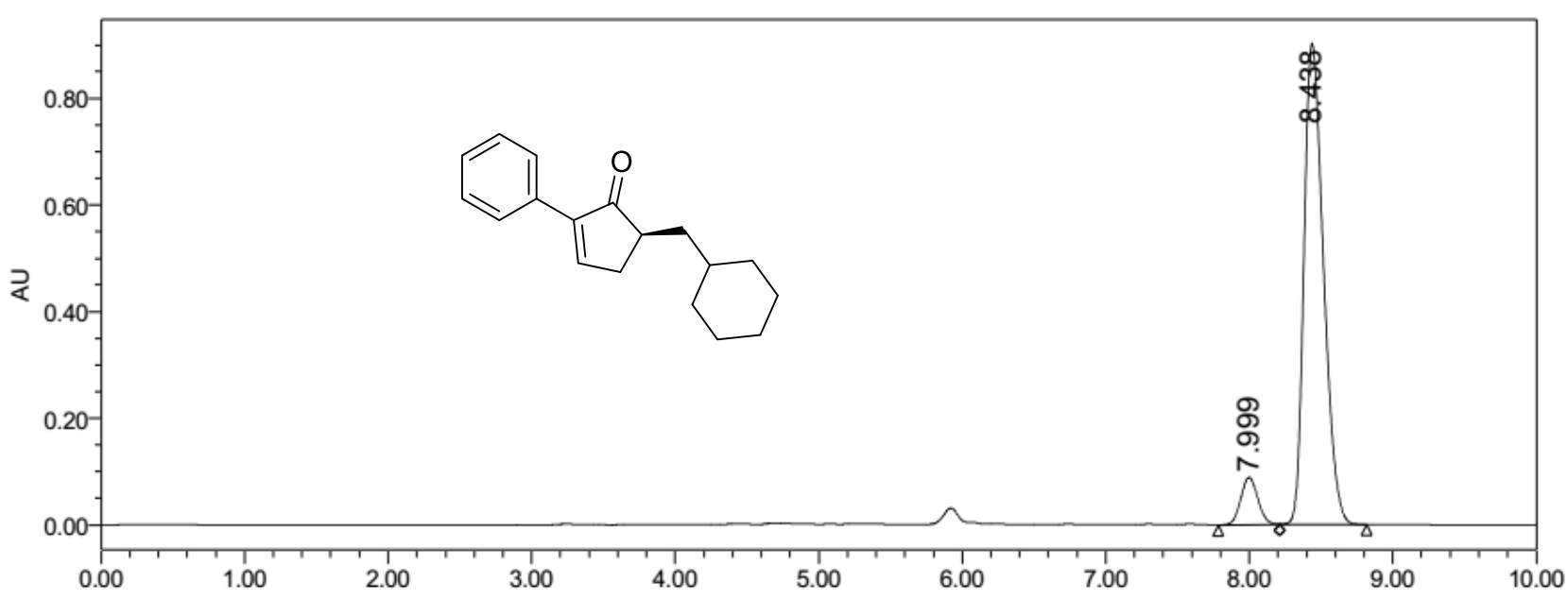

\begin{tabular}{|c|c|c|r|r|}
\hline & RT & Area & $\%$ Area & Height \\
\hline 1 & 7.999 & 743917 & 8.02 & 89051 \\
\hline 2 & 8.438 & 8536639 & 91.98 & 902325 \\
\hline
\end{tabular}




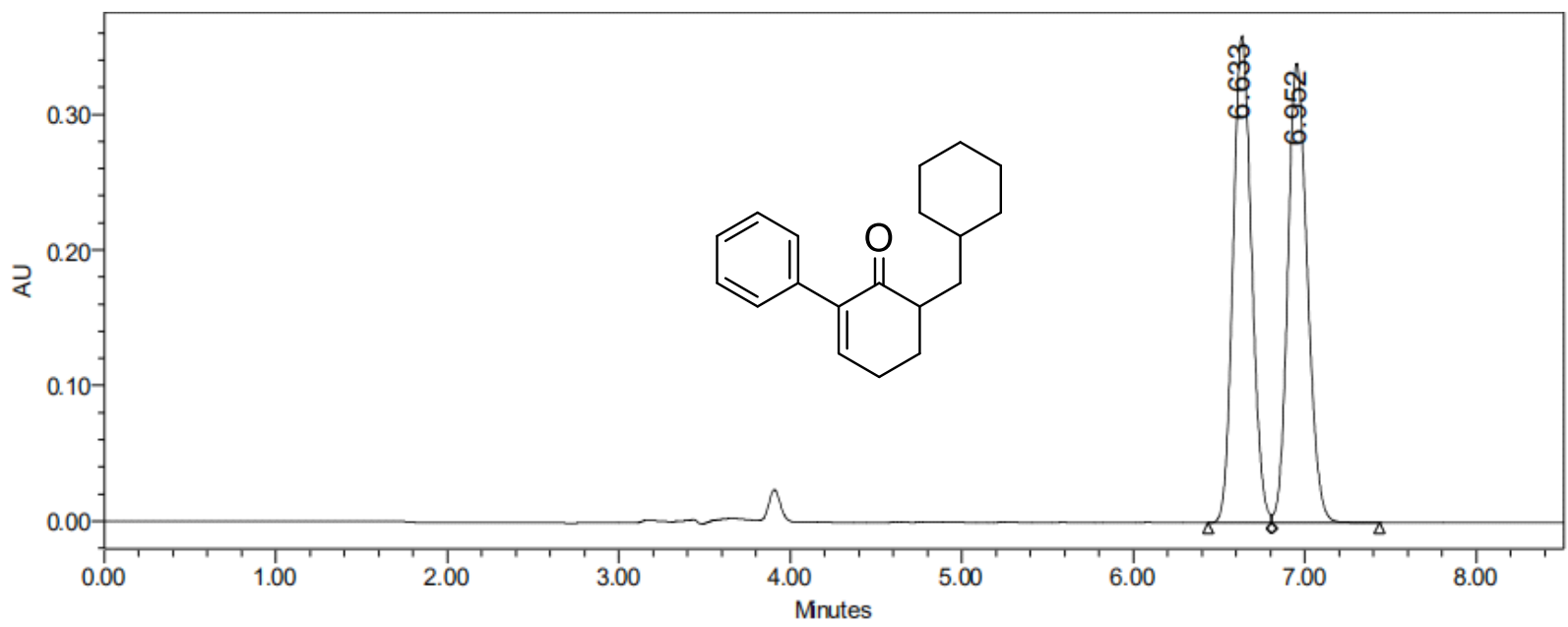

\begin{tabular}{|c|c|c|r|c|}
\hline & RT & Area & \% Area & Height \\
\hline 1 & 6.633 & 2689906 & 49.86 & 358653 \\
\hline 2 & 6.952 & 2704927 & 50.14 & 338592 \\
\hline
\end{tabular}

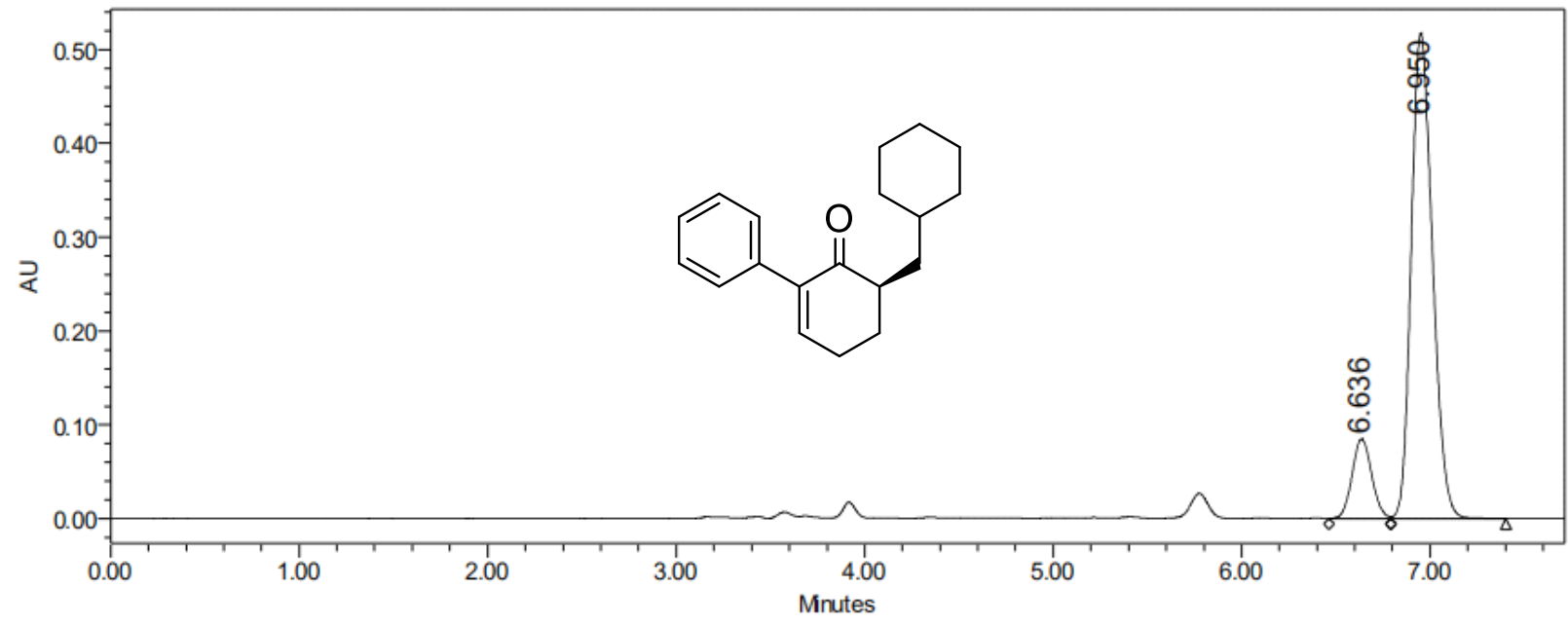

\begin{tabular}{|r|c|c|r|r|}
\hline & RT & \multicolumn{1}{|c|}{ Area } & $\%$ Area & Height \\
\hline 1 & 6.636 & 618084 & 13.26 & 84983 \\
\hline 2 & 6.950 & 4041796 & 86.74 & 517231 \\
\hline
\end{tabular}




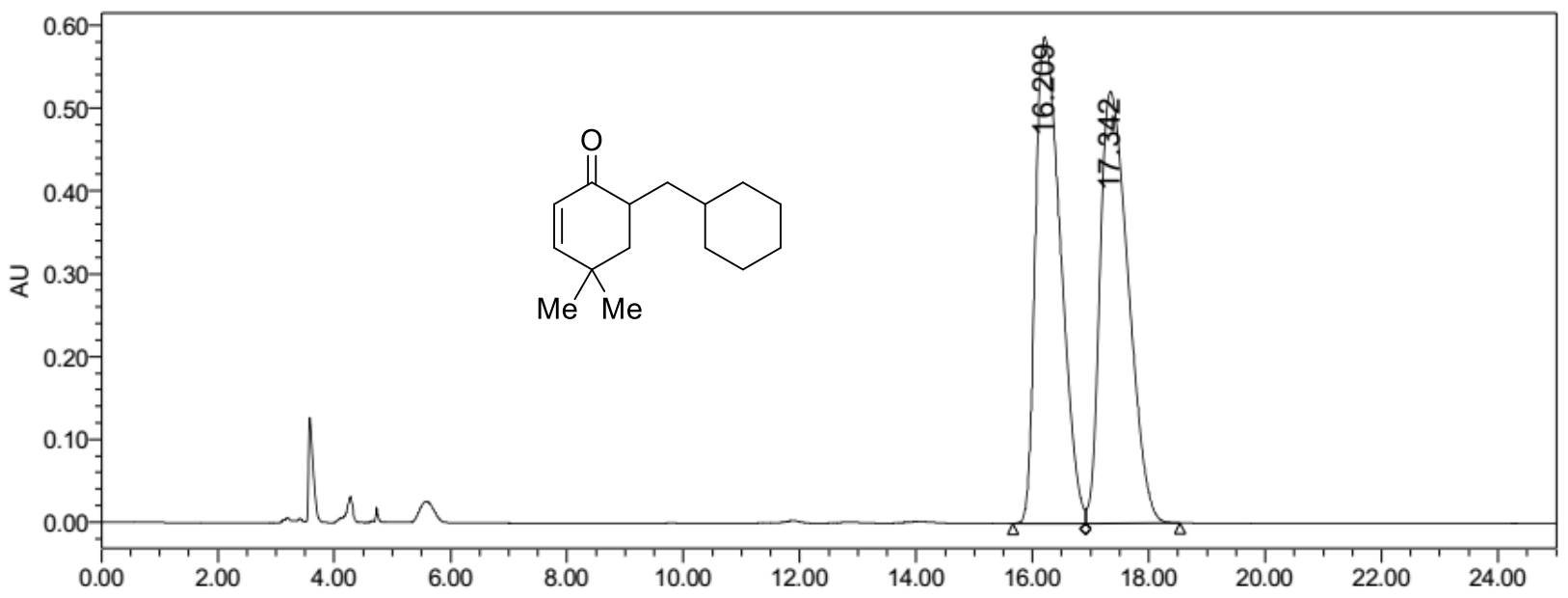

\begin{tabular}{|c|c|c|r|c|}
\hline & RT & Area & $\%$ Area & Height \\
\hline 1 & 16.209 & 17498093 & 49.75 & 587183 \\
\hline 2 & 17.342 & 17674859 & 50.25 & 521113 \\
\hline
\end{tabular}

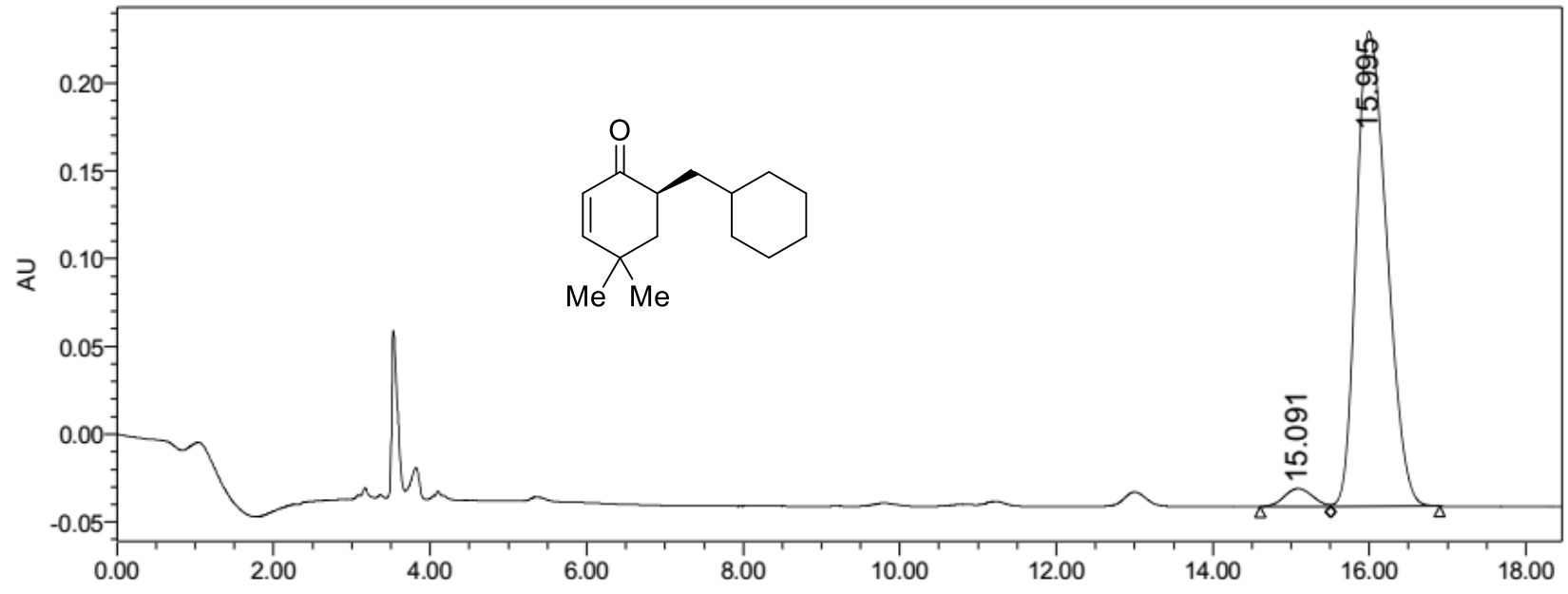

\begin{tabular}{|c|c|c|r|r|}
\hline & RT & Area & $\%$ Area & Height \\
\hline 1 & 15.091 & 249639 & 3.30 & 10137 \\
\hline 2 & 15.995 & 7315520 & 96.70 & 270541 \\
\hline
\end{tabular}



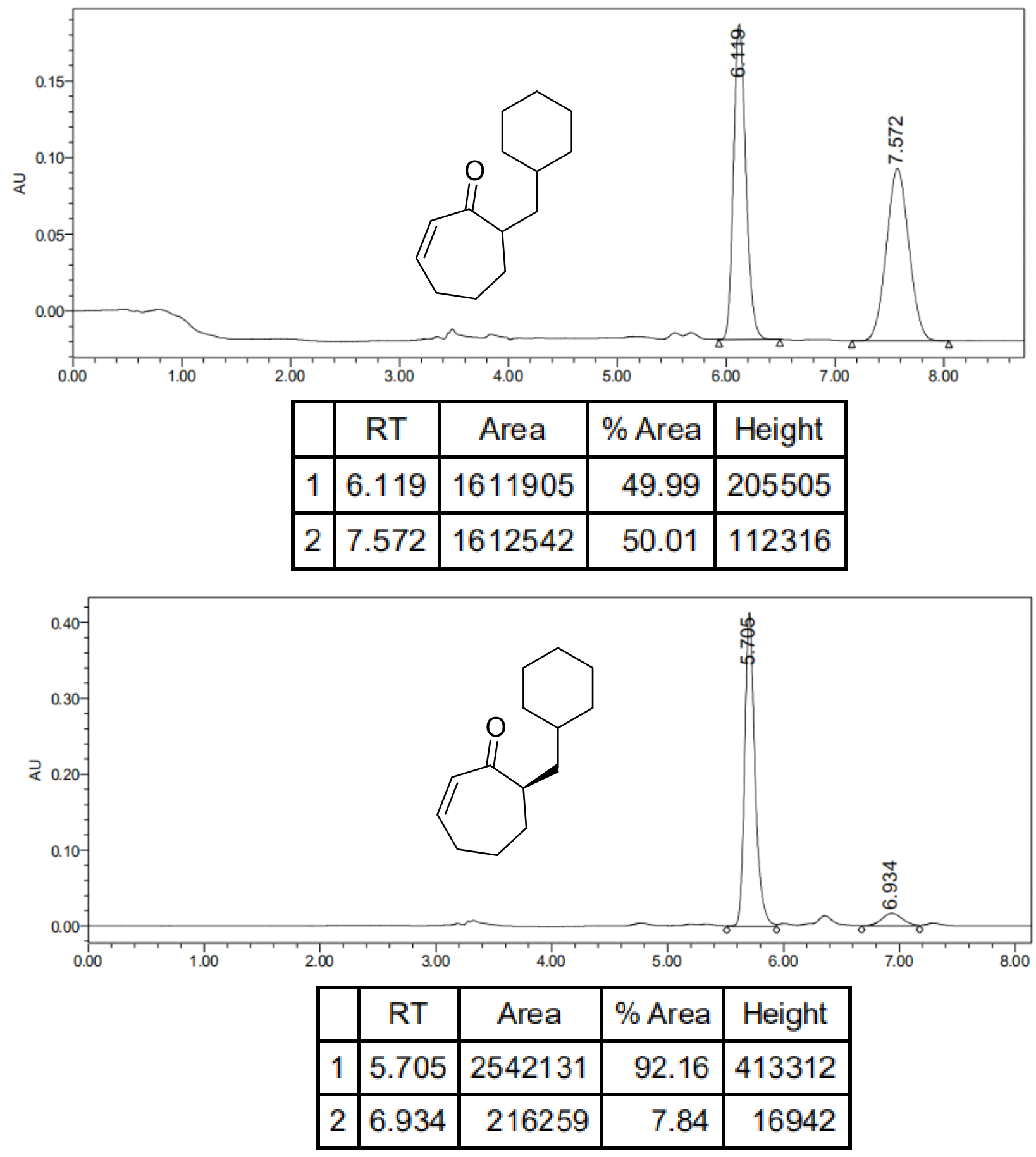


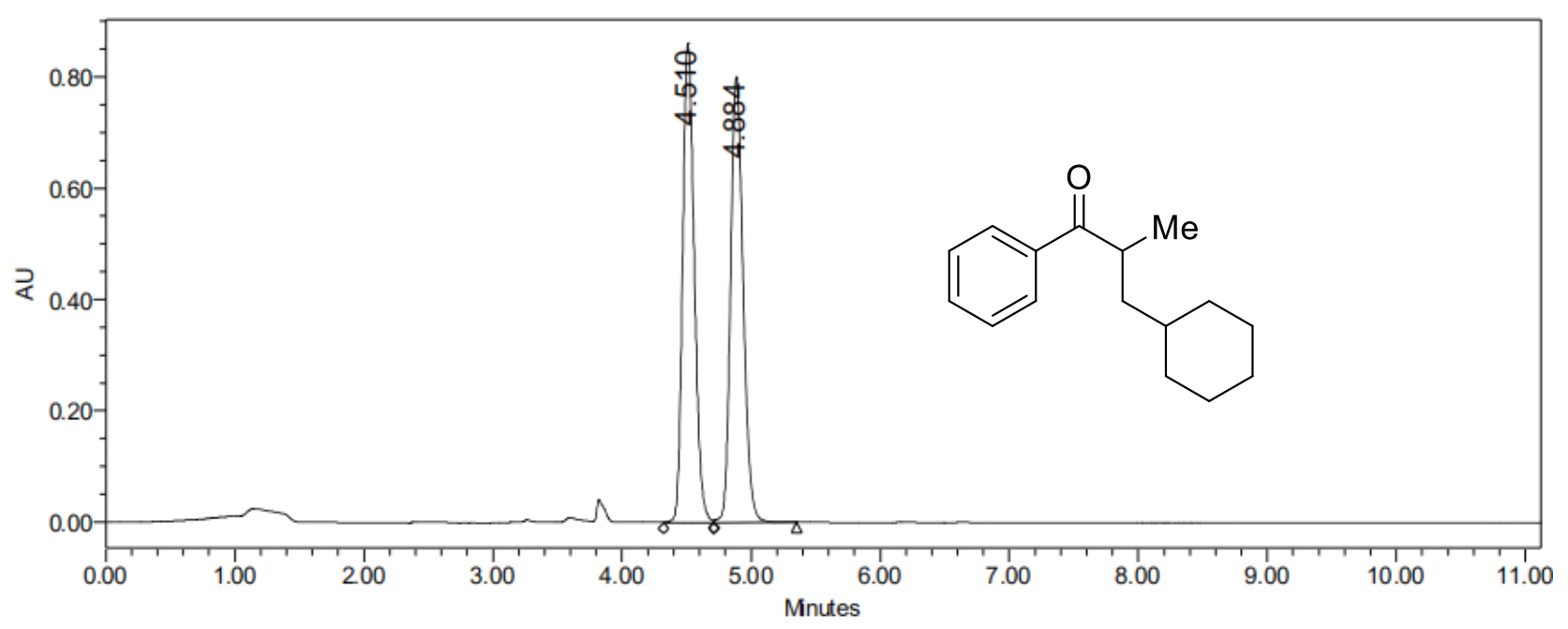

\begin{tabular}{|c|c|c|r|c|}
\hline & RT & Area & \% Area & Height \\
\hline 1 & 4.510 & 5428358 & 49.92 & 861169 \\
\hline 2 & 4.884 & 5446777 & 50.08 & 800413 \\
\hline
\end{tabular}

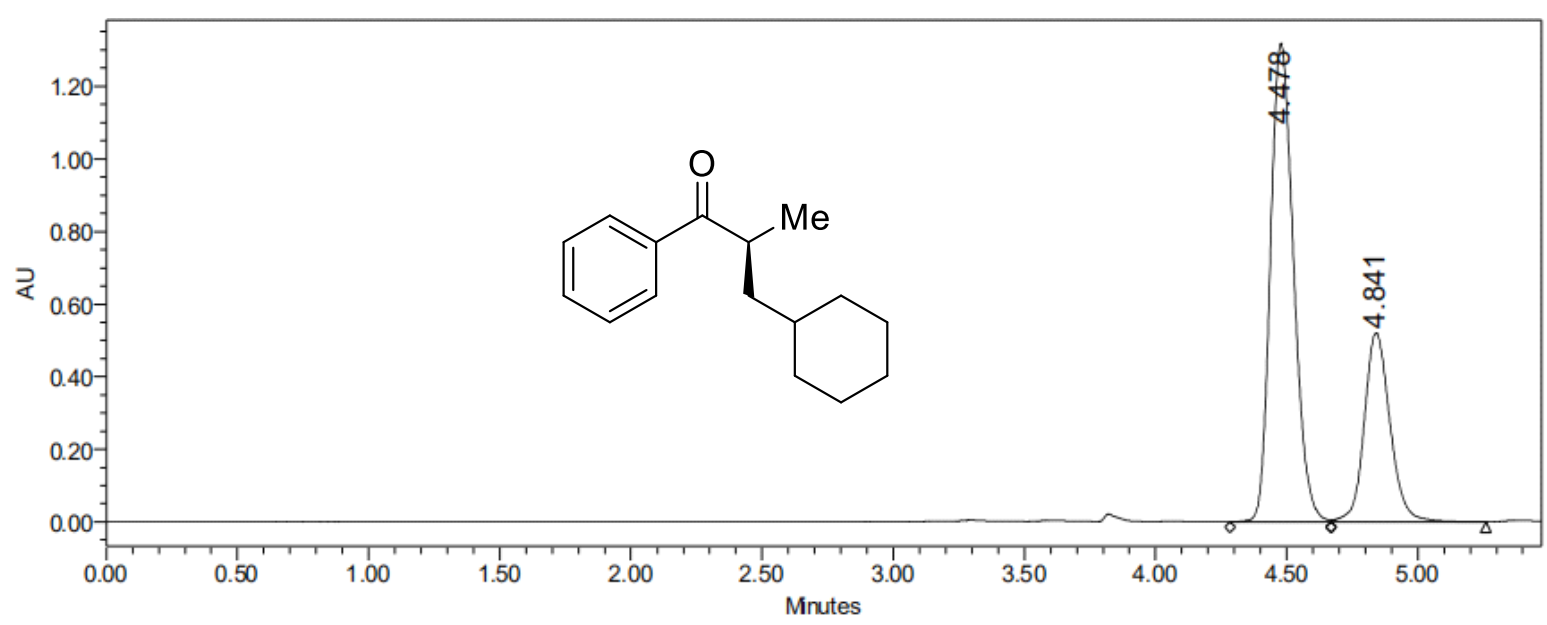

\begin{tabular}{|c|c|c|r|c|}
\hline & RT & Area & $\%$ Area & Height \\
\hline 1 & 4.478 & 8078007 & 69.40 & 1316984 \\
\hline 2 & 4.841 & 3562460 & 30.60 & 519965 \\
\hline
\end{tabular}




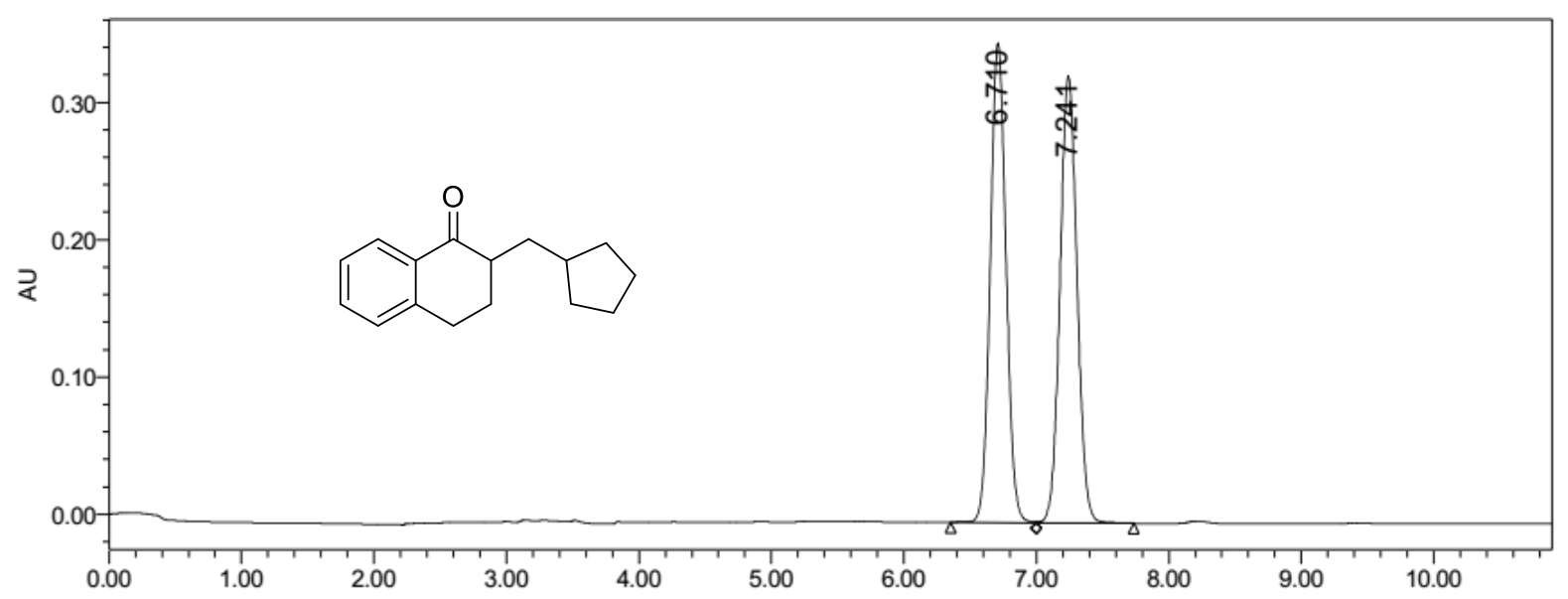

\begin{tabular}{|c|c|c|r|c|}
\hline & RT & Area & $\%$ Area & Height \\
\hline 1 & 6.710 & 2900509 & 49.96 & 348987 \\
\hline 2 & 7.241 & 2905190 & 50.04 & 325361 \\
\hline
\end{tabular}

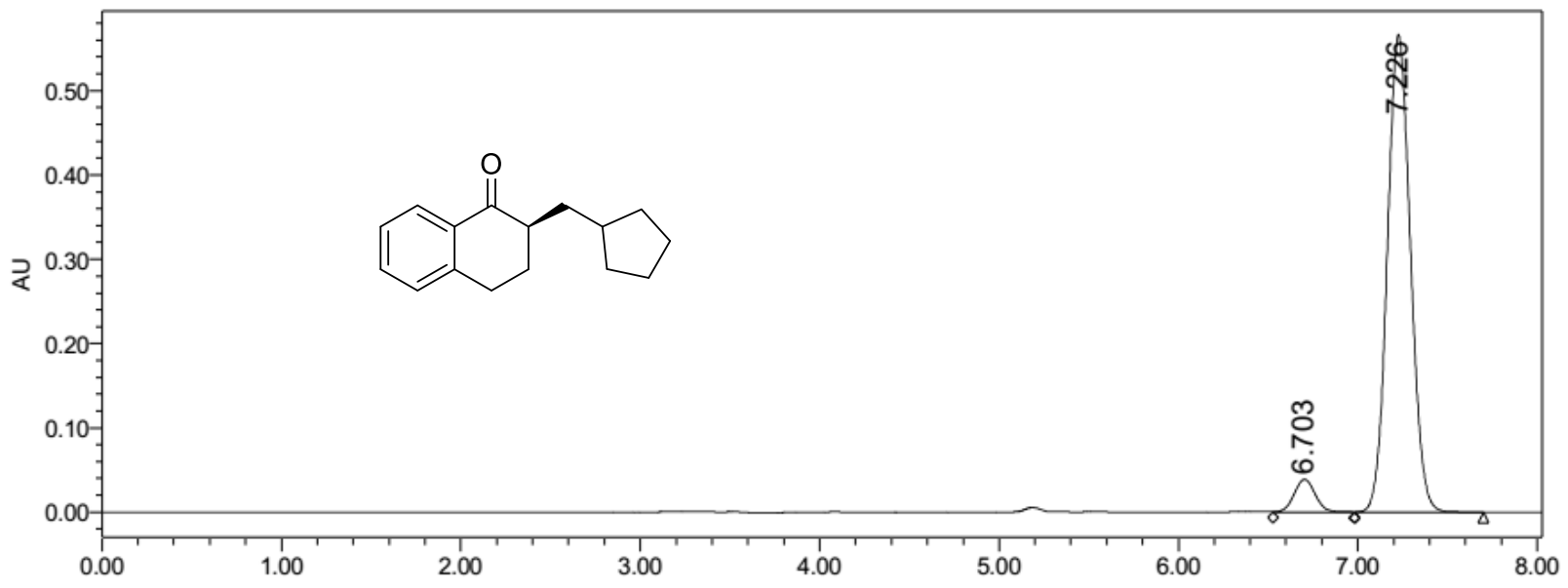

\begin{tabular}{|c|c|c|r|c|}
\hline & RT & Area & $\%$ Area & Height \\
\hline 1 & 6.703 & 323492 & 6.00 & 39063 \\
\hline 2 & 7.226 & 5066247 & 94.00 & 566611 \\
\hline
\end{tabular}




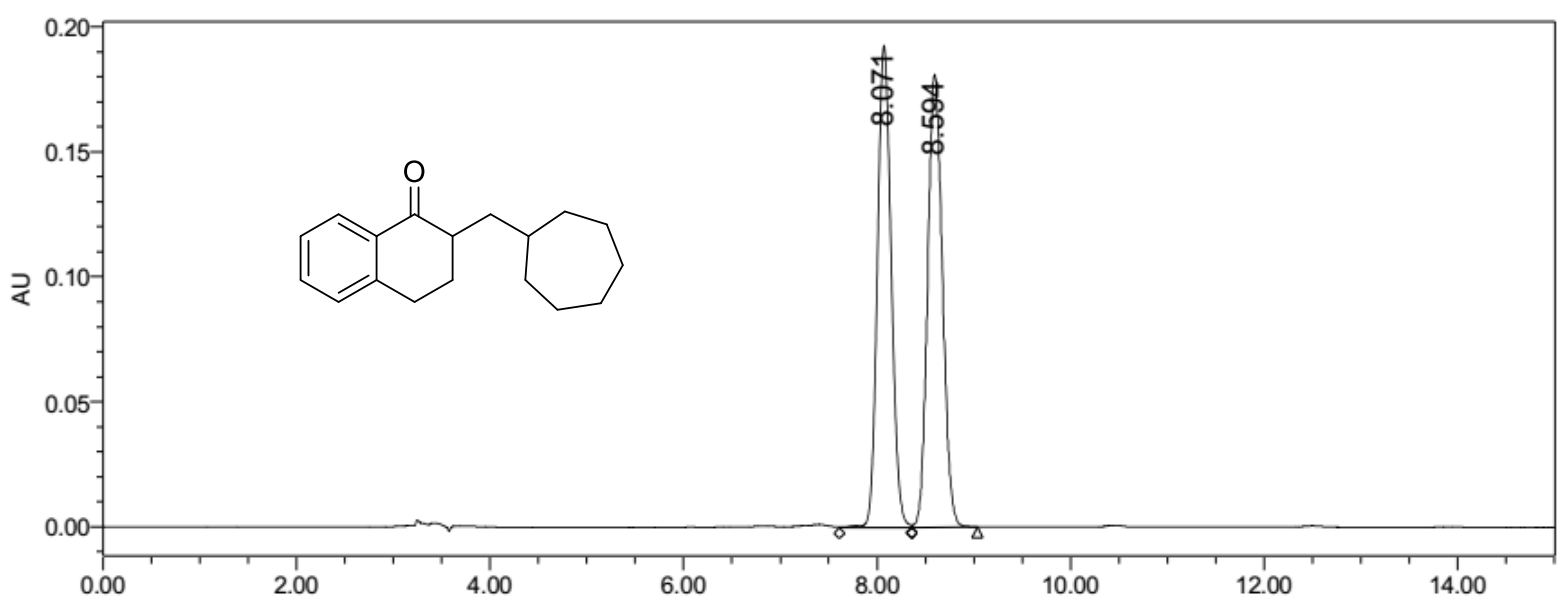

\begin{tabular}{|c|c|c|r|c|}
\hline & RT & Area & $\%$ Area & Height \\
\hline 1 & 8.071 & 1925078 & 50.17 & 192745 \\
\hline 2 & 8.594 & 1912046 & 49.83 & 181088 \\
\hline
\end{tabular}

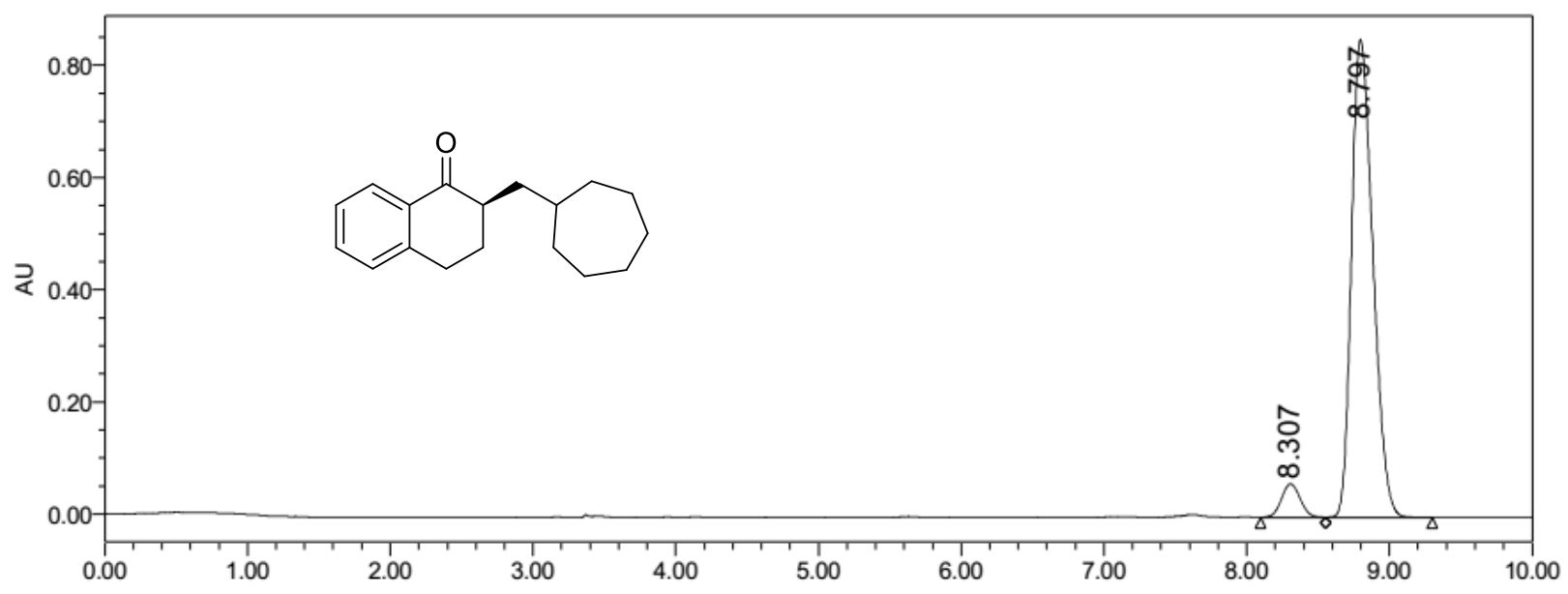

\begin{tabular}{|c|c|c|r|r|}
\hline & RT & Area & $\%$ Area & Height \\
\hline 1 & 8.307 & 542378 & 5.78 & 59740 \\
\hline 2 & 8.797 & 8848828 & 94.22 & 852193 \\
\hline
\end{tabular}




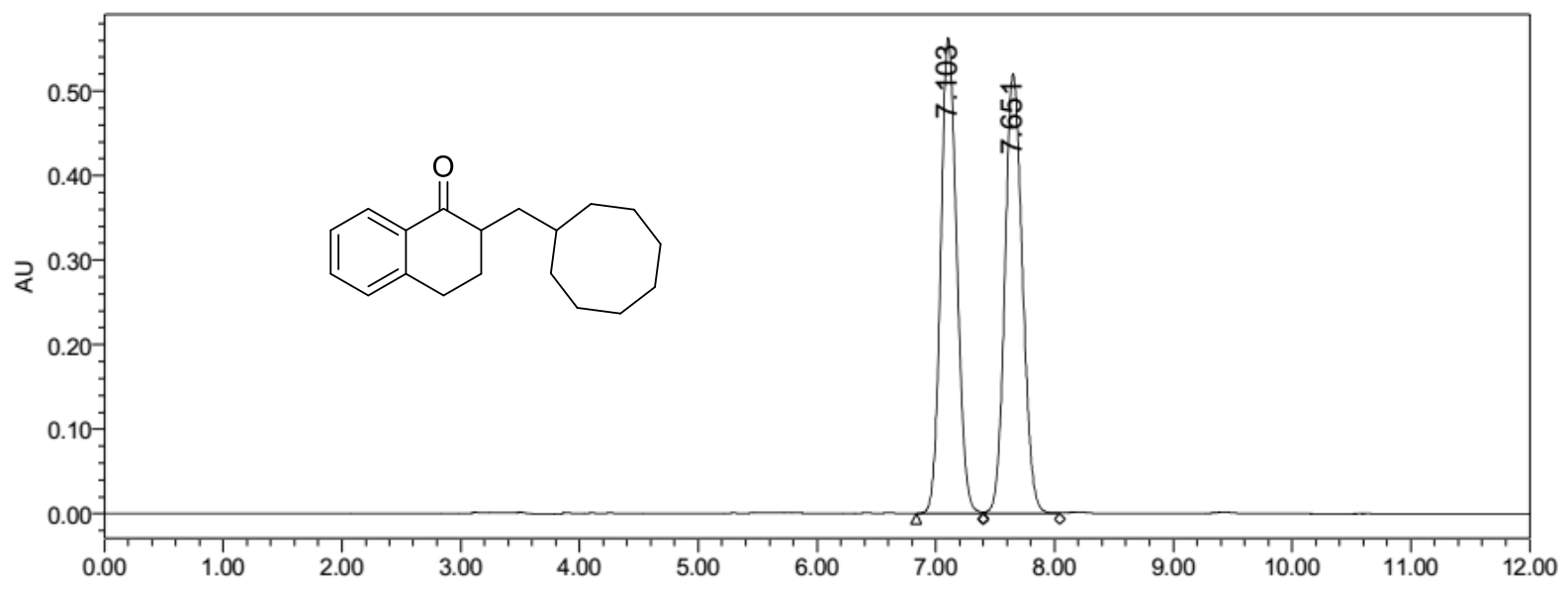

\begin{tabular}{|c|c|c|r|c|}
\hline & RT & Area & $\%$ Area & Height \\
\hline 1 & 7.103 & 5294465 & 49.88 & 562632 \\
\hline 2 & 7.651 & 5320946 & 50.12 & 519724 \\
\hline
\end{tabular}

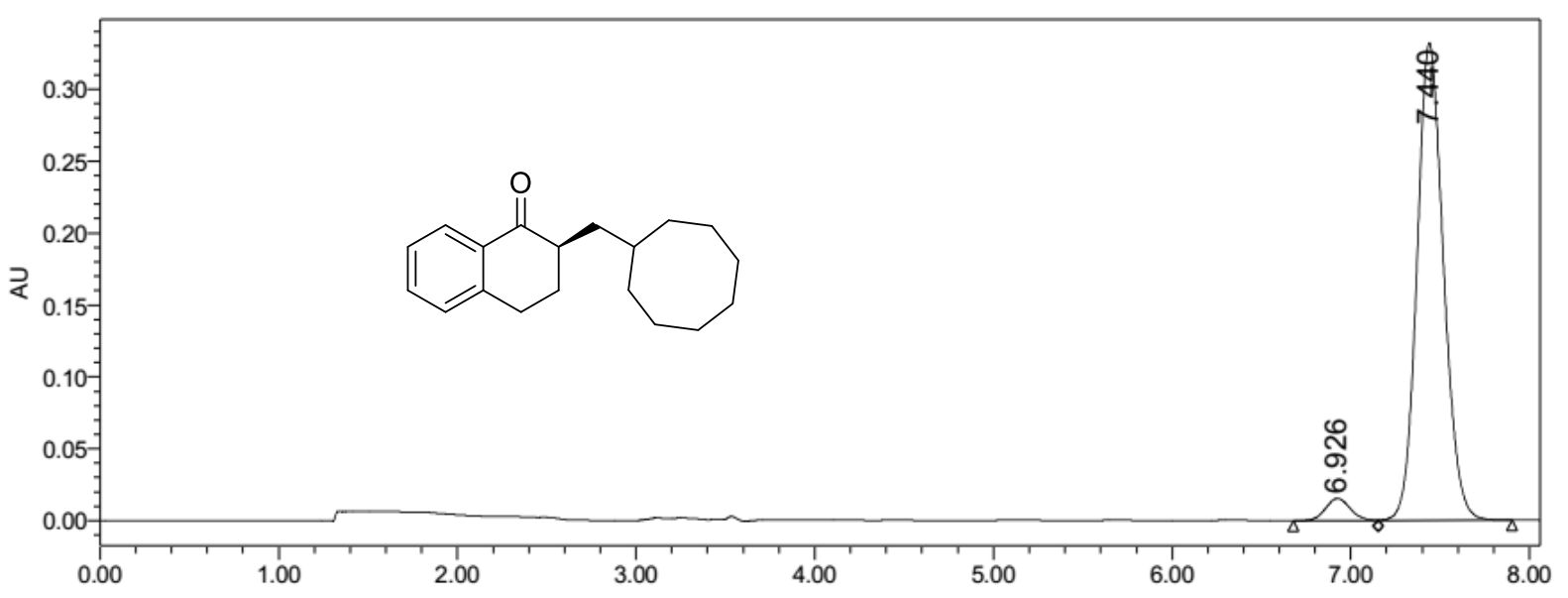

\begin{tabular}{|l|c|c|r|r|}
\hline & RT & Area & $\%$ Area & Height \\
\hline 1 & 6.926 & 147878 & 4.14 & 15418 \\
\hline 2 & 7.440 & 3420092 & 95.86 & 331786 \\
\hline
\end{tabular}



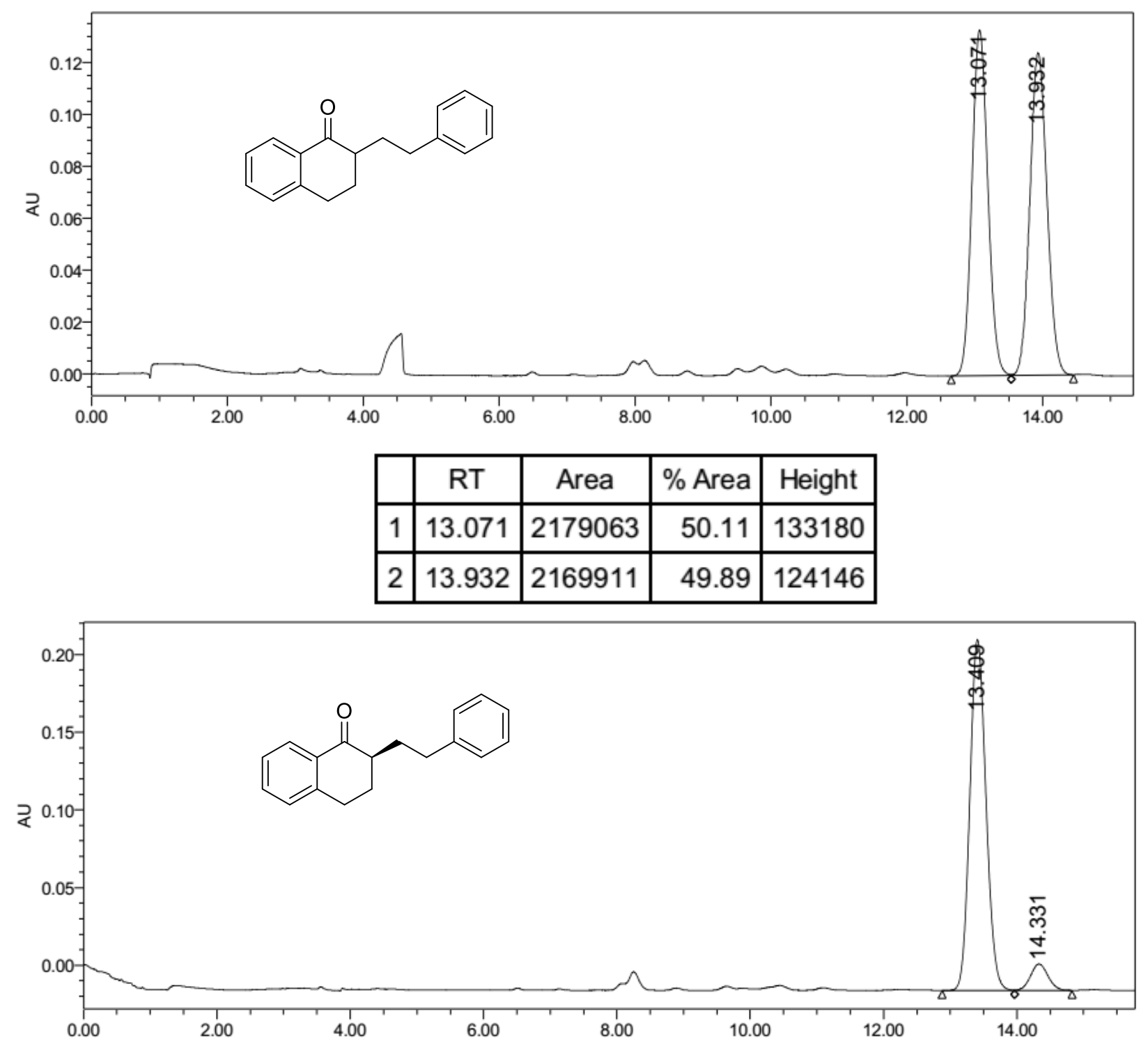

\begin{tabular}{|r|c|c|r|r|}
\hline & RT & Area & $\%$ Area & Height \\
\hline 1 & 13.409 & 3821776 & 92.48 & 225782 \\
\hline 2 & 14.331 & 310937 & 7.52 & 17002 \\
\hline
\end{tabular}




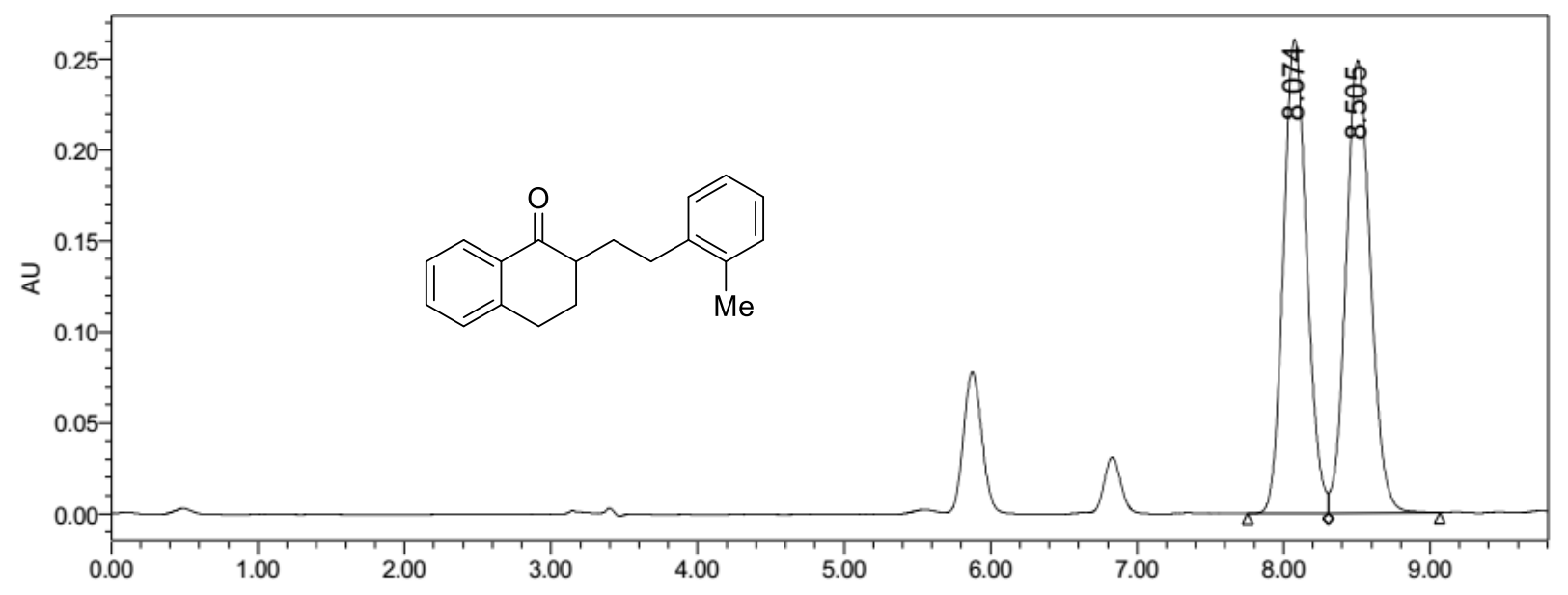

\begin{tabular}{|c|c|c|r|c|}
\hline & RT & Area & $\%$ Area & Height \\
\hline 1 & 8.074 & 2887928 & 49.82 & 260571 \\
\hline 2 & 8.505 & 2908432 & 50.18 & 248994 \\
\hline
\end{tabular}

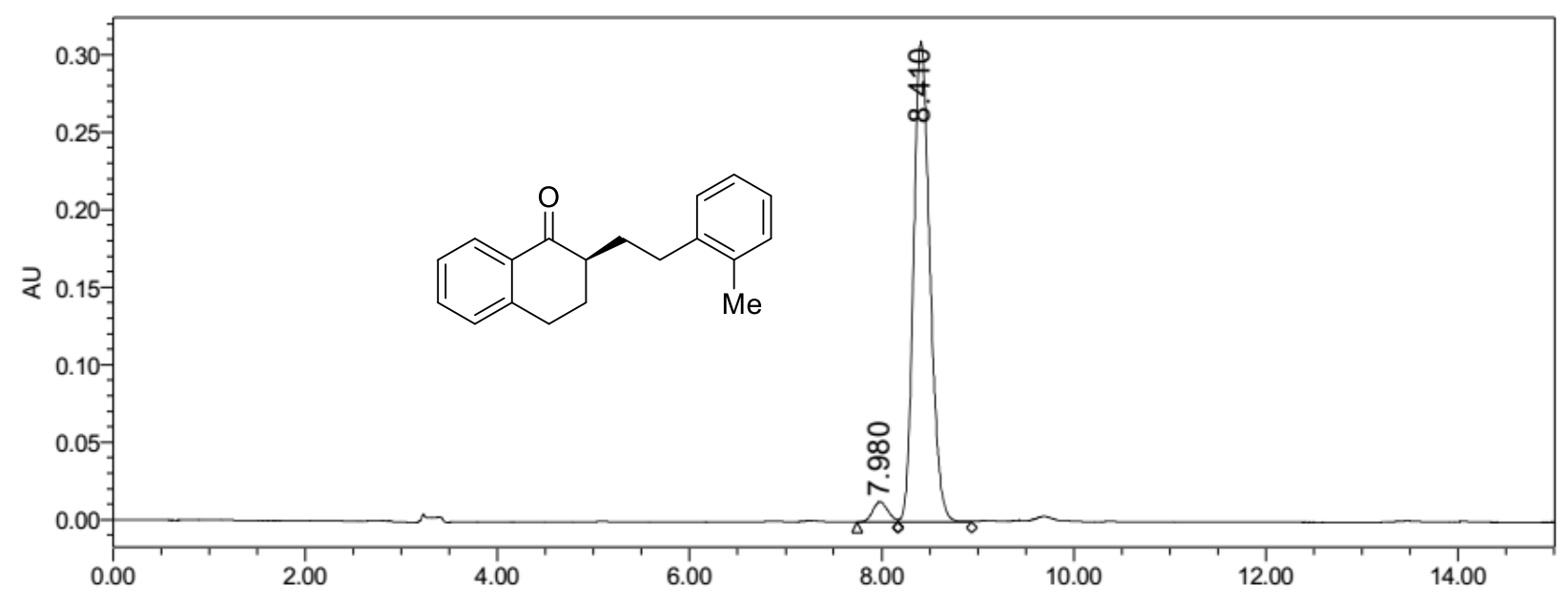

\begin{tabular}{|c|c|c|r|r|}
\hline & RT & Area & $\%$ Area & Height \\
\hline 1 & 7.980 & 142081 & 3.75 & 13062 \\
\hline 2 & 8.410 & 3651335 & 96.25 & 310221 \\
\hline
\end{tabular}




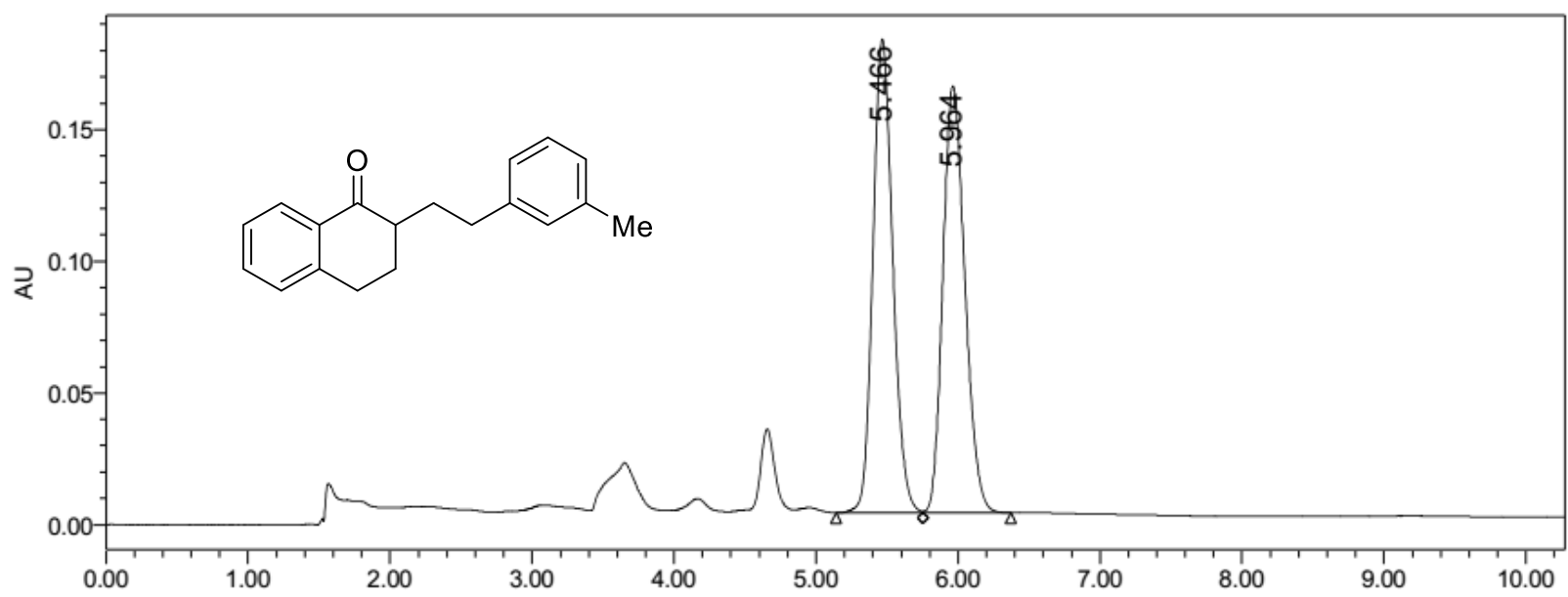

\begin{tabular}{|c|c|c|r|c|}
\hline & RT & Area & $\%$ Area & Height \\
\hline 1 & 5.466 & 1751868 & 50.21 & 179603 \\
\hline 2 & 5.964 & 1736889 & 49.79 & 161859 \\
\hline
\end{tabular}

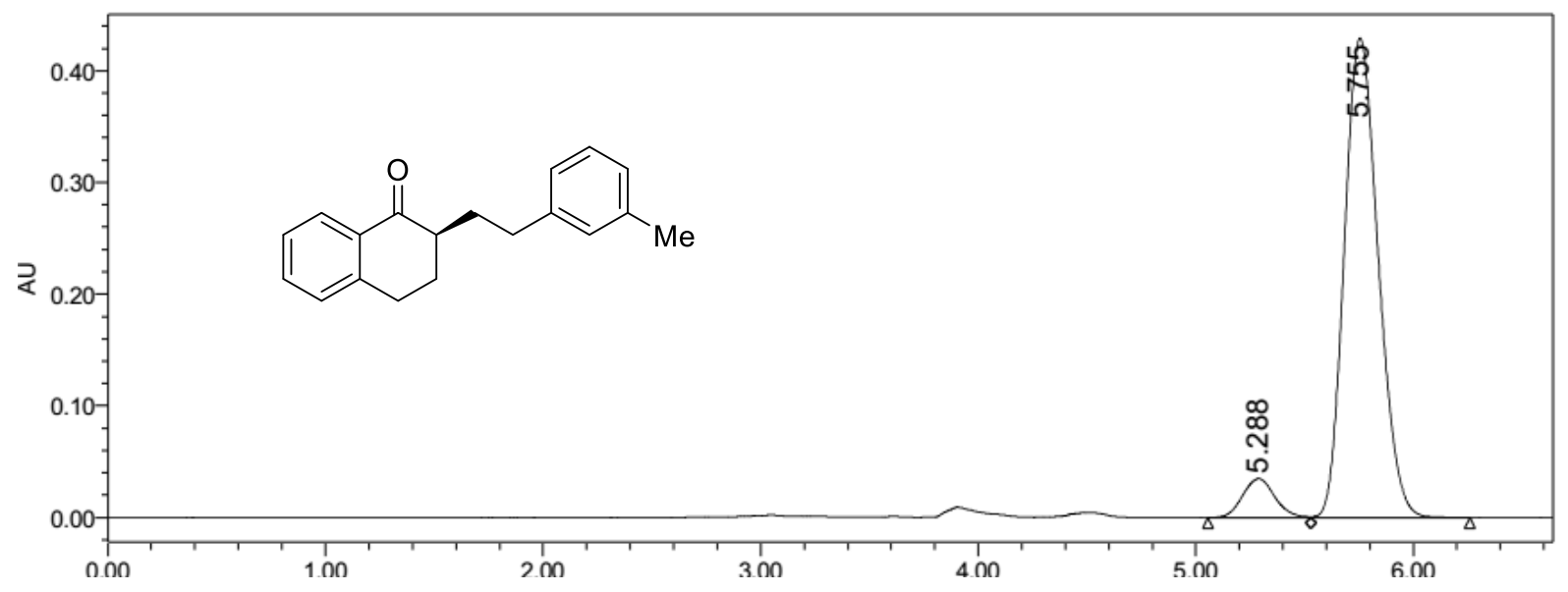

\begin{tabular}{|r|c|c|r|r|}
\hline & RT & Area & $\%$ Area & Height \\
\hline 1 & 5.288 & 358877 & 7.17 & 34884 \\
\hline 2 & 5.755 & 4648296 & 92.83 & 429218 \\
\hline
\end{tabular}




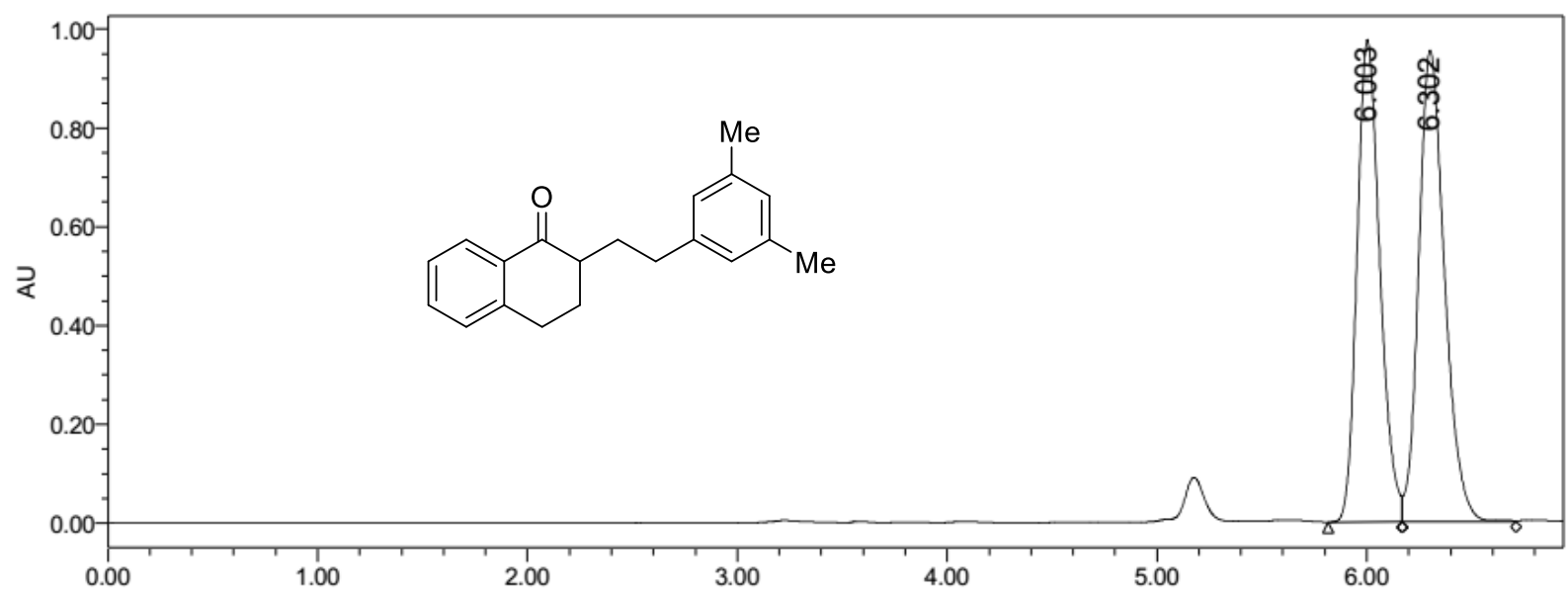

\begin{tabular}{|c|c|c|r|c|}
\hline & RT & Area & $\%$ Area & Height \\
\hline 1 & 6.003 & 7404728 & 47.54 & 976202 \\
\hline 2 & 6.302 & 8169647 & 52.46 & 953948 \\
\hline
\end{tabular}

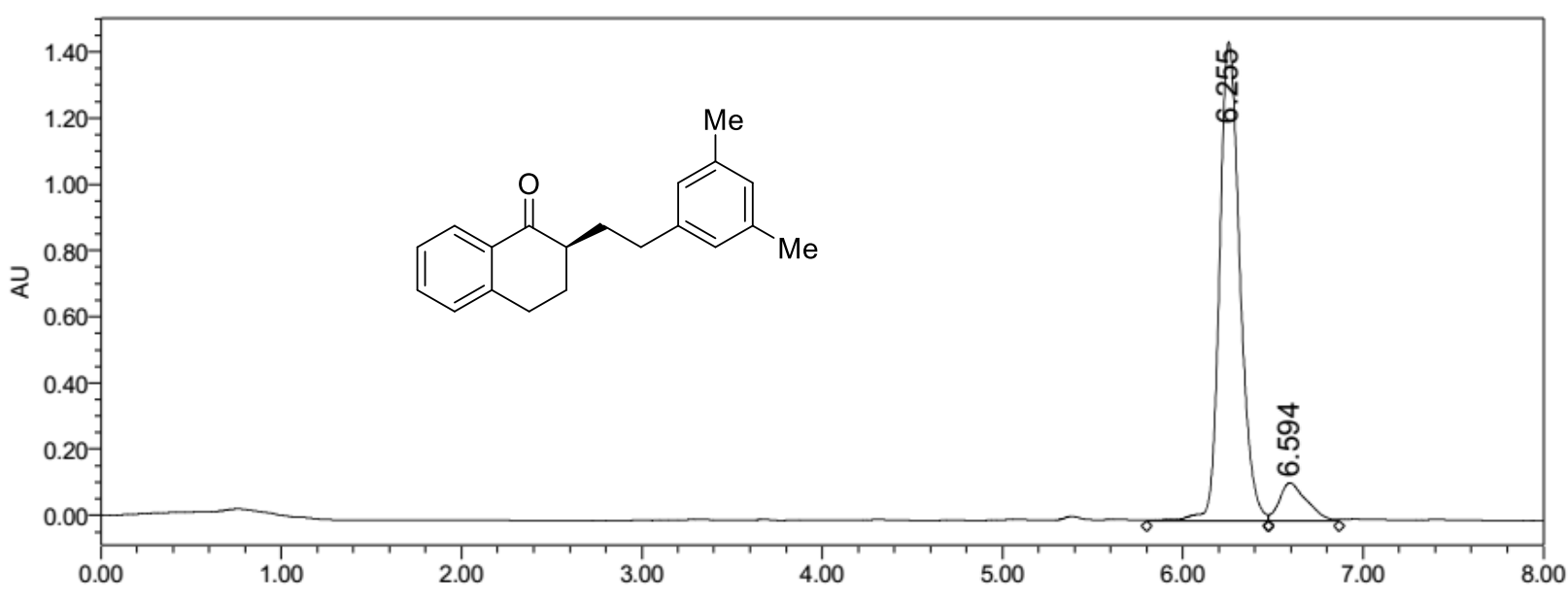

\begin{tabular}{|c|c|c|r|c|}
\hline & RT & Area & $\%$ Area & Height \\
\hline 1 & 6.255 & 11343619 & 90.51 & 1445962 \\
\hline 2 & 6.594 & 1188694 & 9.49 & 114315 \\
\hline
\end{tabular}




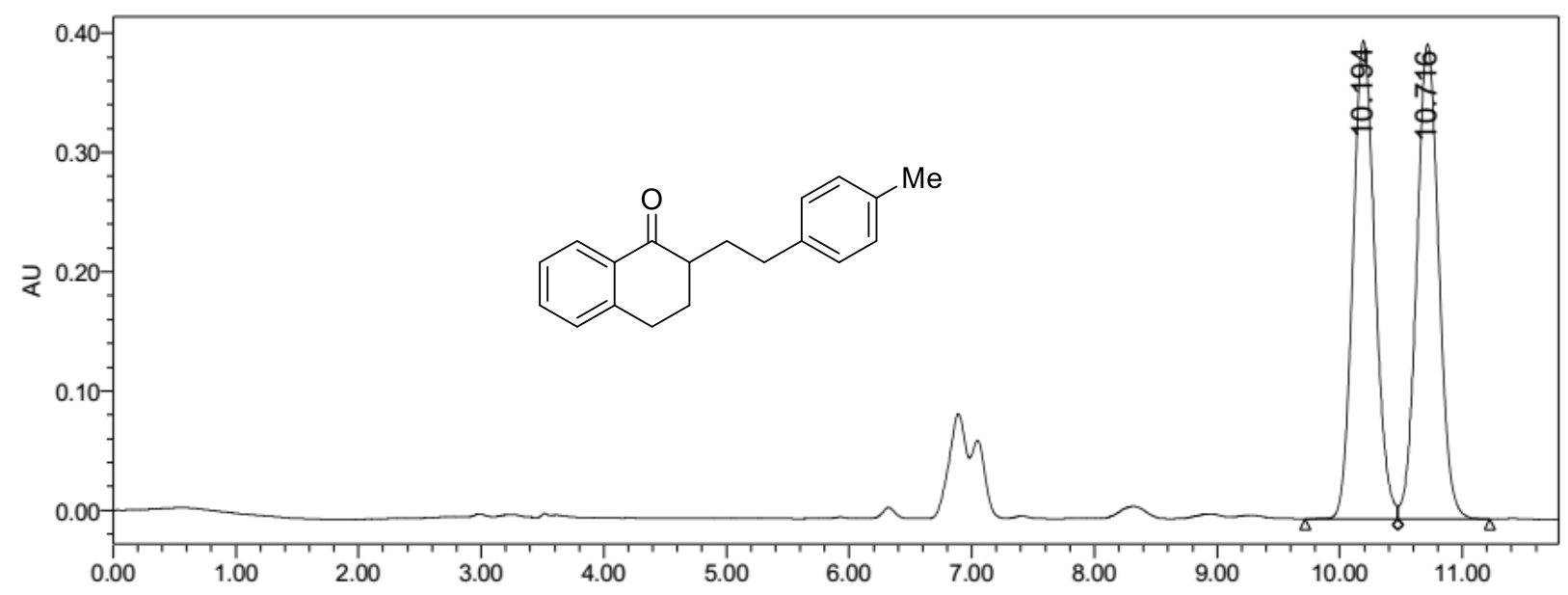

\begin{tabular}{|c|c|c|r|c|}
\hline & RT & Area & $\%$ Area & Height \\
\hline 1 & 10.194 & 4979117 & 50.12 & 401221 \\
\hline 2 & 10.716 & 4955572 & 49.88 & 398206 \\
\hline
\end{tabular}

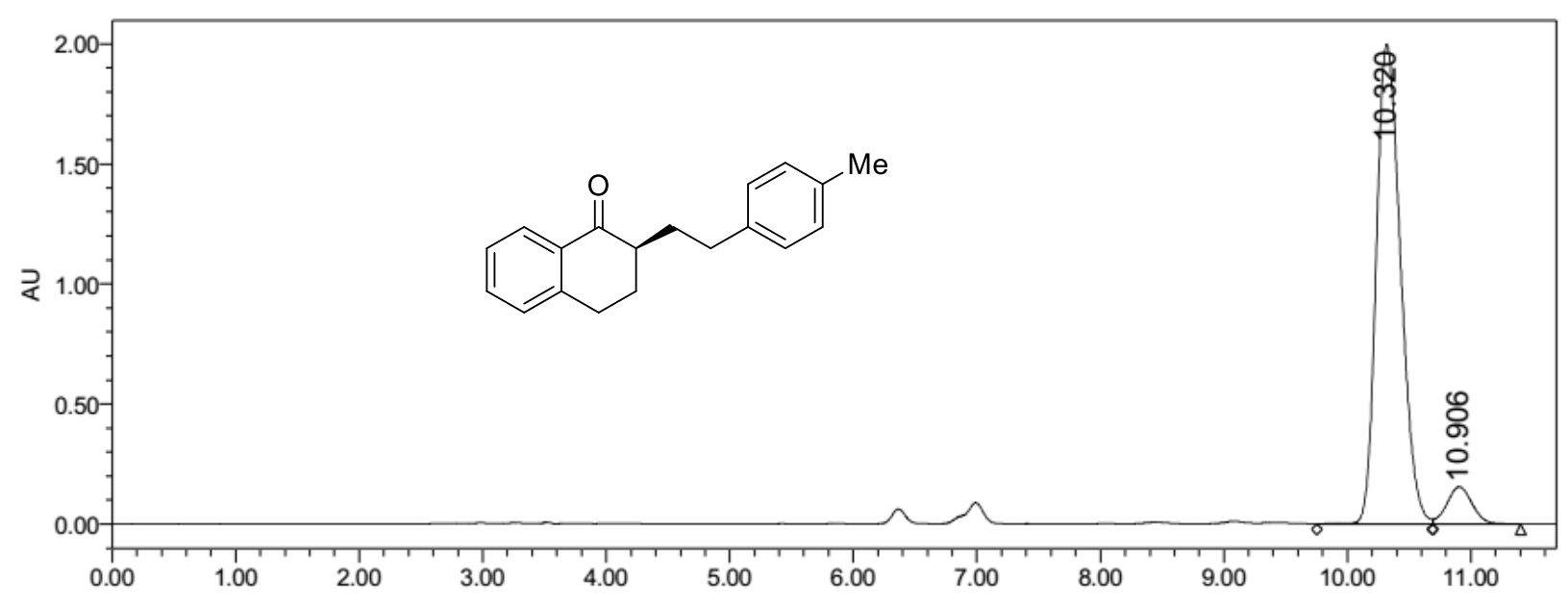

\begin{tabular}{|l|c|r|r|r|}
\hline & RT & Area & \% Area & Height \\
\hline 1 & 10.320 & 27103530 & 92.56 & 1997008 \\
\hline 2 & 10.906 & 2177068 & 7.44 & 154345 \\
\hline
\end{tabular}




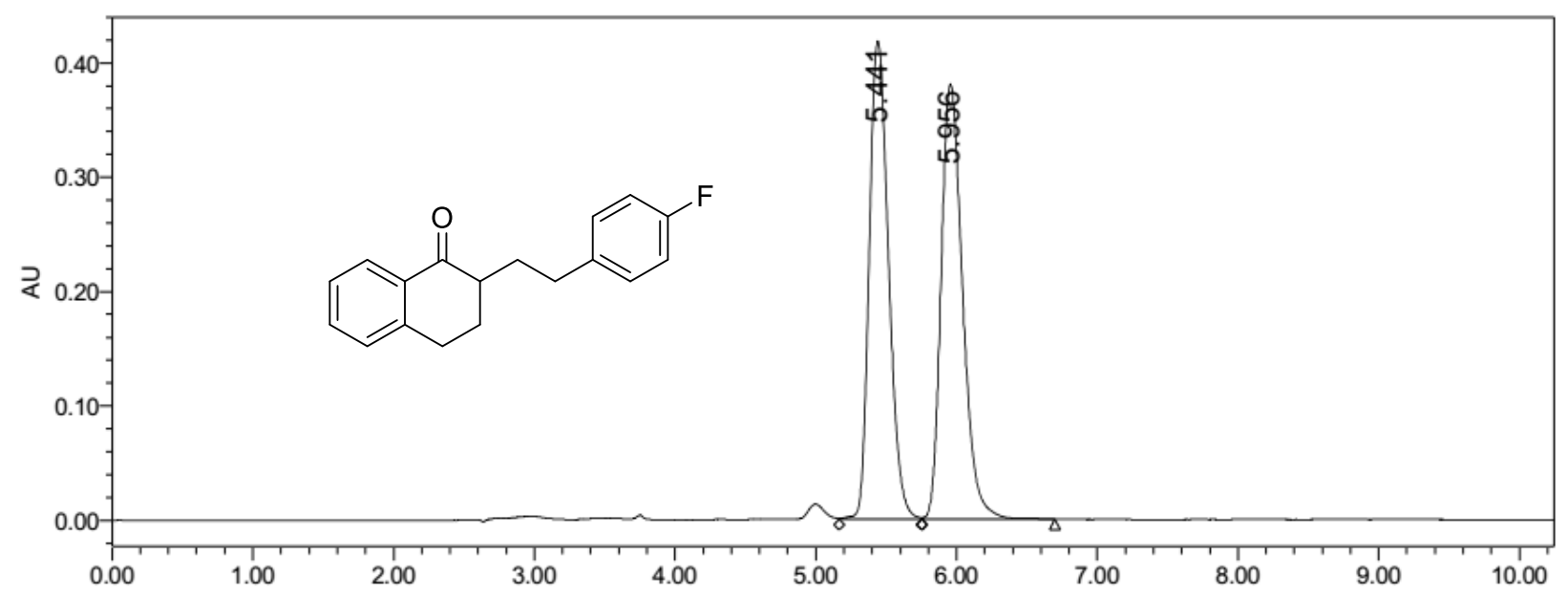

\begin{tabular}{|c|c|c|r|c|}
\hline & RT & Area & $\%$ Area & Height \\
\hline 1 & 5.441 & 4004829 & 49.84 & 418305 \\
\hline 2 & 5.956 & 4029992 & 50.16 & 380681 \\
\hline
\end{tabular}

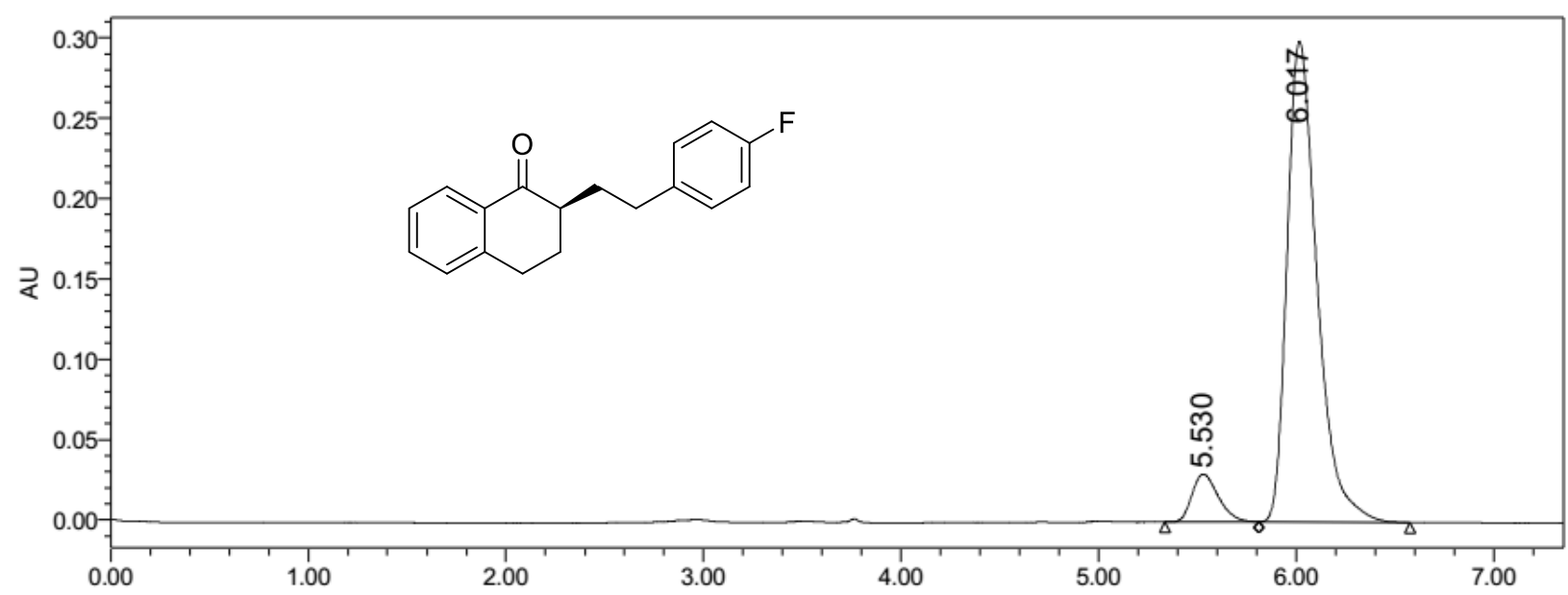

\begin{tabular}{|l|c|c|r|r|}
\hline & RT & Area & $\%$ Area & Height \\
\hline 1 & 5.530 & 277816 & 8.07 & 29788 \\
\hline 2 & 6.017 & 3163726 & 91.93 & 299196 \\
\hline
\end{tabular}




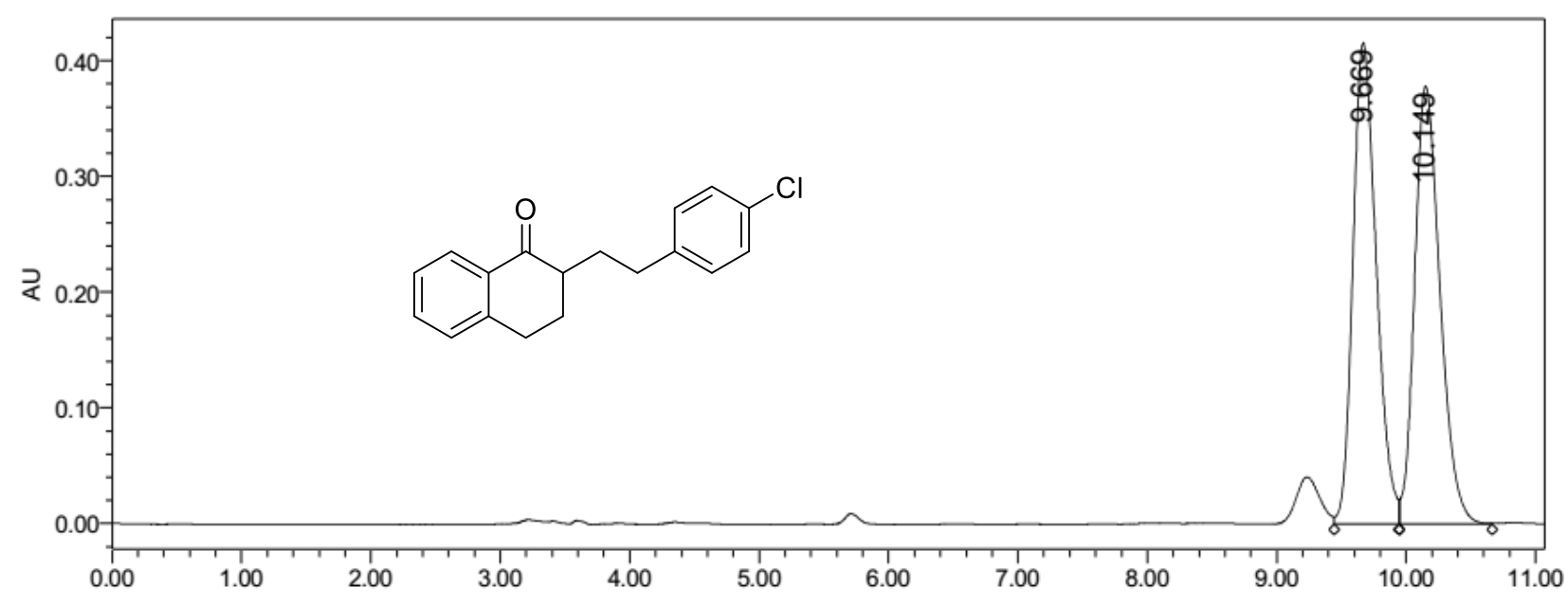

\begin{tabular}{|c|c|c|r|c|}
\hline & \multicolumn{1}{|c|}{ RT } & Area & $\%$ Area & Height \\
\hline 1 & 9.669 & 5203886 & 50.08 & 416148 \\
\hline 2 & 10.149 & 5186884 & 49.92 & 378503 \\
\hline
\end{tabular}

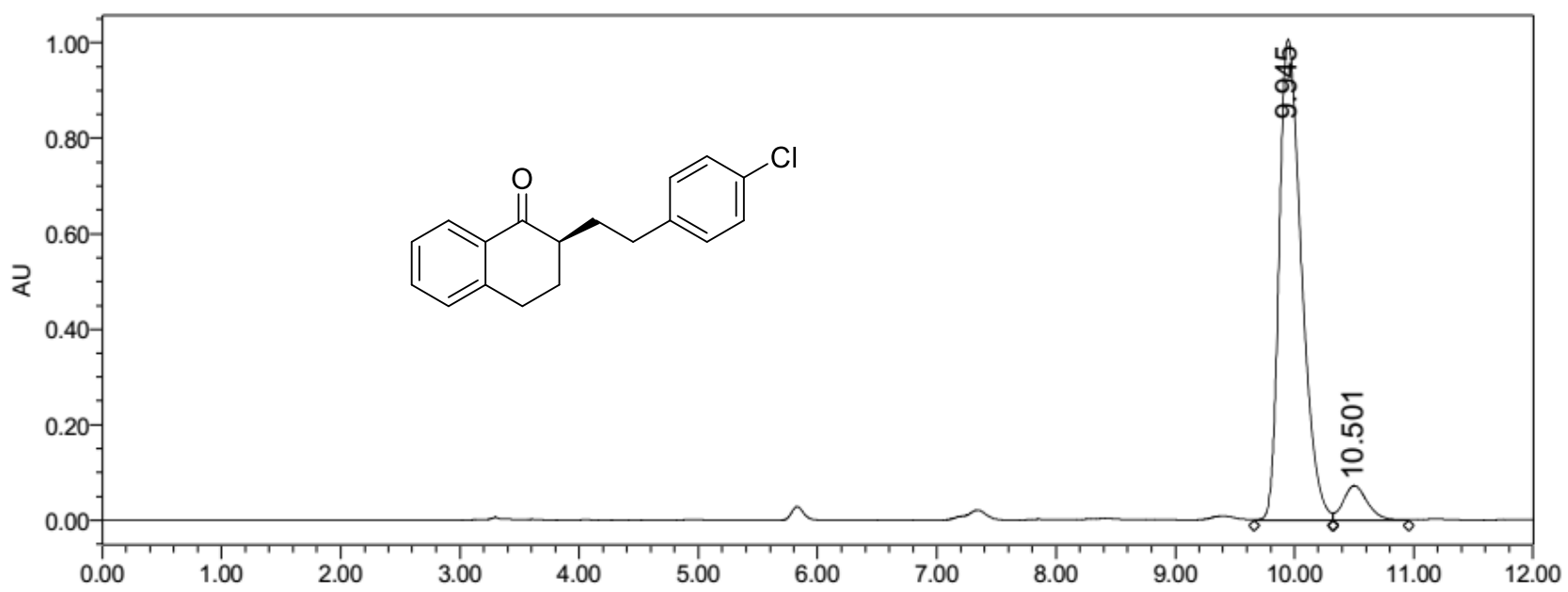

\begin{tabular}{|l|r|r|r|r|}
\hline & \multicolumn{1}{|c|}{ RT } & Area & $\%$ Area & Height \\
\hline 1 & 9.945 & 13097013 & 92.77 & 1007550 \\
\hline 2 & 10.501 & 1021256 & 7.23 & 72664 \\
\hline
\end{tabular}




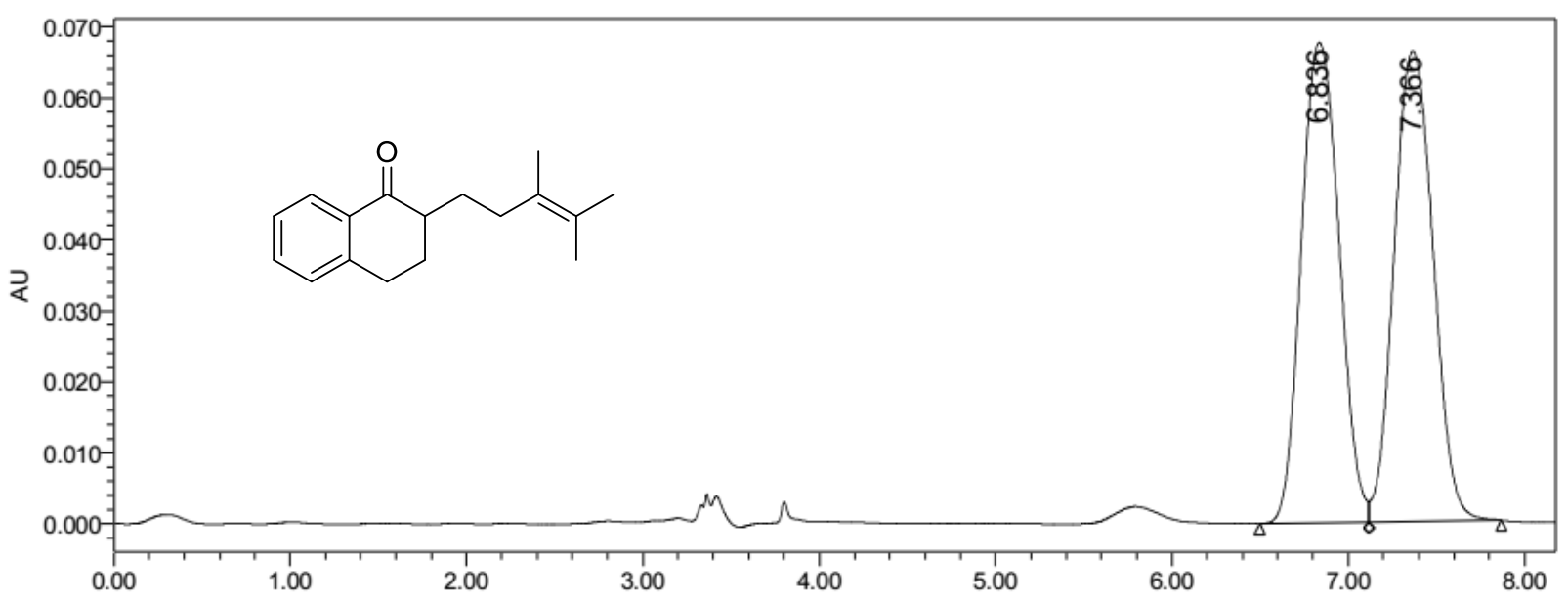

\begin{tabular}{|c|c|c|r|c|}
\hline & RT & Area & $\%$ Area & Height \\
\hline 1 & 6.836 & 1011809 & 49.78 & 67615 \\
\hline 2 & 7.366 & 1020945 & 50.22 & 66295 \\
\hline
\end{tabular}

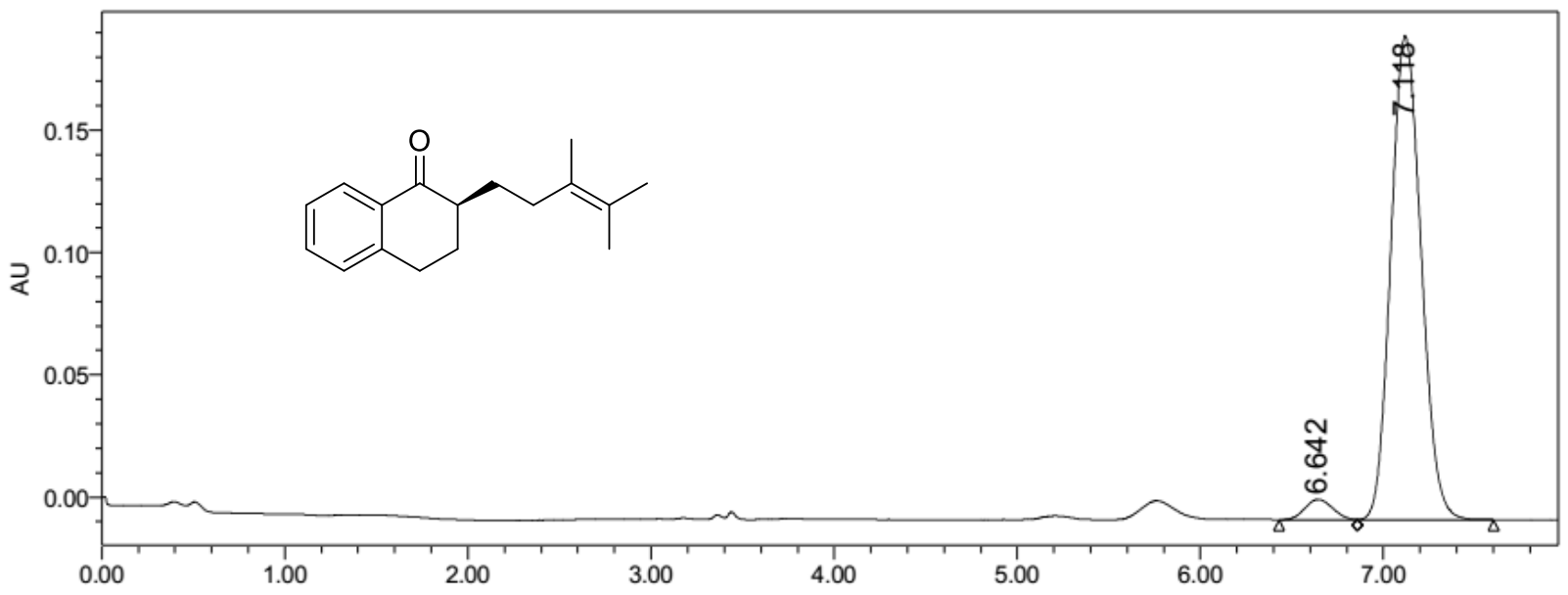

\begin{tabular}{|r|c|c|r|r|}
\hline & RT & Area & $\%$ Area & Height \\
\hline 1 & 6.642 & 87607 & 3.72 & 8319 \\
\hline 2 & 7.118 & 2268266 & 96.28 & 197816 \\
\hline
\end{tabular}




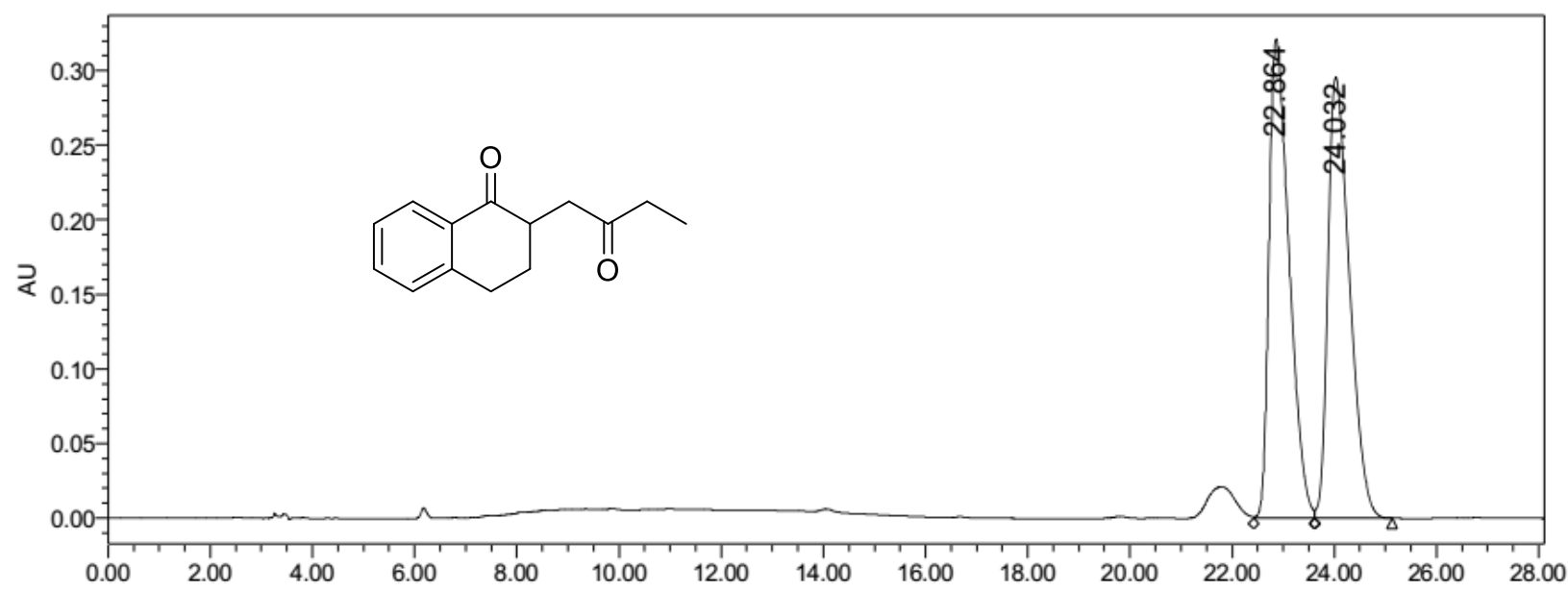

\begin{tabular}{|c|c|c|r|c|}
\hline & RT & Area & $\%$ Area & Height \\
\hline 1 & 22.864 & 8696853 & 49.92 & 321246 \\
\hline 2 & 24.032 & 8724048 & 50.08 & 295846 \\
\hline
\end{tabular}

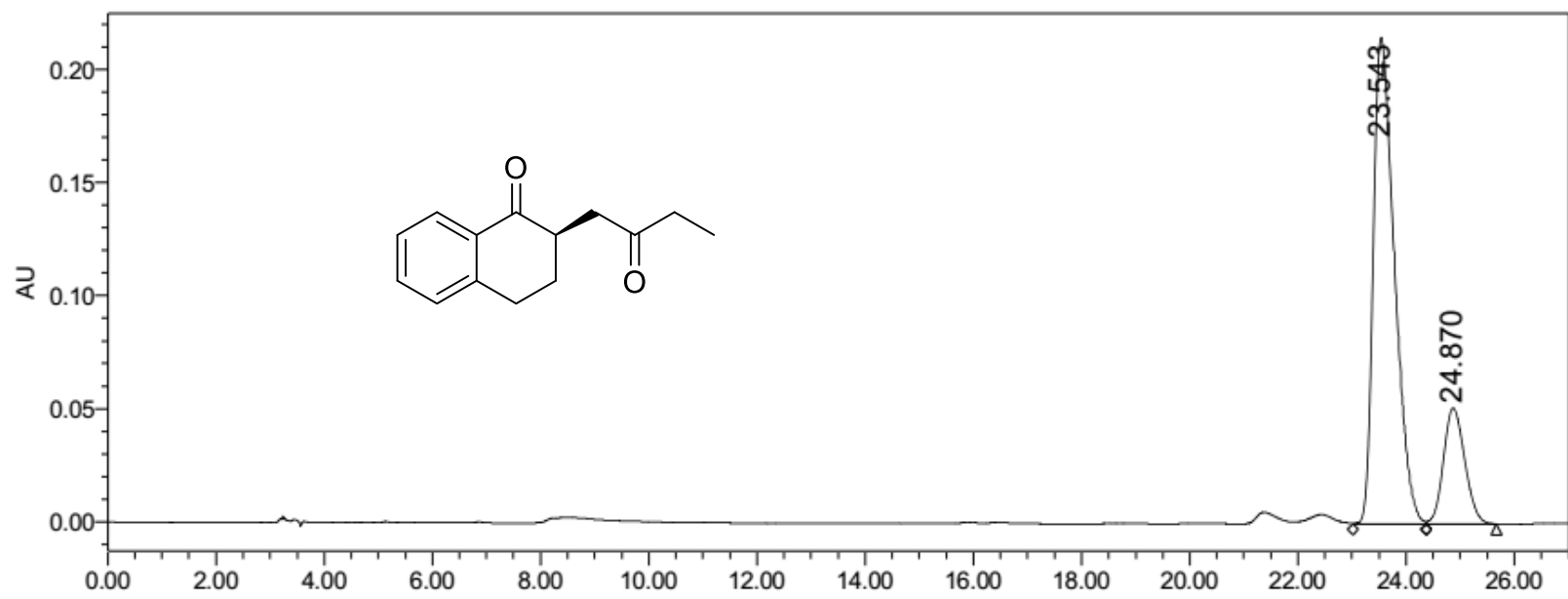

\begin{tabular}{|c|c|c|r|r|}
\hline & RT & Area & $\%$ Area & Height \\
\hline 1 & 23.543 & 5821282 & 80.52 & 214991 \\
\hline 2 & 24.870 & 1408137 & 19.48 & 51249 \\
\hline
\end{tabular}

